COMPRESSIVE MESOSCALE DAMAGE MODELING OF CONTINUOUS

FIBER-REINFORCED FLAX LAMINATES

\author{
by \\ Constantin Nicolinco \\ Bachelor of Engineering, Ryerson University (2015)
}

\begin{abstract}
A Thesis
presented to Ryerson University

in partial fulfillment of the

requirements for the degree of

Master of Applied Science

in the program of

Aerospace Engineering
\end{abstract}

Toronto, Ontario, Canada, 2017

(C) Constantin Nicolinco, 2017 


\section{AUTHOR'S DECLARATION FOR ELECTRONIC SUBMISSION OF A THESIS}

I hereby declare that I am the sole author of this thesis. This is a true copy of the thesis, including any required final revisions, as accepted by my examiners.

I authorize Ryerson University to lend this thesis to other institutions or individuals for the purpose of scholarly research.

I further authorize Ryerson University to reproduce this thesis by photocopying or by other means, in total or in part, at the request of other institutions or individuals for the purpose of scholarly research.

I understand that my thesis may be made electronically available to the public. 


\title{
COMPRESSIVE MESOSCALE DAMAGE MODELING OF CONTINUOUS FIBER-REINFORCED FLAX LAMINATES
}

\author{
Constantin Nicolinco \\ Master of Applied Science, Aerospace Engineering, Ryerson University, \\ Toronto (2017)
}

\begin{abstract}
Flax fibers have been observed to have specific mechanical properties on par with E-Glass. However, lack of knowledge on their mechanical behaviour as well as the absence of practical modeling tools have impeded the flax fiber from being used in structural applications. In this thesis, compressive mechanical testing was performed Flax/Epoxy laminates in order to capture and quantify the flax composite's non-linear behaviour with emphasis on damage and plasticity evolutions. A continuum damage mechanics-based on the standard Mesoscale Damage Theory (MDM) developed previously by Ladeveze and LeDantec was developed to include compressive damage and plasticity evolutions. The model parameters were derived from experimental data and optimized using open-source algorithms. Validations have been performed on Flax/Epoxy and EGlass/Polyester laminate composites in compression, as well as E-glass/Epoxy in tension. The model successfully predicts the composite's mechanical behaviour, and offers a robust predictive tool capable of aiding engineers and designers in the development of load-bearing natural fiber composites.
\end{abstract}




\section{ACKNOWLEGEMENTS}

I would like to thank the numerous people who have aided me towards the completion of this thesis, only some of whom are mentioned here:

This work could not have been completed without the constant support, guidance, and encouragement of my supervisor Dr. Habiba Bougherara. I am grateful for the resource she provided me with, and the opportunities I had working in her laboratories. I am equally indebted to $\mathrm{PhD}$ candidate Zia Mahboob of Ryerson University, who has been aiding my research from its infancy, and provided unmatched technical knowledge on the subjects.

I would also like thank Dr. Yves Chemisky and his research team from Arts et Métiers ParisTech, France, for implementing the Modified Mesoscale Damage Model into a multi-ply model, and for the patience he showed during my software learning curve.

I am most appreciative to Mr. David Miller and Mr. Andrei Betlen from Ryerson University's Computer Science Department for their expertise and support in the field of software development, integration, and implementation.

Even though this paper is solely written by me, the research and results provided here are a cumulative result of the work and time spent by the people mentioned above. 'I am forever grateful to you all'.

A special gratitude is extended to Huntsman Corporation (Huntsman Advanced Materials,

The Woodlands, TX) for supplying the research team with high-performance epoxy resins and hardeners.

Lastly, I would like to thank my mother Constanta, sister Elena, and cat Tzuika. They have not only providing me with the necessities required for the completion of this thesis, but also with unconditional love, support, patience, and understanding.

This research was partially funded by NSERC-DG. 


\section{DEDICATION}

This is dedicated to my lovely and supporting family, the single aspect of my life which I would never wish to change. 


\section{TABLE OF CONTENTS}

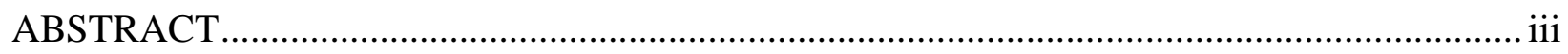

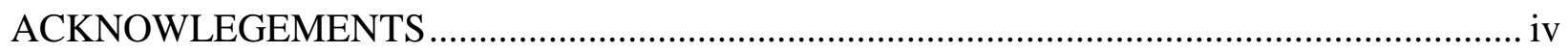

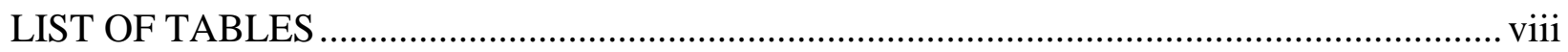

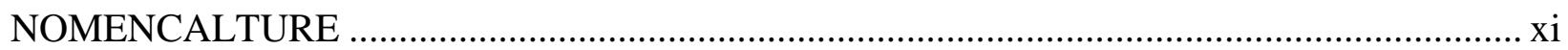

Chapter 1: Introduction and Background............................................................................. 1

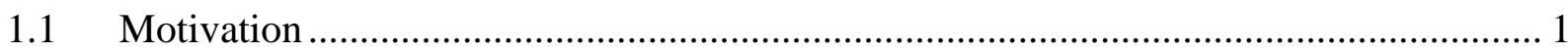

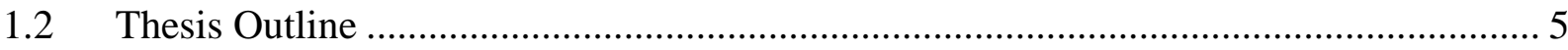

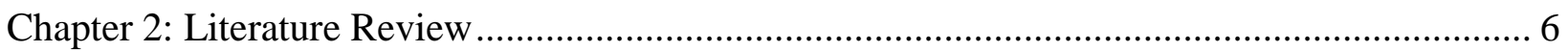

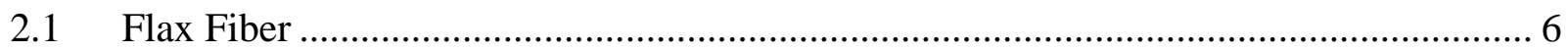

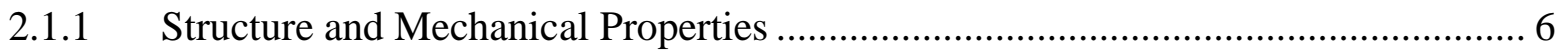

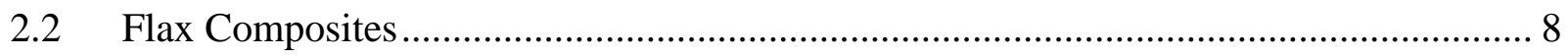

2.2.1 Damage Mechanics of Flax Composites in Compression ……….......................... 8

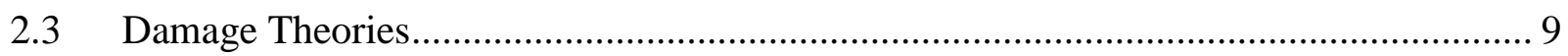

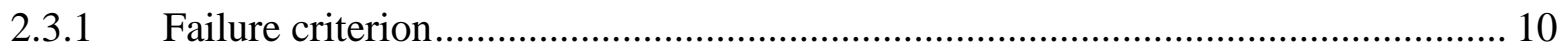

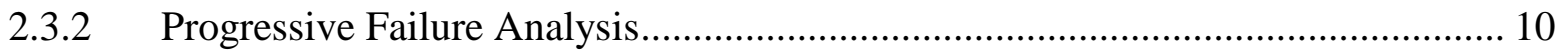

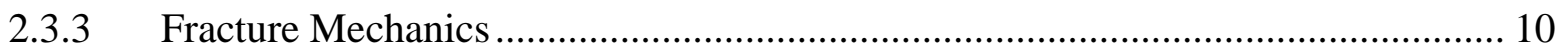

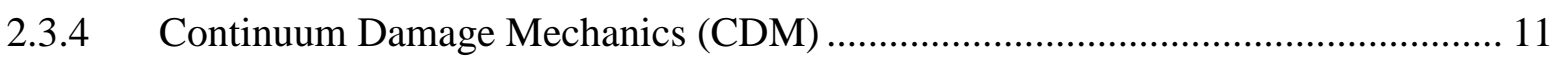

2.4 Standard Mesoscale Damage Theory (MDT) …………………................................ 11

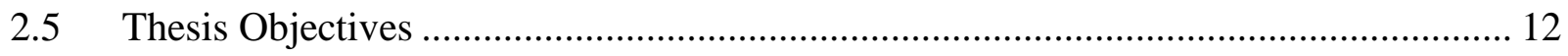

Chapter 3: Manufacturing, Quasi-Static Testing, and Observations ........................................... 17

3.1 Manufacturing Test Specimens ........................................................................ 17

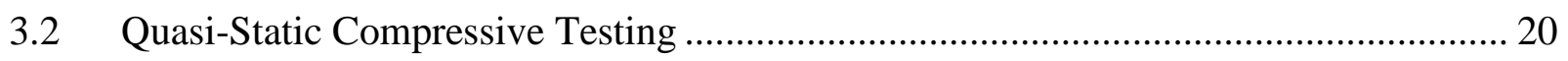

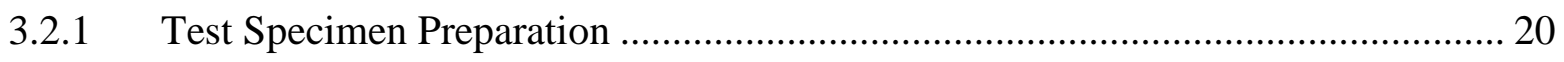

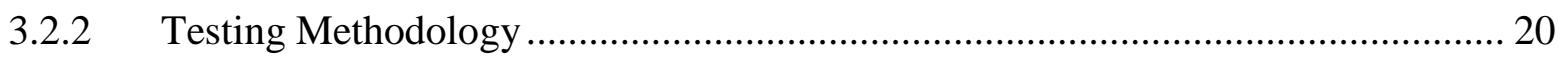

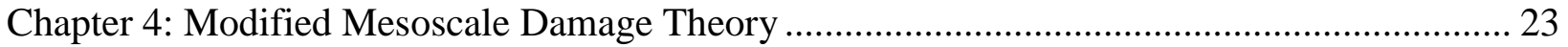

4.1 Modified Mesoscale Damage Theory ……………………………….................... 23

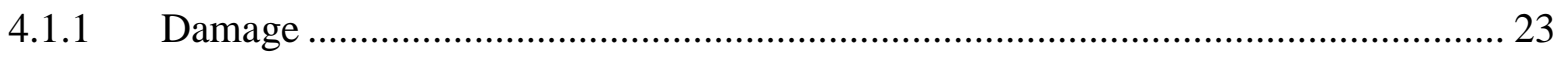

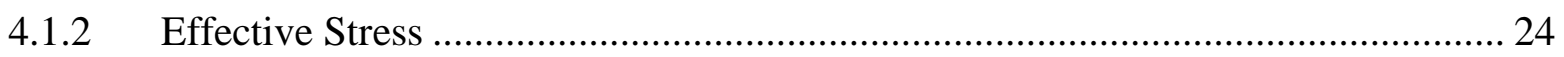

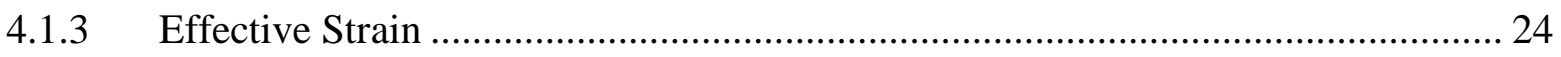

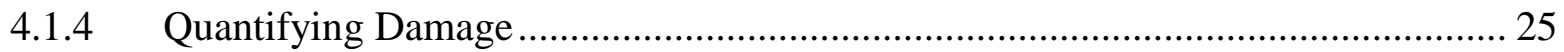

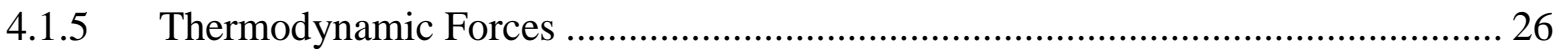




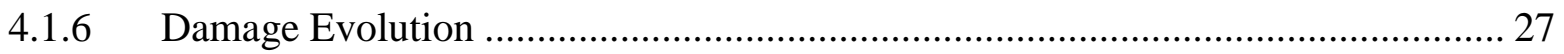

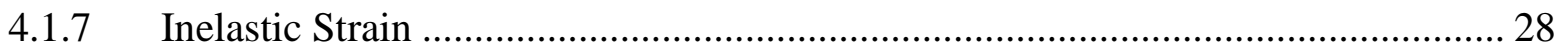

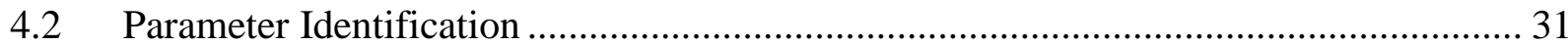

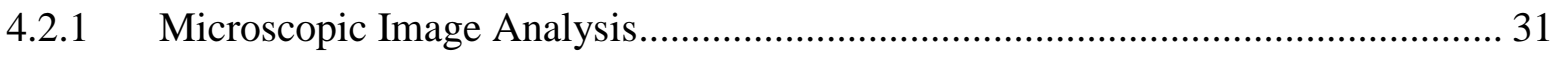

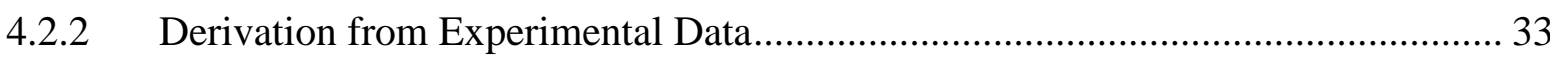

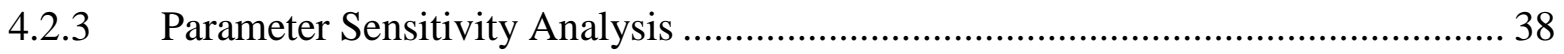

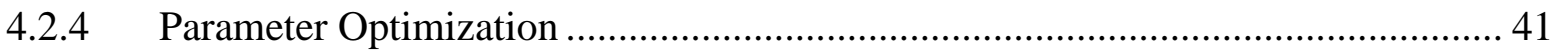

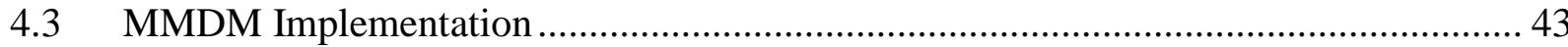

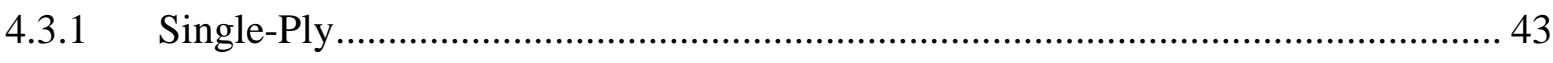

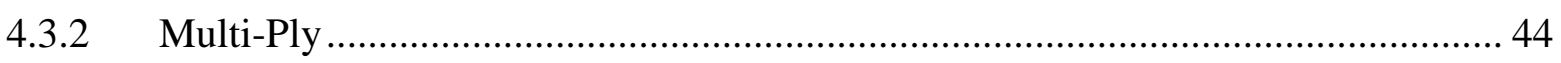

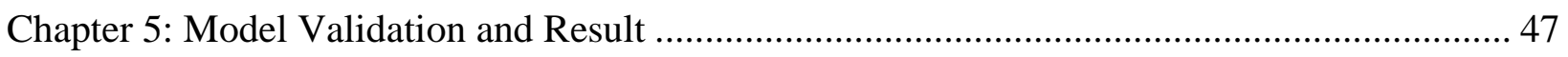

5.1 Model Validation on E-Glass Laminates …………………………………………...... 47

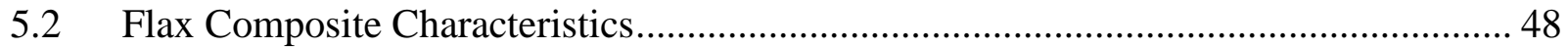

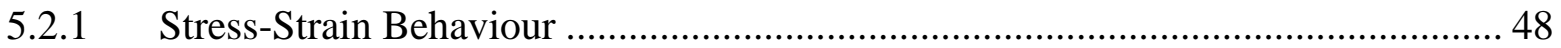

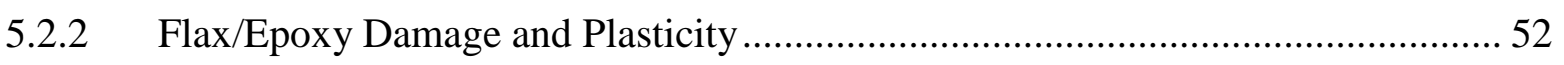

5.3 Flax/Epoxy Compressive Response and Simulation.................................................... 55

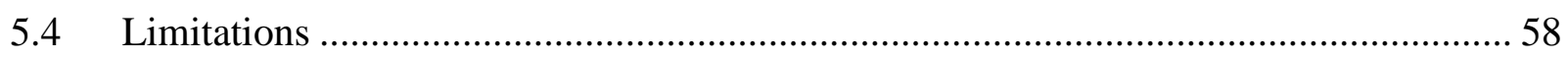

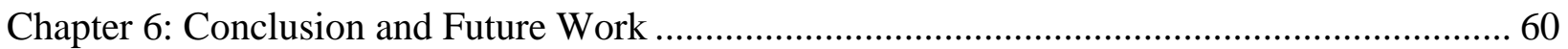

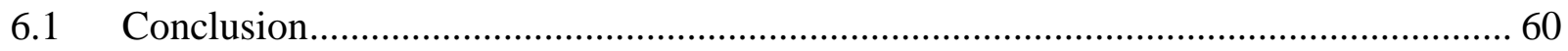

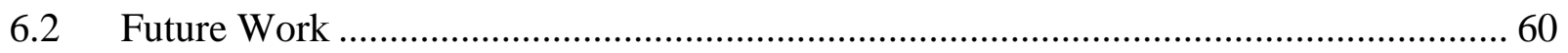

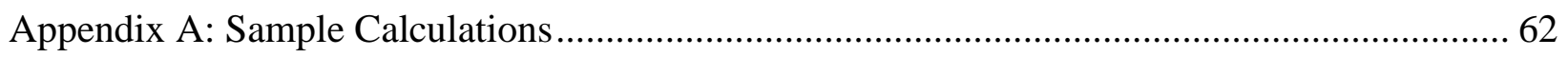

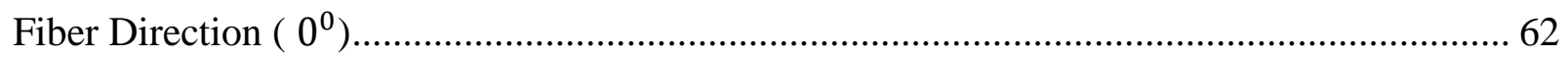

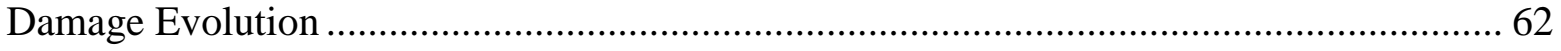

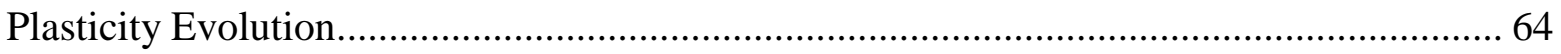

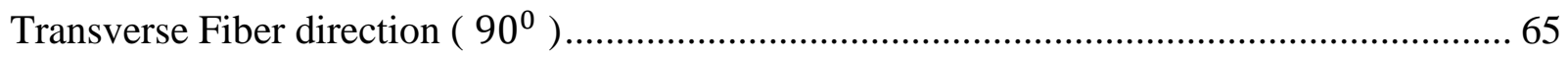

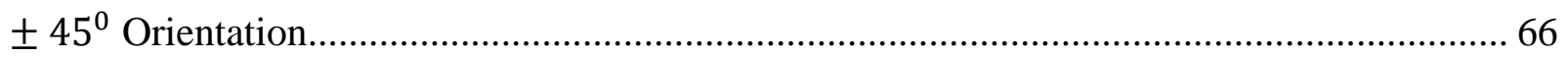

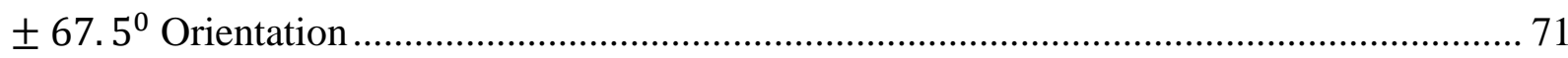

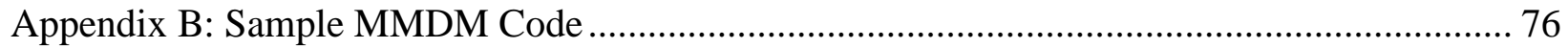

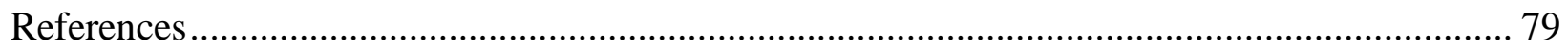




\section{LIST OF TABLES}

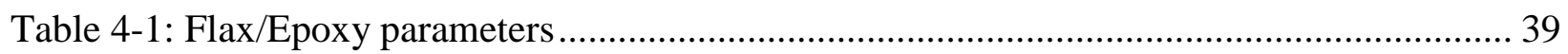

Table 5-1: Flax/Epoxy compressive-specific MMDM parameters ................................................ 56

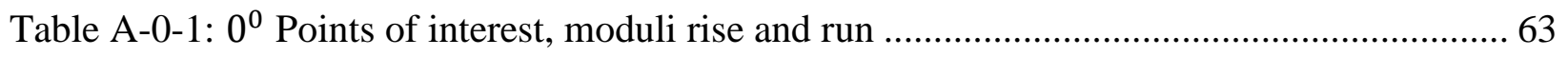

Table A-0-2: $0^{0}$ Elastic moduli, plastic strain, damage, and thermodynamic force....................... 64

Table A-0-3: $0^{0}$ Shear integrity, plastic strain, and yield function points ..................................... 65

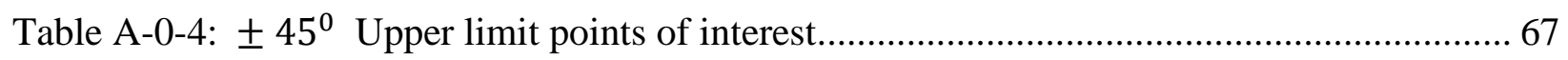

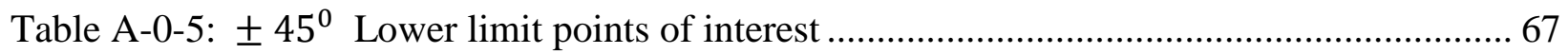

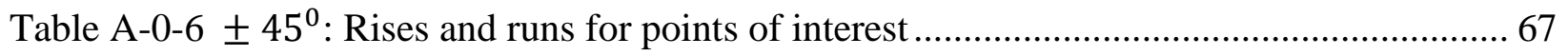

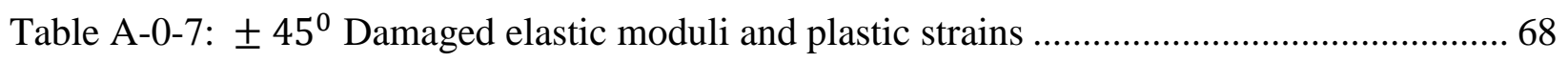

Table A-0-8: $\pm 45^{0}$ Calculated damages and thermodynamic force conjugates .......................... 68

Table A-0-9: $\pm 45^{0}$ Shear integrity, plastic strain, and yield function points ............................ 69

Table A-0-10: $\pm 45^{0}$ Points of interested and their respective rise and run................................ 69

Table A-0-11: $\pm 45^{0}$ Damaged elastic moduli, plastic strain, damage, and energy release rates 70

Table A-0-12: $\pm 45^{0}$ Shear integrity, plastic strain, and yield function points ............................. 71

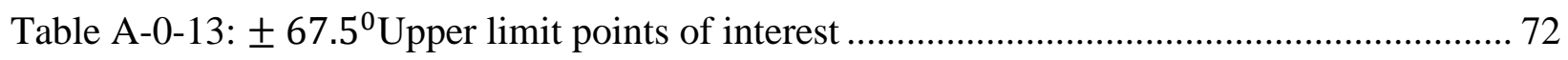

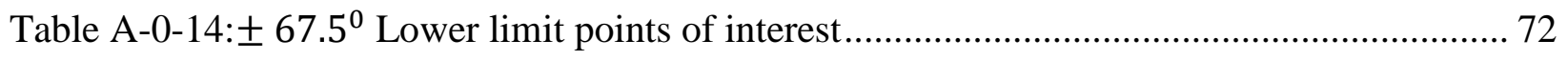

Table A-0-15: $\pm 67.5^{0}$ Rises and runs for points of interest................................................... 72

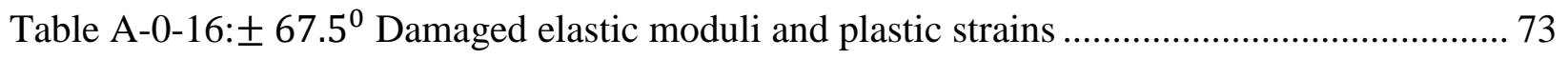

Table A-0-17: $\pm 67.5^{\circ}$ Calculated damages and thermodynamic force conjugates ....................... 73

Table A-0-18: $\pm 67.5^{0}$ Thermodynamic conjugates and damage initiation threshold.................. 74

Table A-0-19: $\pm 67.5^{0}$ Points of interested and their respective rise and run............................... 74

Table A-0-20: $\pm 67.5^{0}$ Damaged elastic moduli, plastic strain, damage, and energy release rates

Table A-0-21: \pm 67.5 Shear integrity, plastic strain, and yield function points.......................... 75 


\section{LIST OF FIGURES}

Figure 1-1: Environmental impacts from $1 \mathrm{~kg}$ fiber production, Flax vs Glass [8]..................... 2

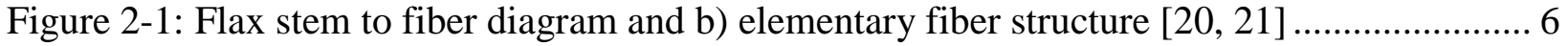

Figure 2-2: Flax fiber a) kink [22] b) transverse cracking initiating at a kink band [20] ............. 8

Figure 2-3: Failure modes of fibrous composite materials [32] ........................................... 9

Figure 3-1: Dry flax fiber used in specimen manufacturing ........................................... 17

Figure 3-2: a) Hot platen press stacking sequence; b) Carver Inc. self-containing press with heating/cooling platens and programmable controller......................................................... 19

Figure 3-3: Flax composite curing cycle ..................................................................... 19

Figure 3-4: Test specimen geometry a) composite and b) matrix-only ................................. 20

Figure 3-5: a) Testing procedure illustration, b) Test specimen with speckle pattern................ 21

Figure 3-6: Testing rig setup: a) Specimen in load cell b) Testing rig picture c) Testing rig

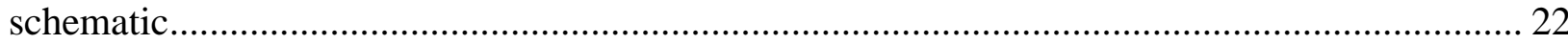

Figure 4-1: Damaged volume element...................................................................... 24

Figure 4-2: Damaged material inelastic response ........................................................ 29

Figure 4-3: a) Shear-transverse elastic domain function, b) power-law shaped shear-transverse

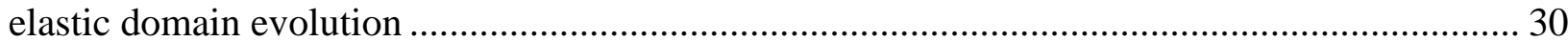

Figure 4-4: Grayscale SEM and binarized SEM .......................................................... 32

Figure 4-5: Grayscale SEM and binarized/thresholded SEM for crack isolation ..................... 33

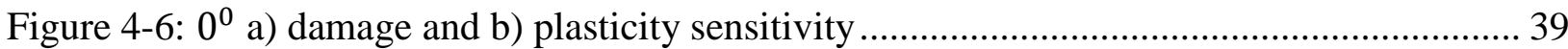

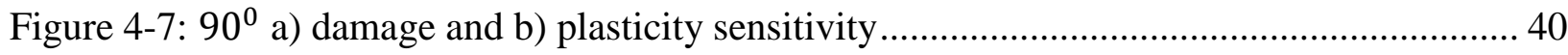

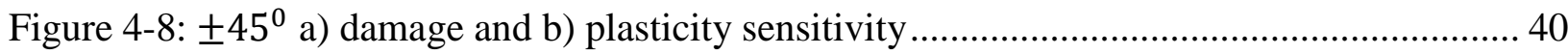

Figure 4-9: $\pm 67.5^{0}$ a) damage and b) plasticity sensitivity ............................................... 41

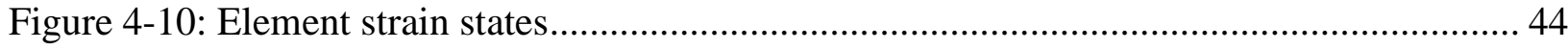

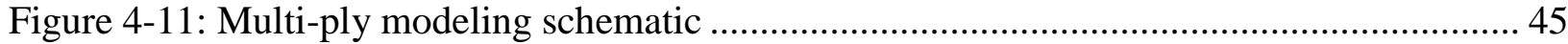

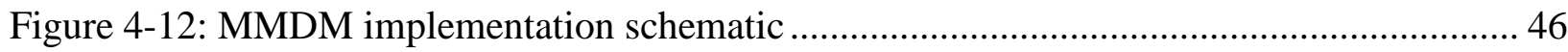

Figure 5-1: E-glass/Polyester composite compressive MMDM prediction vs experimental

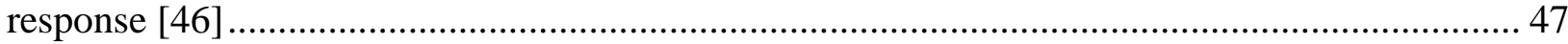

Figure 5-2: E-glass/Epoxy composite tensile MMDM prediction vs experimental response [46]48 Figure 5-3: Tensile and compressive UD Flax/Epoxy mechanical response from experimental data 
Figure 5-4: Tensile [29] and compressive cross-ply Flax/Epoxy mechanical response from

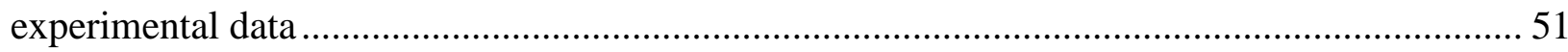

Figure 5-5: Fiber-direction a) Damage and b) Plasticity evolution ......................................... 52

Figure 5-6: Compressive shear-direction damage evolution: a) $\pm 45^{0}$, b) $\pm 67.5^{0} \ldots \ldots \ldots \ldots \ldots \ldots . . . . . . . . .53$

Figure 5-7: Compressive shear-direction plasticity evolution: a) $\pm 45^{0}$, b) $\pm 67.5^{0} \ldots \ldots \ldots \ldots \ldots \ldots . . . . . . .53$

Figure 5-8: Transverse exponential a) damage evolution, b) plasticity evolution...................... 54

Figure 5-9: Transverse a) linear damage evolution, b) gradual power plasticity evolution ......... 54

Figure 5-10: $0^{0}$ fiber-oriented Flax/Epoxy experimental behaviour and MMDM prediction...... 56

Figure 5-11: $\pm 67.5^{0}$ fiber-oriented Flax/Epoxy experimental behaviour and MMDM prediction 57

Figure 5-12: $90^{\circ}$ fiber-oriented Flax/Epoxy experimental behaviour and MMDM prediction ... 57

Figure 5-13: $\pm 45^{0}$ fiber-oriented Flax/Epoxy experimental behaviour and MMDM prediction 58 


\section{NOMENCALTURE}

\section{Abbreviations}

B.C.E. - Before Common Era

CMD - Continuum Damage Mechanics

DIC - Digital Image Correlation

FEA - Finite Element Analysis

MJ - Mega Joules

$\mathrm{MPa}$ - Mega Pascal

MDT - Mesoscale Damage Theory

MDM - Mesoscale Damage Model

MMDT - Modified Mesoscale Damage Theory

MMDM - Modified Mesoscale Damage Model

NFC - Natural Fiber Composite

SEM - Scanning Electron Microscopy

\section{Letters and Symbols}

$\mathrm{n}$ - specimen number

$\Omega-$ Shear integrity

$D$ - Damage

$\mathrm{S}$ - Area of a section volume element

$\sigma-$ Stress

$\varepsilon-$ Strain

$v$ - Poisson's ratio

$\tau-$ Shear stress

$\gamma$-Shear Strain

E-Elastic modulus

$E_{D}-$ Mean strain energy density

$\mathrm{G}$ - Shear modulus

$\rho$ - Density 
$p$ - Plastic strain

$\mathrm{Y}$ - Damage energy release rate

$\delta$ - Time derivative

b - Shear-transverse coupling constant

$\mathrm{t}$ - Time

$R(\tilde{p})$ - strain hardening function

$A_{t s}-$ Material coupling constant

B - Elastic properties and fiber orientation parameter

$\mathrm{k}$ - Elastic properties and fiber orientation parameter

$\alpha$ - Material parameter for the strain hardening curve

$\beta$ - Material parameter for the strain hardening curve

$\Phi$ - set of functions in regards to damage or plasticity evolution

$h_{i}\left(\tilde{p}_{i}\right)$ - hardening function dependent on the $i$ direction equivalent cumulative effective inelastic strain $\left(\tilde{p}_{i}\right)$

$\Delta$ - Difference or change of a certain quantity

\section{Subscripts}

11 - Fiber direction

22 - Transverse direction

12 - Shear direction

33 - Out of plane direction

$\mathrm{X}$ - Longitudinal axis direction

$\mathrm{Y}$ - Transverse axis direction

$X Y$ - In-plane shear direction

$f$ - Fiber direction

$s$ - Shear

$t$ or $\mathrm{T}-$ Transverse

$t s-$ Transverse- shear

L - Longitudinal

$i$ - referring to an $\mathrm{i}^{\text {th }}$ entry in a certain column or list 
$i j-$ referring to an entry in the $\mathrm{i}^{\text {th }}$ row and $\mathrm{j}^{\text {th }}$ column of a matrix

LL - Lower limit

UL - Upper limit

$\mathrm{r}-$ referring to the $\mathrm{r}^{\text {th }}$ ply

\section{Superscripts}

$\dot{a}$ - Time derivative of $a$

$\vec{a}$ - Updated value of $a$

$\sim-$ Effective

E or e - Elastic

D - Damaged

C - Critical

$y$ - Yield

0 - Initial or Undamaged

'- Smaller magnitude

“ - Larger magnitude

u - Ultimate

U - Upper

L - Lower

p-Plastic

$y$ - Yield

eq-Equivalent

$\mathrm{t}$ - Tangent

$\mathrm{T}$ - Tensile 


\section{Chapter 1: Introduction and Background}

With a continuously increasing demand for materials with high specific strengths and low weight, continuous fiber-reinforced composites have become a popular choice in major industries such as automotive, aerospace, and marine engineering. Carbon and Glass fibers are the most popular and versatile continuous-fibers used for composite reinforcement [1-3]. Their use in industry has been enjoying a continuously steady growth. It is projected that these fibers will continue prospering at exponential rates for the foreseeable future [1, 2]. However, energyintensive manufacturing, non-renewability, and poor recyclability have created an interest in sustainable bio-based alternatives such as natural fibers with a lesser manufacturing cost and environmental impact. There are three main categories of natural fiber sources: 1) mineral (asbestos group), 2) animal (silk, wool, hair, etc), 3) plant (flax, hemp, jute, sisal, coconut, etc.). Considering the fact that mineral-based fibers can cause serious health conditions such Asbestosis and a large variety of cancers (esophagus, lung, chest, abdomen, kidney etc.) [4], they have to be avoided in mass-manufacturing operations involving any human contact. Animal fibers are generally neutral to human health, however their high price to mechanical performance ratio is unpractical for structural applications [4]. Therefore, only plant-based fibers currently offer a source of cost-effective and renewable fibers with sufficiently high mechanical properties for structural applications.

\subsection{Motivation}

There are several advantages and disadvantages associated with natural fibers composites (NFCs) compared to synthetic fibers, some of them are listed below [5, 6]:

\section{Advantages}

- Lower density

- Lower manufacturing cost

- Easier manufacturing

- Less lifecycle environmental damage

- Sustainable

- Good energy absorption properties

\section{Disadvantages}

- Lower ultimate strengths

- Inconsistent geometry and wide scatter of mechanical properties

- Prone to natural decomposition

- High water absorption

- Sensitive to operating temperatures 
Several plant fibers have been found to have specific mechanical properties comparable to Glass fibers, while having a significantly lower manufacturing cost per unit weight. From the works of Zsriros [7], flax has been identified to compare most favorably with E-glass in terms of cost and mechanical performance, while its manufacturing non-renewable energy consumption per unit weight is 4 times less (11.7 MJ/kg for flax and 45MJ/kg for E-glass [8]).

Table 1-1: Flax vs E-Glass performance and cost comparison $[4,5]$

\begin{tabular}{|c|c|c|c|c|c|}
\hline Material & $\begin{array}{c}\text { Specific Strength } \\
\left(\mathrm{MPa} / \mathrm{gcm}^{3}\right)\end{array}$ & $\begin{array}{c}\text { Specific Modulus } \\
\left(\mathrm{GPa} / \mathrm{gcm}^{3}\right)\end{array}$ & $\begin{array}{c}\text { Material Cost } \\
(\$ / \mathrm{lb})\end{array}$ & $\begin{array}{c}\text { Cost per ksi of } \\
\text { tensile strength } \\
(\$ / \mathrm{ksi})\end{array}$ & $\begin{array}{c}\text { Cost per ksi of } \\
\text { flexural } \\
\text { modulus }(\$ / \mathrm{ksi})\end{array}$ \\
\hline Flax & 1300 & $20-70$ & 0.60 & 0.15 & 0.003 \\
\hline E-glass & 1350 & 30 & 1.2 & 0.17 & 0.003 \\
\hline
\end{tabular}

Plant fibers are renewable, $\mathrm{CO}_{2}$ neutral, recyclable, and easier to process when compared to synthetic fibers [9, 10]. Figure 1-1 [8] presents a radial graph comparing the environmental impact per unit weight of flax fibers intended for composite applications versus glass fibers.

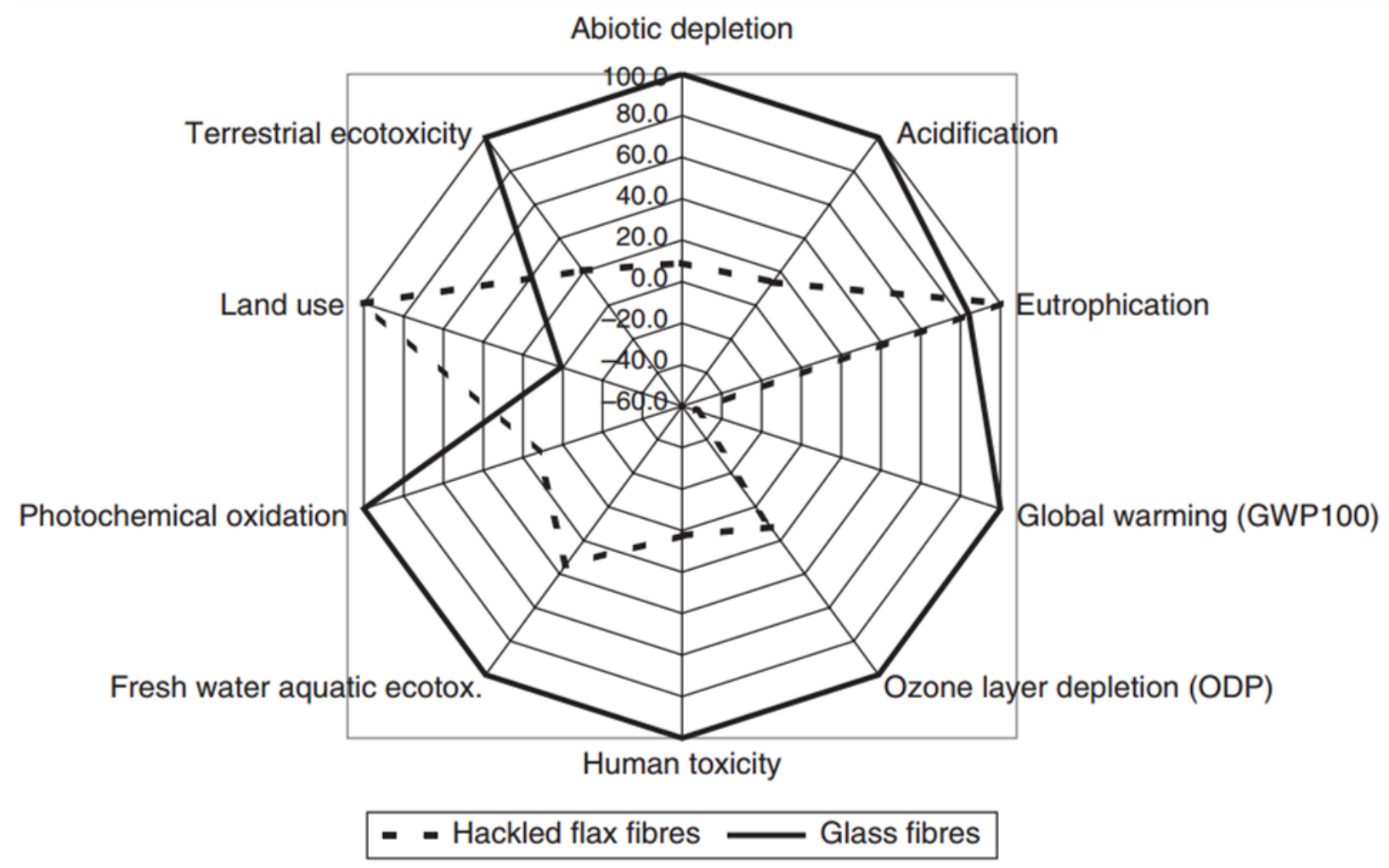

Figure 1-1: Environmental impacts from $1 \mathrm{~kg}$ fiber production, Flax vs Glass [8] 
The hybrid analysis method breaks down environmental damage into environmental indicators such as climate change, acidification, depletion, and non-renewable energy consumption. It can be seen from figure 1-1 that flax production (dash line) is favorable to the environment in all categories except for land use. Noting that land use itself is not strictly-speaking an environmental concern, and it merely indicates that for mass production of fibre, flax as a crop requires large cultivation areas in comparison to the area occupied by a glass-fibre manufacturing plant. Large cultivation areas result in a negative global warming indicator for flax. Unlike synthetic fiber production where $\mathrm{CO}_{2}$ is a biproduct, flax production consumes $\mathrm{CO}_{2}$ from the environment via photosynthesis and therefore reduces the overall quantity of greenhouse gases.

A quick survey on flax use reveals that flax composites are becoming increasingly popular for a variety of applications in major industries:

\section{Automotive}

A common automotive application of Flax is car doors panelling where flax short fibers are blended with a thermoplastic matrix such as polypropylene. The yielded blend is then compression molded into the final composite parts. Other typical applications of flax fibers include window pillars, package trays, and trunk liners [11]. Recently, CARBIO developed a carbon-flax hybrid structures for automotive applications. Mixing carbon and flax at a 50/50 ratio, results in laminates that are 15\% cheaper, $7 \%$ lower weight, and have 58\% higher vibration damping while maintaining an equal bending stiffness when compared with carbon-only laminates [12]. Moreover, Faurecia Flaxpreg won the European 2015 JEC semi-products award for a very long flax reinforced sandwich composite. Applied as structural trim parts (trunk load floor or passenger compartment structural floor), it offers excellent mechanical properties and a $35 \%$ weight reduction compared to traditional petro-based glass mats [13].

\section{Consumer Products}

Flax can be used in compression molding, injection molding, hand lay-ups, and other hybrid technologies [14]. This versatility allows flax fiber composites to become consumer products such as luggage, musical instruments, furniture, racquets, snowboards, canoes, paddle boats, and hockey sticks. Moreover, Flax has been used for more specific applications, such as improving the impact performance of the UK-based 'Urban One' bamboo composite bicycle. Using flax over carbon fiber, resulted in a stiff yet quiet and vibrationless frame [14]. 


\section{Infrastructure}

Flax has found use in commercial construction as well. Blending Flax into eco-friendly binders results in alternative panel materials to typical wood-based materials such as plywood. Flax can be blended into concrete and stucco, and flax-based insulation is gaining popularity throughout Europe. In 2010, a 100\% biodegradable vertical windmill composed of flax fabric and PolyLactic Acid (PLA) matrix won the JEC innovations award in the category of wind energy [15]. Later in 2014, University of Stuttgart manufactured flax-reinforced blades for rooftop wind turbines; aiming at improving the reliability of wind turbines, while simultaneously reducing the production cost [16].

\section{Limitations}

As seen from the above examples, Flax is making a strong entrance into modern manufacturing industries. Recent publications and ongoing research identify natural fibers as a cost-effective and environmentally beneficial substitute to synthetic fibers. However, the use of flax composites for load bearing applications is still at its infancy stage, and only some ongoing research projects are currently laying the ground for flax composites to be used in structural components. Major industries have been avoiding using NFCs for load-bearing applications due on one hand to their susceptibility to water moisture, and on the other hand to the lack of data on the mechanical performance of NFCs, particularly in compression and reliable and practical modeling tools capable of simulating NFC behaviour.

Unlike conventional composites reinforced with Glass or Carbon fibers, require further research on their mechanical properties and their long term damage and fatigue behaviour is required to advance knowledge on NFCs. Considering the fact that natural fiber-reinforced composites present an attractive alternative to high-performance glass-reinforced composites, the research embodied in this research aims to expand the body of knowledge on NFCs and their use for structural applications. This thesis focuses on characterising and modeling the compressive mechanical response of Flax/Epoxy composite laminates with emphasis on damage and plasticity evolutions. 


\subsection{Thesis Outline}

This thesis report provides a deep understanding of the mechanical behaviour of Flax/Epoxy composite laminates. It also describes in details the development and implementation of a Modified Mesoscale Damage Model capable of capturing the non-linear behaviour observed in natural fiber composites.

Chapter 1 describes the context and the scope of this research work. Chapter 2 is a critical review on the current knowledge on flax fibers and flax-reinforced composites. Key areas covered in Chapter 2 include the mechanical properties of NFCs, damage mechanisms, and modeling theories. This chapter is concluded with a clear statement of the main objectives as well as key subobjectives. Chapter 3 focuses on the methodology used to achieve the objectives highlighted in Chapter 2. This Chapter covers the manufacturing, testing, and characterization of Flax/Epoxy behaviour under compressive loads. In Chapter 4, we present the theoretical background on the modified damage model, along with the model's parameters identification and implementation. Chapter 5 is dedicated to validations, results, and deep discussion of our main findings. In the final Chapter (6), we draw some conclusions and possible future works.

Supplementary material regarding calculations and software code are found are provided in the appendices, followed by the list of references. 


\section{Chapter 2: Literature Review}

\subsection{Flax Fiber}

Originating in Mediterranean regions of Europe, Flax (Linum usitatissimum) is one of the first crops domesticated by man. Its extensive cultivation dates to ancient Egyptians, who used flax fibers to produce fine linens [17 - 19]. Commercial production started in the 1800s, as flax moved west to North America. However, the invention of the cotton gin in 1973 (a machine that easily separating cotton fiber from seed) brought a decline to flax production; and cotton became the dominant plant used for clothing-fabric production [19].

This chapter will cover the available literature in regards to flax fiber and flax composite mechanical properties, damage theories, and the overall objectives of this thesis.

\subsubsection{Structure and Mechanical Properties}

Unlike synthetic fibers, flax fibers are composed of complex hierarchical structures as seen in Figure 2-1a [20, 21]. Bast fiber bundles are $1 \mathrm{~m}$ long and 1-3 $\mathrm{mm}$ in diameter. They are composed 10 - 40 polyhedron-shaped elementary fibers held together by a pectin (glue-like) interface compose the so-called technical fiber. Elementary fibers are single plant cells consisting of a primary cell wall, secondary cell wall which surround the hollow lumen [20, 21].

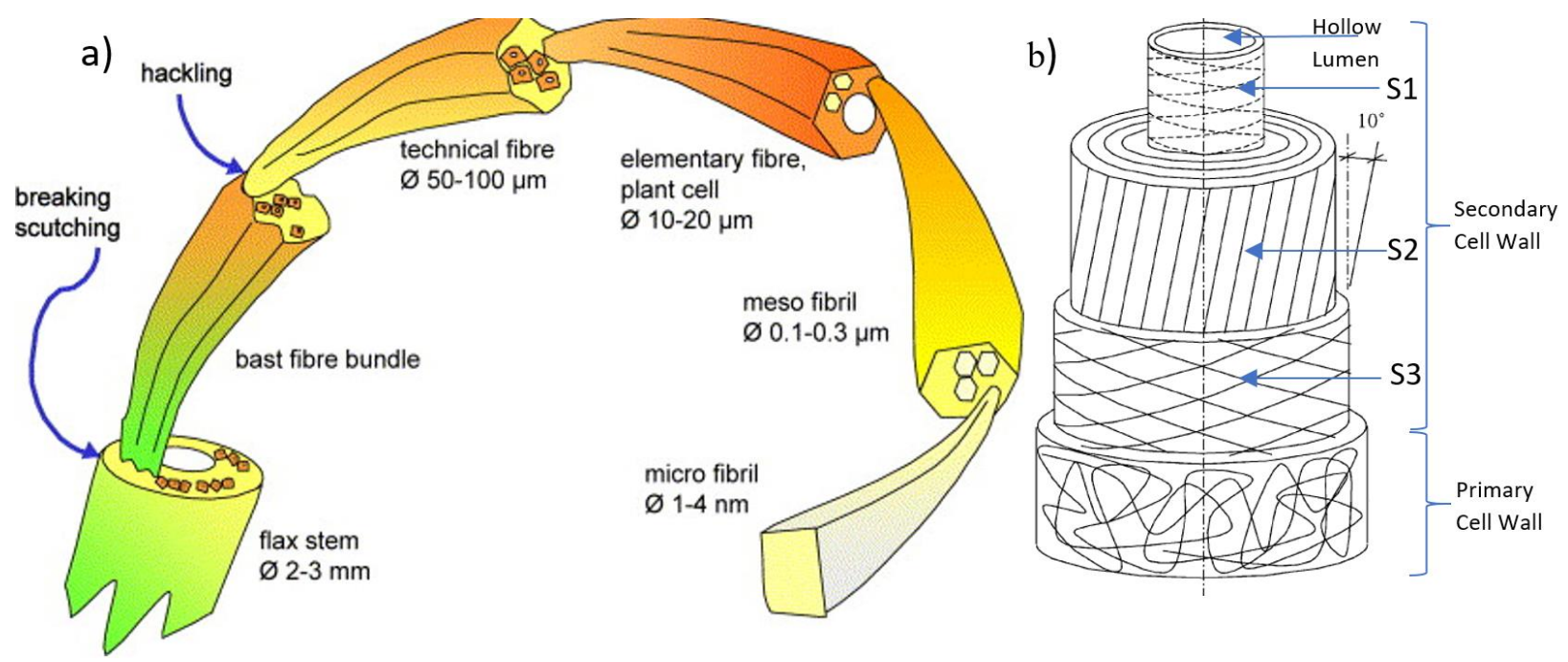

Figure 2-1: Flax stem to fiber diagram and b) elementary fiber structure [20, 21] 
Looking at the elementary fiber schematic presented in Figure $2-1 \mathrm{~b}$, the bulk of the elementary fiber are crystalline cellulose micro-fibrils (circa 70\%) bundled into meso-fibrils. Located in the S2 fiber-cell layer, the microfibrils spiral at a 10-degree orientation around the elementary fiber lumen. The microfibrils themselves are composed of non-branched glucose molecules, firmly held together by hydrogen bonds which also gives the fibers a hydrophilic nature.

Under tensile loading, flax fibers were observed to experience stiffening as well as plasticity [22 - 26]. Bayle et al [22] performed cyclic tensile testing and noticed that the microfibrils re-arrange towards the longitudinal axis with each cycle, approaching a $0^{0}$ orientation without returning to their original $10^{0}$ orientation upon unloading. This microfibril reorientation within the S2 layer is responsible for the observed change in the elastic modulus (stiffening with an increased elastic modulus by $60-80 \%$ ) as well as the permanent fiber deformation (plasticity beyond 3\% strain). This trend of increasing stiffening and plastic deformation is not unique to flax fibers; it has been observed in other natural fibers containing a similar S2 layer such as hemp [26].

When the flax fibers are loaded in compression, kink bands seen in Figure 2-2a are irreversibly produced during plant growth and/or decortication (fiber extraction procedure). They severely degrade the fibers' mechanical properties, and most often they present the fibers' weakest regions [22, 26] (Figure 2-2b [20]). Bos \& Donald [20] have used a modified loop test and observed that under compressive loads, plastic deformations occur via the development of kink bands which lead to primary cell wall failure in buckling. Similar results were found by Bos et al. [27] for hand-isolated (i.e., defect free) elementary fibers.

Depending on the study, Flax fibers have been observed to have a wide spread of mechanical properties as reported by Mahboob et al. [27]. This is mainly due to variations in fiber origin, moisture level, specimen length, heterogeneous fiber geometry, and fiber imperfections [22 - 25]. In compression, very few scarce compressive data on flax fibers have been theoretically back-calculated from composite data, while others estimated via modified loop tests as done by Bos \& Donald [20]. Even though the compressive strengths reported promises desirable mechanical characteristics, the scarcity of data reduces confidence in flax fibre compressive applications. This further solidifies the need of compressive research to be conducted on flax. 

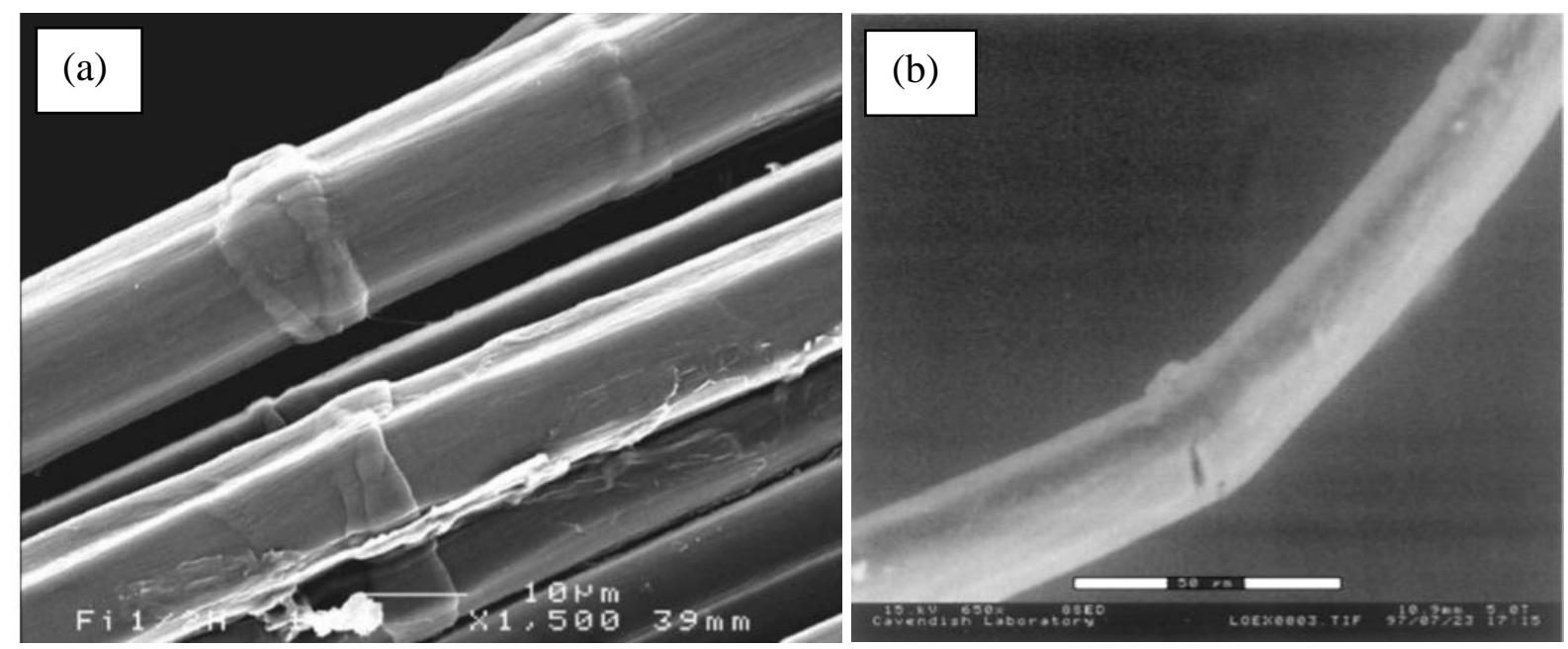

Figure 2-2: Flax fiber a) kink [22] b) transverse cracking initiating at a kink band [20]

\subsection{Flax Composites}

Natural fibers generally have a poor interfacial adhesion strength with synthetic polymers such as generic unsaturated polyester thermosets mainly due to the fiber's hydrophilic nature (i.e. susceptibility to water absorption). High performance epoxies, on the other hand, have been shown to have good adhesion strength and are a viable matrix material for NFCs [28]. Unlike conventional material such as metals and polymers, composites fail due to a combination of multiple failure mechanisms working independently or in a coupled way. Moreover, first failure does not necessarily mean final failure. A component may experience multiple micro-level failures from several independent or coupled mechanisms before becoming unfit to carry its design load. Therefore, a profound understand of both damage and its progression is required in order to be able to predict the composite's behaviour.

\subsubsection{Damage Mechanics of Flax Composites in Compression}

Local failures are referred to as damage, and their physical representations are local discontinuities. Damage evolution is defined as the development of additional local discontinuities under increasing loads, resulting in irreversible changes in the material, and degradation of material properties up to the eventual failure of load-carrying capabilities [29, 30]. Composite damage mechanisms act on two basic scales: macro and micro.

\subsubsection{Macroscale level}

These failure mechanisms are at the lamina and laminate levels. In tension, NFCs generally have non-linear and ductile properties, similar to the individual fibers. In compression, the matrix acts 
as a support to the fibbers, therefore the compressive NFC lamina strength becomes a function of both fiber and matrix properties. Here, the fiber-matrix adhesion strength prevents matrix rapture, and the matrix strength prevents fiber backing [31]. On the laminate level, residual stresses in between layers of different fiber orientations may cause delamination, where one or more layers buckle separately from the remainder of the composite.

\subsubsection{Microscale level}

Bos et al. [26] observed that matrix cracks were not observed to be a critical failure mechanism on this scale, therefore micro-scale failures are solely attributed to fibers. As previously mentioned, circumferential kink bands drastically reduce the fiber's compressive properties. Even though the primary cell wall does not actually crack in compression, failure does occur around the kink bands. Within the S2 layer, Bos et al. [26] described microfibrils coming apart much like a steel cable subjected to compressive loads. Therefore, the failure mechanism at this level is identified as intermicrofibril adhesion failure. Typical failure mechanisms of fibrous composite materials include delamination, debonding, matrix rupture, and fiber rupture pull out as shown in Fig. 2-3.
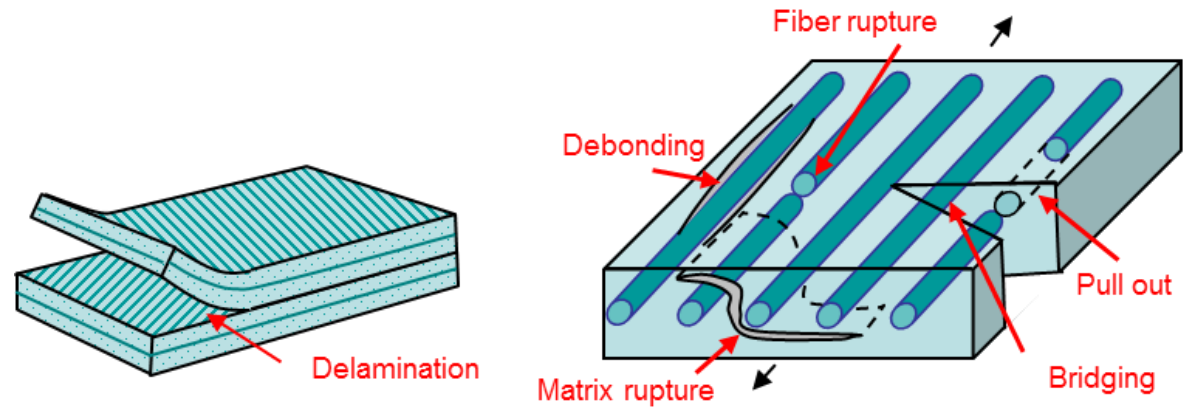

Figure 2-3: Failure modes of fibrous composite materials [32]

\subsection{Damage Theories}

Accurately modeling mechanical behaviour of NFCs requires the selection of a physicsbased theory capable of capturing damage and plasticity initiation, evolution, and failure criteria. As previously mentioned, fibrous composites tend to fail due to a combination of multiple local failures. Since failures mechanisms were identified on both micro and macroscales, a successful damage theory should encompass constitutive equations on a scale between micro and macro, also known as the Mesoscale. There are several failure prediction approaches for composite laminates, however the most popular theories are: Failure Criterion, Progressive Failure Analysis, Fracture 
Mechanics, and Continuum Damage Mechanics. The following section will discuss their strengths, weaknesses, and asses their suitability for NFC modeling.

\subsubsection{Failure criterion}

The Failure criterion approach is rather conservative, simple, straight forward, and widely used in engineering applications. The simplest form being the Maximum Stress/Strain criteria; which compares the current material stress/strain state with a pre-defined maximum limits [30]. This non-interactive approach is on the macroscopic scale, and does not consider individual stress or strain components. Well known interactive failure criteria include Tsai-Hill, Tsai-Wu, and Hashin criteria are the most popular choices in fibrous composite failure predictions. However, they are not based on the physics of damage and failure mechanisms. They are, rather based on semi-empirical relationships that correlate, to some degree, analytical predictions with experimental observations via interactive equations [29].

\subsubsection{Progressive Failure Analysis}

This method degrades a composite's material properties (typically stiffness matrix components) once a failure criterion is locally satisfied. The essence of this method is modeling sudden brittle failure by forcing appropriate stiffness matrix components to zero, once a certain failure criterion is met. Following which, stresses in the laminate are recalculated with the new stiffness matrix [30]. The main advantage of this approach is the ease of numerical implementation. However, there are two major disadvantages: (i) sudden stiffness reduction may lead to convergence issues, (ii) it does not consider effects of stress concentrations around material imperfections or sudden geometry discontinuities [28].

\subsubsection{Fracture Mechanics}

The focus of Fracture mechanics is to simulate crack propagation, and consequently, asses and quantify the conditions under which the material can fail due to dominant crack enlargement $[31,33]$. This macroscopic-scale approach is well documented and many established numerical procedures exist for a variety of composite materials. The main drawback of fracture mechanics, is that it requires an initial crack or defect to be present. Therefore, this approach cannot simulate damage initiation, only its evolution. Moreover, it focuses on one dominant crack, and does not consider the propagation and interactions of multiple small cracks [33]. 


\subsubsection{Continuum Damage Mechanics (CDM)}

Continuum Damage Mechanics (CDM) methods are developed to predict material damage initiation, progression, and failure conditions. Such methods make use of continuously evolving internal state variables and their associated thermodynamic forces to quantify damage[34]. Damage is considered irreversible, and one method of expressing its evolution is through terms of progressive deterioration of stiffness resulting in nonlinear and inelastic response as well as permanent strain formation. These CMD methods quantify the damage evolution at the microscale level, by modeling macroscale effects such as permanent strain accumulation and stiffness degradation. Due to the formulation's irreversible nature, nonlinear techniques must be employed for such approaches [27].

Considering the above discussed failure theories, it is easily noticeable that CDM-based methods provide the most comprehensive and robust mechanical response models for composite materials exhibiting nonlinear behaviour such as observed in NFCs. Moreover, it is one of the few theories capable of predicting damage initiation, evolution, and failure conditions which are crucial in designing load-bearing components.

\subsection{Standard Mesoscale Damage Theory (MDT)}

The standard Mesoscale Damage Theory proposed by Ladeveze \& Le Dantec [34] is one the most popular, robust, and versatile CDM-based theories. The Model is founded on the principle of irreversible thermodynamics, where a local material state is expressed in terms of state variable and associated thermodynamic forces.

The MDT assumes that a damaged material's mechanical response at any load state can be expressed in terms of degraded elastic moduli (damage) and accumulated permanent strains (plasticity). Since this model does not depend on time derivatives of state variables, it implies that any damage or plasticity evolution is a succession of a previously established equilibrium state.

In general, the theory considers the laminate as a composition of two elementary components: layers of composites and the interface separating them. Damage states are allowed to vary from layer to layers, however it is considered to be uniform throughout each individual lamina. The forms of damage evolution laws are considered material-dependent, since they are a reflection of the material's basic material properties and dominant damage mechanisms. The theory does not quantify individual damage mechanisms, but rather uses experimental observations to characterize 
the cumulative damage evolution. Therefore, it is phenomenological in nature and has been successfully applied to polymeric, ceramic, and metallic composites reinforced with short fibers, long fiber, or fabric.

Ladveze and Le Dantec [34] theory as it is currently formulated, however, cannot be applied to flax-reinforced composite laminates in compression. The theory is only suitable for brittle synthetic fibers such as Carbon, and explicitly mention that no fiber-direction or transversedirection damage or plasticity occur in compression. This in not true for flax composites, where compressive damage and plasticity occur and evolve with the formation of circumferential kick bands. Furthermore, the theory was developed considering a linear-evolving damage and plasticity equations in compression. The same linear evolution trends may not be necessarily true for flax composite under compressive loads. Moreover, it should be mentioned that in this model, experimental stiffness degradation is observed in compression, and is expressed using only a material specific constant which linearly correlates the compressive stiffness loss to the applied compressive stress. This indeed is an incomplete formulation to describe the damage and plasticity of flax composites, as neither damage nor plasticity are individually characterized or quantified in compression.

In order to successfully model the compressive behaviour of flax composite laminates, the standard MDT must be revisited and adopted to accurately predict the complex behaviour of flax composites. This can be done by developing new constitutive equations for damage and plasticity that encompass the experimental observations as it was shown in the work performed by Mahboob et al. [32] where new damage and plasticity equations were developed to capture the mechanical response of flax composite laminates under tensile loads.

\subsection{Thesis Objectives}

Based on the literature review presented in this chapter, it is easily seen that flax-reinforced composites are impeded from mainstream structural applications due to a lack of two key aspects: knowledge on material behaviour in compression, and robust and practical modeling tools able to capture the complex behaviour of flax composites. Therefore, the first objective of this thesis is to contribute to expand the body of knowledge on flax composites, specifically we intend to fill the gap in the literature on the compressive mechanical behaviour of flax composites. The second 
objective is to develop a physics-based predictive toll capable of capturing the non-linear mechanical behaviour exhibited by flax composites.

In order to achieve the main objectives, the following sub- objectives should be met:

\section{Manufacturing of flax test specimens of various fiber orientations.}

Great care must be taken in this step, to ensure that test specimens manufactured through hand layup and compression moulding techniques achieve comparable mechanical properties to the ones reported in published studies.

\section{Testing of Flax specimens.}

Quasi-static mechanical compressive tests must be performed on flax composites. Since raw data has a significant amount of noise associated with it, especially in compression, it must be cleaned of excessive noise prior to analysis.

\section{Analyzing test data and categorizing material behaviour trends.}

Experimental data must be analyzed according to standard procedures, in order to yield flax-specific material behaviour trends.

\section{Modification of the mesoscale model and implementation}

The mesoscale model must be modified to encompass the material behaviour trends observed in experimental tests.

\section{Model parameter identification}

Several model parameters are required to be identified. Some are possible to derive from experimental data, while others must be obtained using optimization techniques.

\section{Validation of the modified mesoscale model.}

Finally, the model must be validated on other types of composites, to ensure its accuracy and reliability.

The successful achievement of the above-mentioned goals, will provide engineers a practical tool to design components with confidence in flax-reinforced composites. This will in turn accelerate the development of NFCs for load bearing applications. 


\section{Chapter 3: Manufacturing, Quasi-Static Testing, and Observations}

\subsection{Manufacturing of Test Specimens}

There are several manufacturing techniques for composite materials such as resin transfer moulding, vacuum infusion, pultrusion, etc. The procedure used in this study to fabricate the Flax/Epoxy specimens was compression moulding. This technique is relatively simple and cost effective.

Unidirectional (UD) flax fabrics were used for composite plates manufacturing, due to the ease of cutting specimens with a variety of fiber orientations (Fig. 3-1). Flax fabrics FlaxPly® treated to an area-weight ratio of $150 \mathrm{~g} / \mathrm{cm}^{2}$ by a patented sizing and drying process (US Patent No. 8080288) were purchased from Lineo NV, Belgium. The bulk of the fabric consists of warp ( 0 deg) yarns held together by periodic weft (90 deg) yarns at a ratio of 40:3; as seen in Figure 3-1. Noting that test results have shown that weft yarns presence has negligible influence on the composite's mechanical properties. Each strand of yarn is a bundle of elementary fibers twisted to an average rate of 5 turns $/ \mathrm{cm}$.
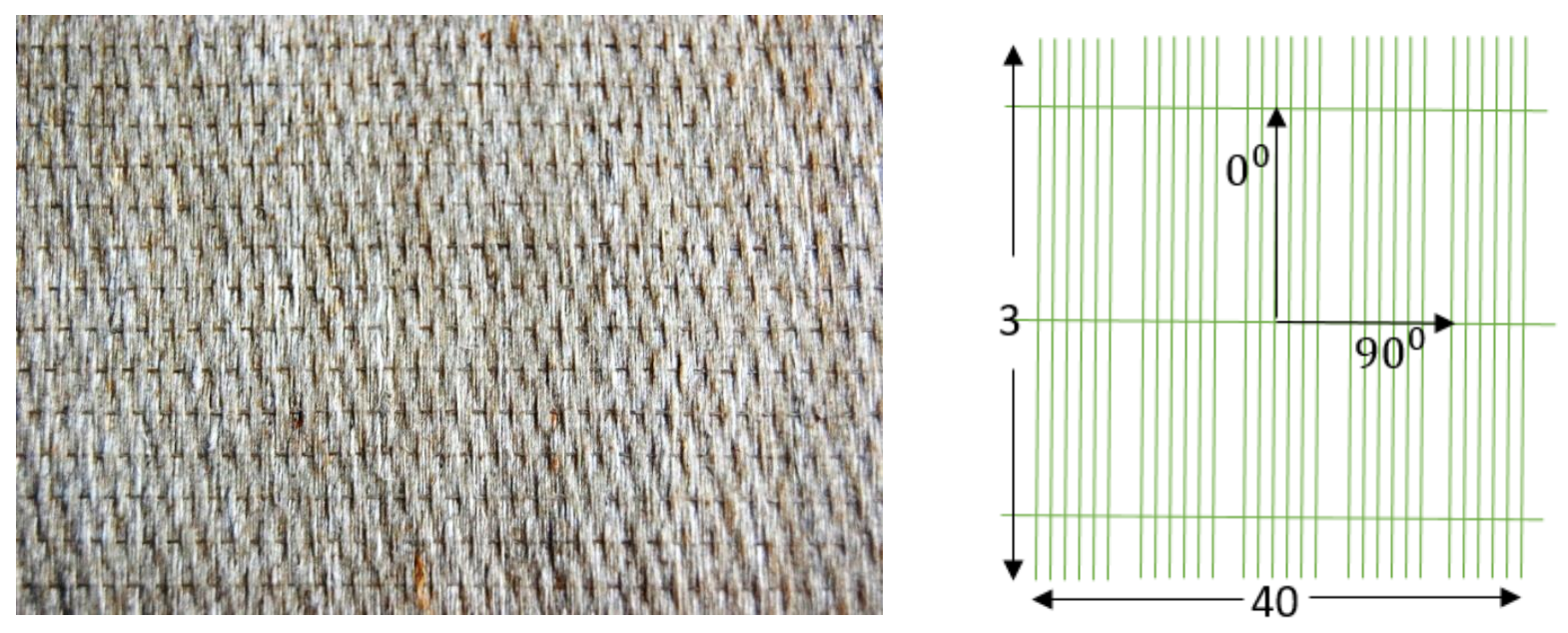

Figure 3-1: Dry flax fiber used in specimen manufacturing

As it was mentioned, natural fibers have adhesion problems with most polymer-based matrices due to the fiber's hydrophilic properties. Since this research is intended to manufacture flax composites to be used in structural applications, therefore we have chosen a matrix with superior mechanical properties. It is high performance, hot curing thermoset resin supplied by 
Huntsman Corp. It consists of Araldite® LY564 epoxy resin, and Aradur® 22962 hardener at a weight ratio of $4: 1$ respectively.

The following steps summarize the manufacturing procedure used in this study:

1. 16 layers of fabric were cut to a size of 13.5 " $\times 13.5^{\prime \prime}$, and stacked into four basic sequences $[0]_{16},[90]_{16},[ \pm 45]_{4 S},[ \pm 67.5]_{4 S}$.

2. Very good adhesion was ensured by fully soaking Flax fabrics into the Epoxy resin

3. The mould was prepared by lining the edges of 15 "x15" aluminum plates with a double layer of thick silicone. This ensured that the excess resin would escape through the silicone edges.

4. Heat-resistant peel-plies were placed on each face of the plate, to ensure clean composite extraction post curing.

5. Flax impregnated sheets were placed in the mould with the desired fiber orientation, and the matrix was poured on the fabric at intervals.

6. A fine brush was used to evenly spread the matrix and ensure uniform impregnation.

7. The mould was placed into a heated press ( Figure 3-2b) for consolidation.

8. The curing temperature cycles were set as per the manufacturer recommendations (i.e., LY 564/22962 epoxy system datasheet specifications): 15min at 120C, followed by 2 hours at 150C (Figure 3-3). 


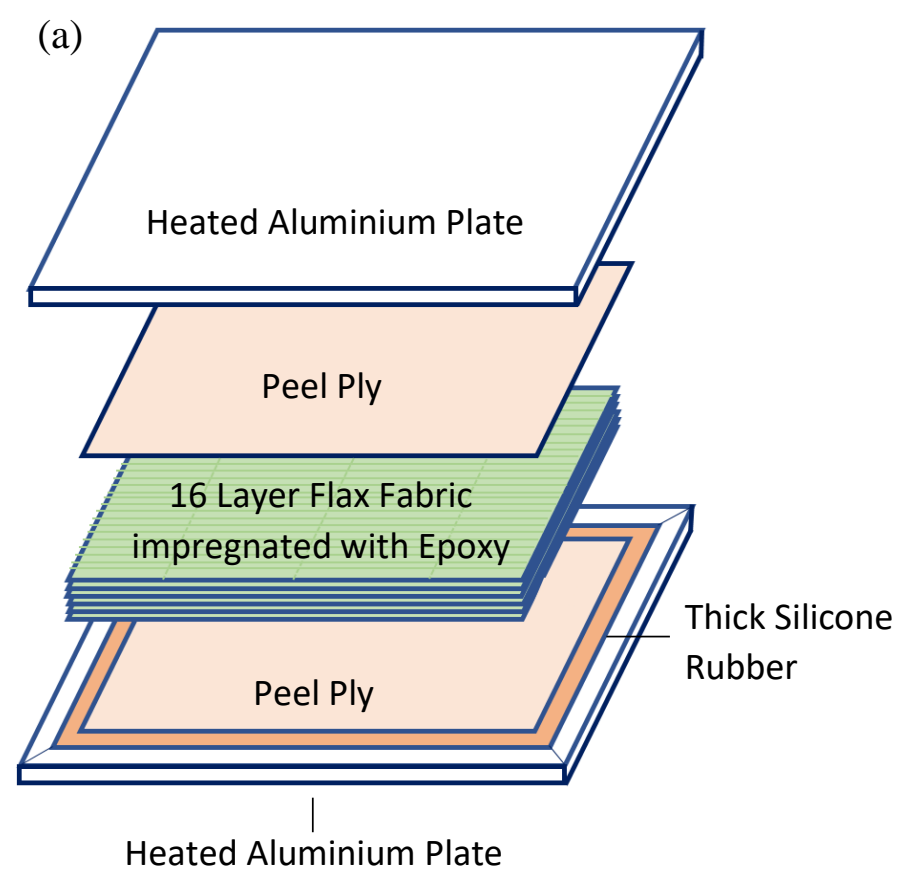

(b)

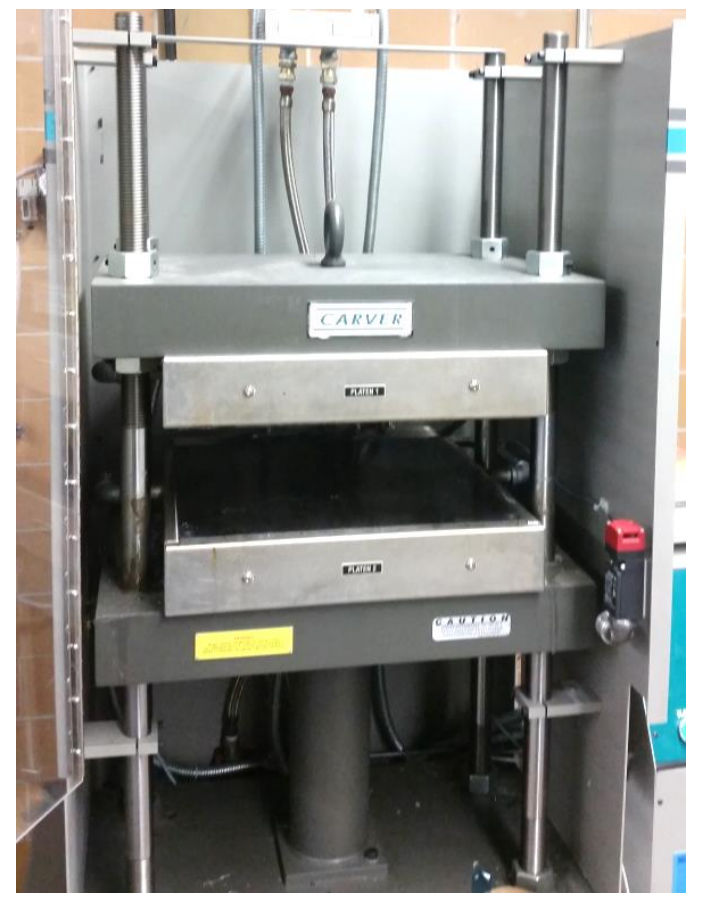

Figure 3-2: a) Hot platen press stacking sequence; b) Carver Inc. self-containing press with heating/cooling platens and programmable controller

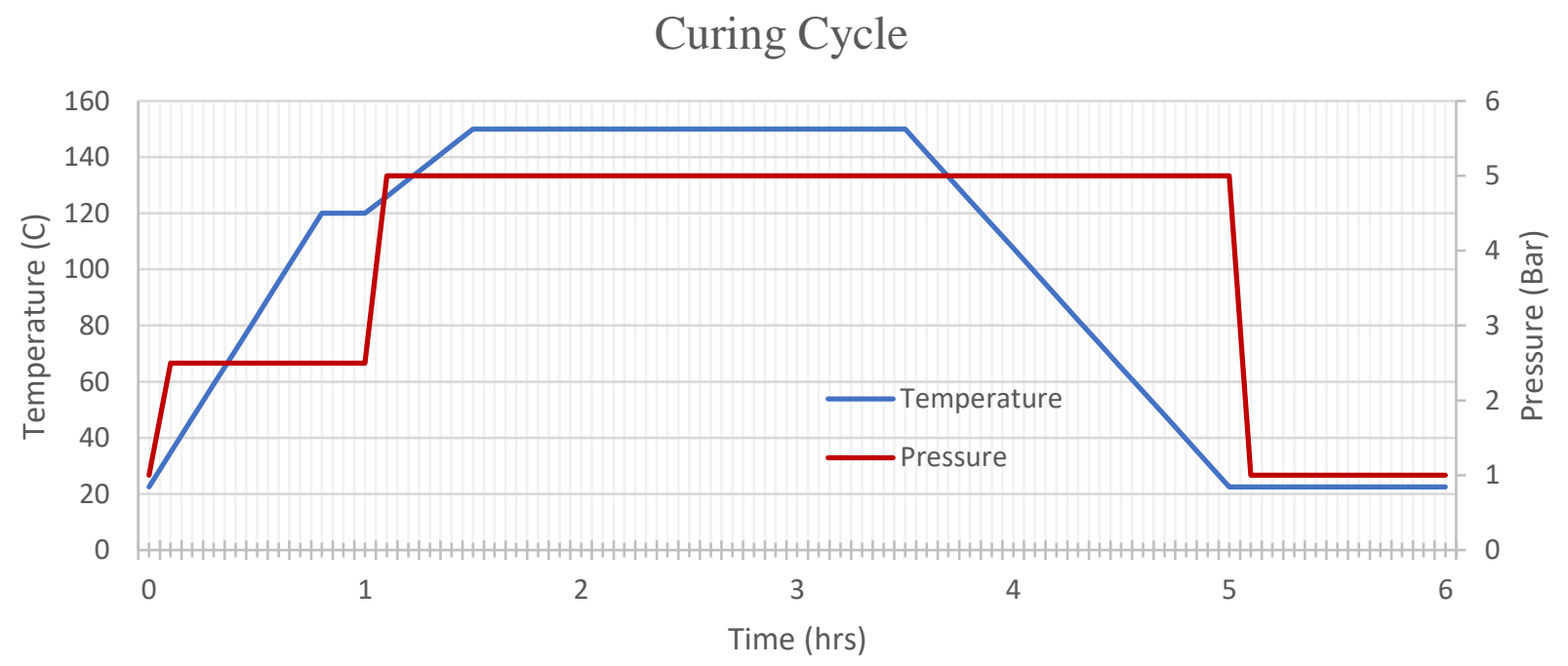

Figure 3-3: Flax composite curing cycle 


\subsection{Quasi-Static Compressive Testing}

\subsubsection{Test Specimen Preparation}

The geometry of the flax/epoxy specimens used manufactured according to ASTM D6641/D6641M guidelines [35]. Specimens with dimensions 250mm $\times 25 \mathrm{~mm} \times 4 \mathrm{~mm}$ were cut from 16-layer manufactured plates using a $0.35 \mathrm{~mm}$ diamond-edged saw (SMART CUT ${ }^{\mathrm{TM}}$, UKAM Industrial Superhead Tools, Valencia, CA, USA), and egde-ground to a flat finish. Then the specimens were tabbed using 64mm $\times 25 \mathrm{~mm} \times 3 \mathrm{~mm}$ tapered Aluminium tabs (Figure 3-4a) to avoid sllipage between the grips and the specimens during testing, and also to ensure that fractures occured in the gauge mid section of the specimens. For comparison purposes, specimens made of Epoxy only (Figure 3-4b) were manufactured by curing the matrix resin into dog-bone shapes following ASTM D695-15 standards [36].

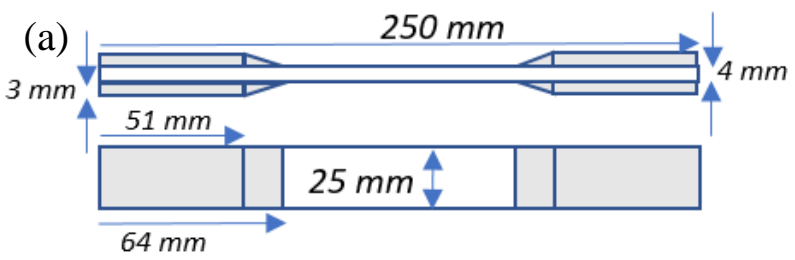

(b)

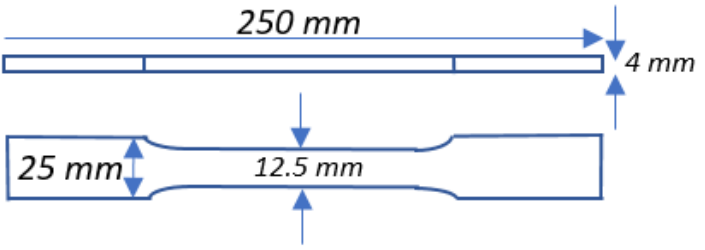

Figure 3-4: Test specimen geometry a) composite and b) matrix-only

\subsubsection{Testing Methodology}

In order to investigate damage and plasticity evolutions, repeated loading and unloading cycles were applied to the specimens with progressively increasing maximum loads up to complete failure.

Figure 3-5a illustrates the testing procedure used in this study. It is worth noting that the hysteretic response curves (stress vs strain) is approximated as linearly elastic (blue dash line). Due to the progressively increasing maximum load of each cycle, the elastic modulus degrades with each cycle, which allows for the characterize the material's damage and plasticity evolutions. The tests were conducted at room temperature using a servo-hydraulic test frame (MTS 322, Eden Prairie, MN, USA) at a displacement rate of $2 \mathrm{~mm} / \mathrm{min}$. Strain measurements were taken using a Digital Image Correlation (DIC) setup supplied by Correlated Solutions (Irmo, SC, USA). 
Specimen faces were painted with a random distribution of fine black and white speckles as seen in Figure 3-5b. The DIC camera tracks the location of each speckle in the 2D plane, while the 3D effects of specimen bulging were minimized by placing the camera at a significantly far distance. Vic $3 \mathrm{D}^{\mathrm{TM}}$ software was used to convert the speckle displacement into strain measurements. The complete testing setup used in this study is shown in Figure 3-6.

(a)

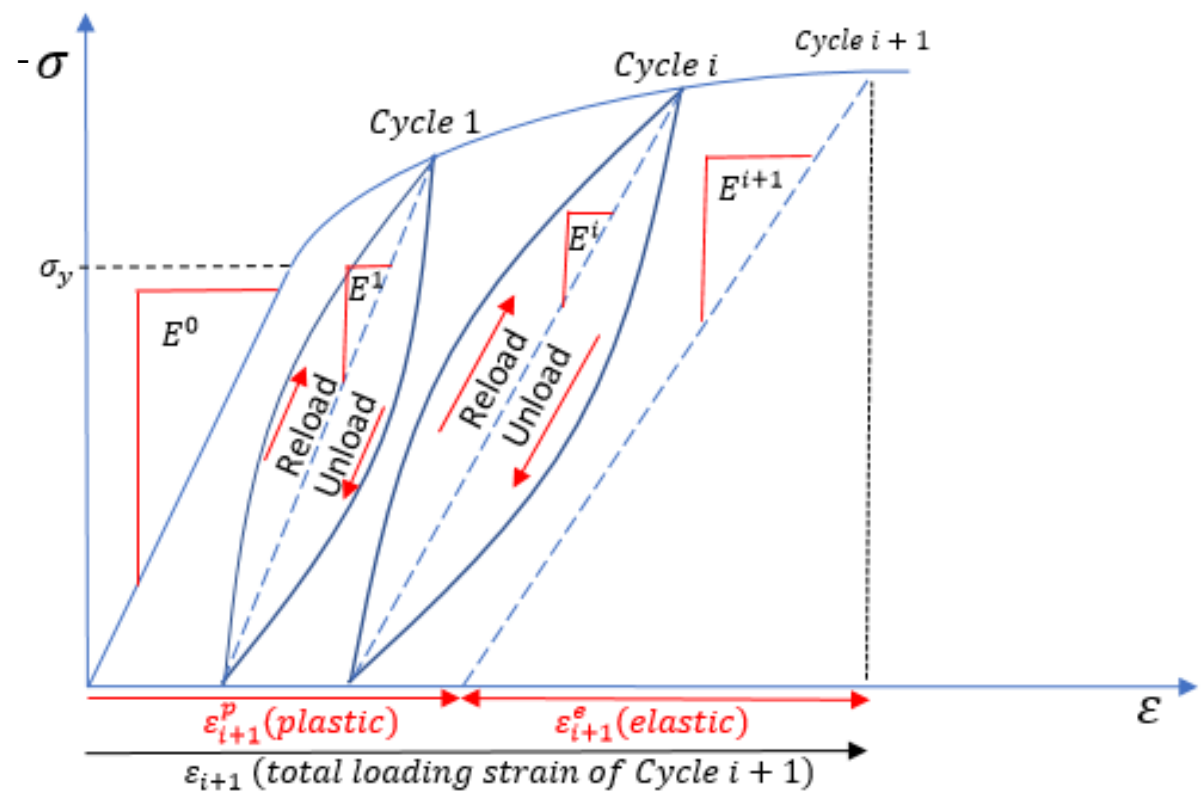

(b)

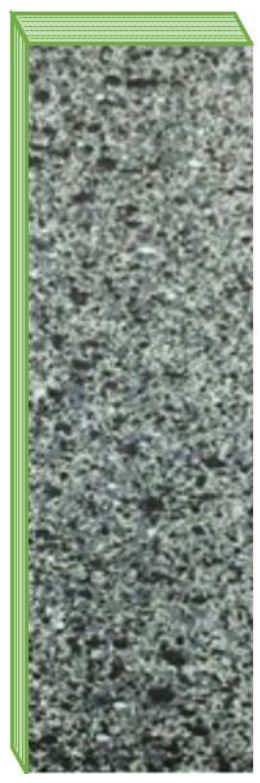

Figure 3-5: a) Testing procedure illustration, b) Test specimen with speckle pattern 

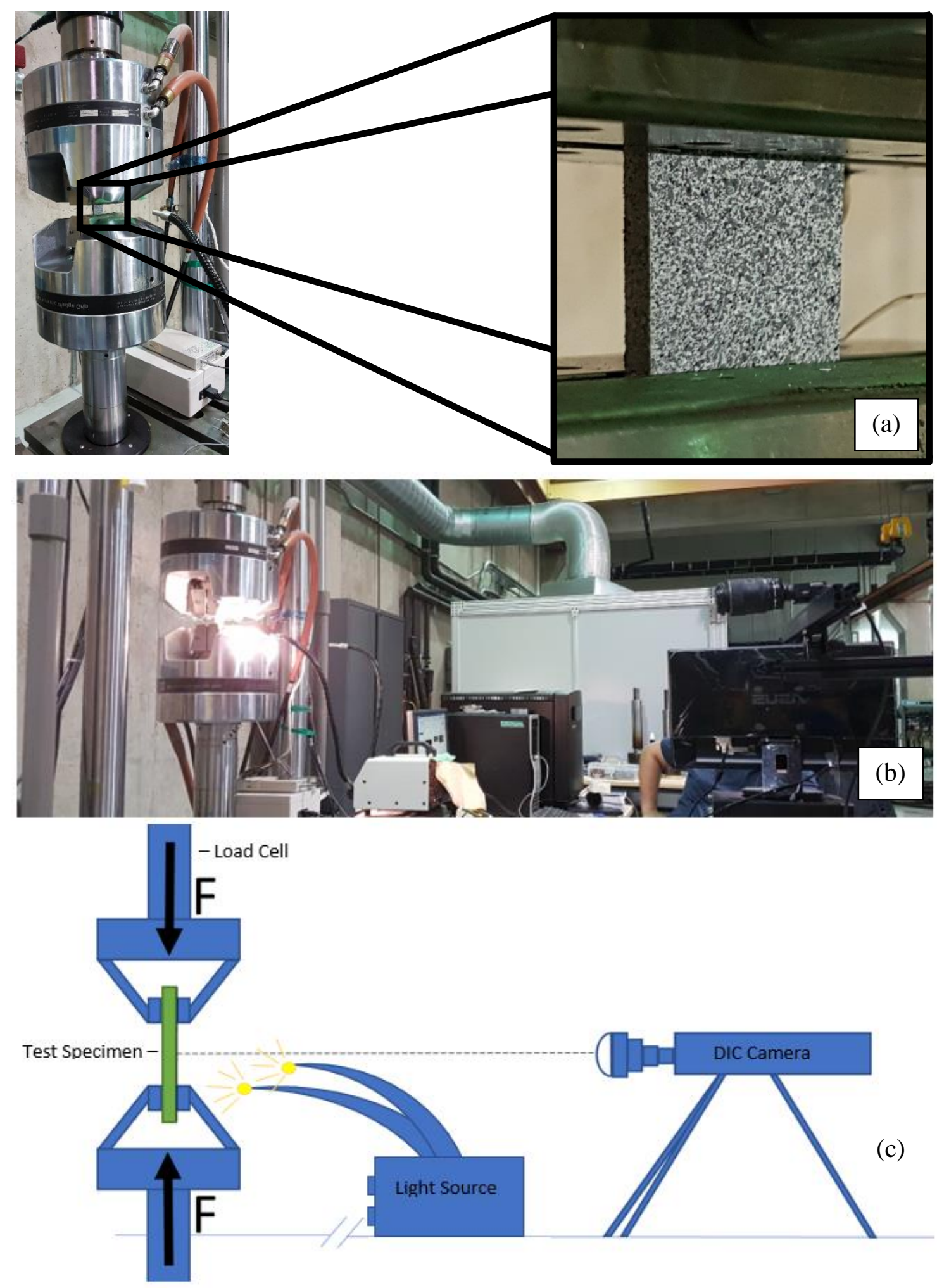

Figure 3-6: Testing procedure: a) Specimen in load cell b) Test setup c) test scheme 


\section{Chapter 4: Modified Mesoscale Damage Theory}

\subsection{Modified Mesoscale Damage Theory (MMDT)}

From the experimental data obtained from this study and the literature as well, it is clear that flax fiber composite laminates exhibit a non-linear behaviour in the fiber direction. The standard Mesoscale Damage Theory (MDT) proposed by Ladeveze \& Le Dantec [34] cannot predict this non-linear behaviour because the model assumes that both fiber and matrix fail in a brittle fashion along the fiber axis. Furthermore, the model does not consider damage or plasticity development in compression. It simply quantifies compressive stiffness loss using a materialspecific constant. Therefore, the model must be revisited in order to capture the fiber-direction, shear, and transverse compressive damage and plasticity evolutions. The following sections explain in details the standard mesoscale damage theory, and the modifications applied to it in order to account for the damage and plasticity exhibited by the flax fibers.

\subsubsection{Damage}

The MDT theory describes the damage evolution between the undamaged state of the material and macroscopic crack initiation. Consider a damaged solid within a finite volume element as shown in Figure 4-1. $S$ is defined here as the total area of the section volume element and $S_{D}$ is the total area of defects, normal to $\vec{n}$. According to Lemaitre \& Chaboche [33] damage $D$ is defined as the ratio of crack and/or void area $S_{D}$ to the total area $S$.

$$
D=\frac{S_{D}}{S}
$$

While the effective area of resistance $\tilde{S}$ in presence of damage, is defined as follows:

$$
\tilde{S}=S-S_{D}=S(1-D)
$$




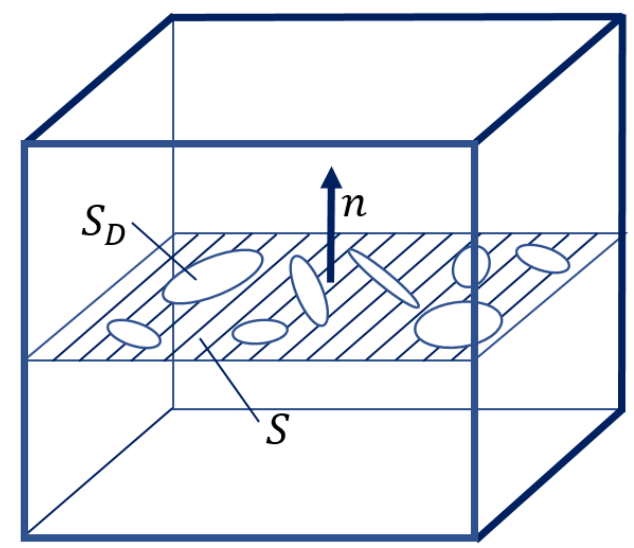

Figure 4-1: Damaged volume element

\subsubsection{Effective Stress}

Stress for unidirectional loadings is usually expressed as the ratio of force over area; i.e. $\sigma=\frac{F}{S}$. However, due to the presence of damage, the area available to resist an applied force is reduced. Therefore, the effective stress $\tilde{\sigma}$ of laminate in a state of plane stress is defined as [30]:

$$
\{\tilde{\sigma}\}=\sigma\left\{\frac{S_{D}}{S}\right\}=\left[\begin{array}{l}
\tilde{\sigma}_{11} \\
\tilde{\sigma}_{22} \\
\tilde{\sigma}_{12}
\end{array}\right]=\left\{\begin{array}{c}
\frac{\sigma_{11}}{1-D_{11}} \\
\frac{\sigma_{22}}{1-D_{22}} \\
\frac{\sigma_{12}}{1-D_{12}}
\end{array}\right\}
$$

where $D_{11}, D_{22}, D_{12}$ are the damage state parameters ranging from 0 (undamaged material) to 1 (complete damage).

At this point, it is important to note that the system of equations described by (4.3) are independent of the sign of the applied stress, meaning that there is no distinction between tension and compression. As it will be shown in the validation section, the modified theory presented here can be applied to both tensile and compressive loading cases. The original Ladeveze and LeDantec formulations [34] as well as the ones presented in the Herakovich text [30] do not account for damage evolution under compressive loads. We have modified the majority of the constitutive equations presented in [34] to account for the damage and plasticity evolutions in flax composites.

\subsubsection{Effective Strain}

The Principal of Strain Equivalence states that "any deformation behaviour, whether uniaxial or multiaxial, of a damaged material is expressed by the constitutive laws of the virgin 
material in which the usual stress is replaced by the "effective stress" [33]. Thus, the elastic constitutive equations for a damaged orthotropic material in plane stress are presented by Herakovich [30] and modified to be non-sign dependant and capable of capturing damage in both tensile and compressive cases:

$$
\begin{gathered}
\varepsilon_{11}^{E}=\frac{\left\langle\sigma_{11}\right\rangle_{+}}{E_{11}^{0}\left(1-D_{11}\right)}+\frac{\left\langle\sigma_{11}\right\rangle_{-}}{E_{11}^{0}}-\frac{\nu_{12}^{0}}{E_{11}^{0}} \sigma_{22} \rightarrow \varepsilon_{11}^{E}=\frac{\sigma_{11}}{E_{11}^{0}\left(1-D_{11}\right)}-\frac{\nu_{12}^{0}}{E_{11}^{0}} \sigma_{22} \\
\varepsilon_{22}^{E}=\frac{\left\langle\sigma_{22}\right\rangle_{+}}{E_{22}^{0}\left(1-D_{22}\right)}+\frac{\left\langle\sigma_{22}\right\rangle_{-}}{E_{22}^{0}}-\frac{\nu_{12}^{0}}{E_{11}^{0}} \sigma_{11} \rightarrow \varepsilon_{22}^{E}=\frac{\sigma_{22}}{E_{22}^{0}\left(1-D_{22}\right)}-\frac{\nu_{12}^{0}}{E_{11}^{0}} \sigma_{11} \\
\varepsilon_{12}^{E}=\frac{\sigma_{12}}{2 G_{12}^{0}\left(1-D_{12}\right)}
\end{gathered}
$$

where

$$
\begin{aligned}
& \langle a\rangle_{+}=a \text { if } a \geq 0 ; \text { otherwise }\langle a\rangle_{+}=0 \\
& \langle a\rangle_{-}=a \text { if } a \leq 0 ; \text { otherwise }\langle a\rangle_{-}=0
\end{aligned}
$$

\subsubsection{Quantifying Damage}

From our recently published experimental results on flax composite laminates [27], it was shown that the flax specimens have an orthotropic behaviour under both tensile and compressive loading. It was also shown that the ratio of Poisson ratio over the elastic modulus remained constant during damage evolution:

$$
\frac{v_{12}}{E_{11}}=\frac{v_{12}^{0}}{E_{11}^{0}}=\frac{v_{21}^{0}}{E_{22}^{0}}
$$

The damaged elastic moduli can be expressed in terms of damage parameters $\left(D_{i}\right)$ and the undamaged moduli $\left(E_{i}^{0}\right)$ as follows [33]:

$$
\begin{aligned}
& E_{11}=E_{11}^{0}\left(1-D_{11}\right) \\
& E_{22}=E_{22}^{0}\left(1-D_{22}\right) \\
& G_{12}=G_{12}^{0}\left(1-D_{12}\right)
\end{aligned}
$$


From (4.6), we can express the damage in the principal directions of the lamina as follows:

$$
\begin{aligned}
& D_{11}=1-\frac{E_{11}}{E_{11}^{0}} \\
& D_{22}=1-\frac{E_{22}}{E_{22}^{0}} \\
& D_{12}=1-\frac{G_{12}}{G_{12}^{0}}
\end{aligned}
$$

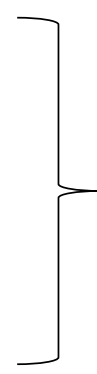

To quantify the damage and track its evolution, compressive load-unload tests as shown in Figure 3-5a, will be performed at each load cycle.

\subsubsection{Thermodynamic Forces}

The foundation of the Mesoscale Damage Theory lies in expressing a damaged layer's mean value of strain energy density $E_{D}$ in terms of effective stresses. Based on the Gibbs Free Energy equation, Herakovich [30] expressed the strain energy density function as follows:

$$
2 E_{D}=\frac{\left\langle\sigma_{11}\right\rangle_{+}^{2}}{E_{1}^{0}\left(1-D_{11}\right)}+\frac{\left\langle\sigma_{11}\right\rangle_{-}^{2}}{E_{1}^{0}}-2 \frac{\nu_{12}^{0}}{E_{1}^{0}} \sigma_{11} \sigma_{22}+\frac{\left\langle\sigma_{22}\right\rangle_{+}^{2}}{E_{2}^{0}\left(1-D_{22}\right)}+\frac{\left\langle\sigma_{22}\right\rangle^{2}}{E_{2}^{0}}+\frac{\sigma_{12}^{2}}{G_{12}^{0}\left(1-D_{12}\right)}
$$

Mahboob et al. [32] have adopted equation (4.8) to a volumetric element (i.e., 3D configuration) resulting in the following equation:

$$
\begin{aligned}
2 E_{D} & =\frac{\left\langle\sigma_{11}\right\rangle_{+}^{2}}{E_{11}^{0}\left(1-D_{11}\right)}+\frac{\left\langle\sigma_{11}\right\rangle_{-}^{2}}{E_{11}^{0}}-2 \frac{v_{12}}{E_{11}} \sigma_{11} \sigma_{22}-2 \frac{v_{13}}{E_{11}} \sigma_{11} \sigma_{33}+\frac{\left\langle\sigma_{22}\right\rangle_{+}^{2}}{E_{22}^{0}\left(1-D_{22}\right)}+\frac{\left\langle\sigma_{22}\right\rangle_{-}^{2}}{E_{22}^{0}}- \\
& -2 \frac{v_{32}}{E_{33}} \sigma_{22} \sigma_{33}+\frac{\sigma_{33}^{2}}{E_{33}}+\frac{\sigma_{12}^{2}}{G_{12}^{0}\left(1-D_{12}\right)}+\frac{\sigma_{13}^{2}}{G_{13}}+\frac{\sigma_{23}^{2}}{G_{23}}
\end{aligned}
$$

A detailed derivation of equation (4.9) can be found in [29].

In order to have an expression encompassing both tensile and compressive loads, the sign dependency was removed from equation (4.9), and the final mean strain energy density function used for flax composite compressive modeling is expressed as follows:

$$
\begin{aligned}
2 E_{D}= & \frac{\sigma_{11}^{2}}{E_{11}^{0}\left(1-D_{11}\right)}-2 \frac{v_{12}}{E_{11}} \sigma_{11} \sigma_{22}-2 \frac{v_{13}}{E_{11}} \sigma_{11} \sigma_{33}+\frac{\sigma_{22}^{2}}{E_{22}^{0}\left(1-D_{22}\right)}-2 \frac{v_{32}}{E_{33}} \sigma_{22} \sigma_{33}+ \\
& +\frac{\sigma_{33}^{2}}{E_{33}}+\frac{\sigma_{12}^{2}}{G_{12}^{0}\left(1-D_{12}\right)}+\frac{\sigma_{13}^{2}}{G_{13}}+\frac{\sigma_{23}^{2}}{G_{23}}+\frac{\sigma_{33}^{2}}{E_{33}}+\frac{\sigma_{12}^{2}}{G_{12}^{0}\left(1-D_{12}\right)}+\frac{\sigma_{13}^{2}}{G_{13}}+\frac{\sigma_{23}^{2}}{G_{23}} \mathrm{~s}
\end{aligned}
$$

The thermodynamic forces $Y_{i}$ (a.k.a. damage energy release rates), associated with the internal damage variables $D_{i}$, are defined by Lemaitre and Chaboche [33] in terms of mean value 
of the strain energy density $E_{D}$, at a current state of stress and damage. These forces govern the damage development in a way that a certain damage threshold must be exceeded for an additional damage to occur. These thermodynamic forces are expressed in [29, 30, 34] as partial time derivatives of the mean energy strain density function, with respect to the corresponding damage. Again, the sign dependence was removed as seen in previous equations:

$$
\begin{gathered}
Y_{11}=\left.\frac{\delta E_{D}}{\delta D_{11}}\right|_{\widetilde{\sigma}, D_{22}, D_{12}=\text { const }}=\frac{\left\langle\sigma_{11}\right\rangle_{+}^{2}}{2 E_{11}^{0}\left(1-D_{11}\right)^{2}} \rightarrow \frac{\sigma_{11}^{2}}{2 E_{11}^{0}\left(1-D_{11}\right)^{2}} \\
Y_{22}=\left.\frac{\delta E_{D}}{\delta D_{22}}\right|_{\widetilde{\sigma}, D_{11}, D_{12}=\text { const }}=\frac{\left\langle\sigma_{22}\right\rangle_{+}^{2}}{2 E_{22}^{0}\left(1-D_{22}\right)^{2}} \rightarrow \frac{\sigma_{22}^{2}}{2 E_{22}^{0}\left(1-D_{22}\right)^{2}} \\
Y_{12}=\left.\frac{\delta E_{D}}{\delta D_{12}}\right|_{\widetilde{\sigma}, D_{11}, D_{22}=\text { const }}=\frac{\sigma_{12}^{2}}{2 G_{12}^{0}\left(1-D_{12}\right)^{2}}
\end{gathered}
$$

\subsubsection{Damage Evolution}

The form of Damage Evolution laws proposed by Ladeveze \& Le Dantec [34] are material dependent, and have been shown effective for orthotropic material, such as fibrous composites. As previously stated, standard MDT formulations assume that the material behaves in an elastic and brittle manner along the fiber direction, meaning that there is no damage evolution in the fiber direction, and that the material is damage free up to complete failure. Since the degradation of the elastic molus has been observed in flax-fiber composites, one of the major modifications done on Ladeveze \& LeDantec model was to completely decouple the damage in fiber-direction and plasticity evolution from the shear and transverse directions. Thus, similar to the formulation seen in [29], the fiber direction and the shear-transverse direction damage development equations are completely decoupled as follows:

$Y_{f}=\sqrt{Y_{11}}(\sqrt{M P a})$ Fiber damage and fracture

$Y_{t s}=\sqrt{Y_{12}+b_{t s} Y_{22}}(\sqrt{M P a})$ Matrix cracking \& fiber - matrix debonding 
where subscripts $f$ and $t s$ represent fiber and transverse $\&$ shear directions respectively, while the coupling parameter $b_{t s}$ expresses the shear and transverse coupling effects on the fiber-matrix debonding.

From experimental observations (Section 5.2.2.1), it can be seen that the damage in $0^{0}$ direction (fibers oriented in the longitudinal direction) is a linear function and can be expressed in terms of damage function as follows:

$$
\Phi_{D_{11}}=\frac{Y_{f}-Y_{f}^{0}}{Y_{f}^{C}}-D_{11} \quad \leq 0, \quad \varepsilon_{11}<\varepsilon_{11}^{\max }
$$

For the transverse specimens ( $90^{\circ}$ fiber-oriented samples), the damage evolution equation that best fitted the experimental values has an exponential trend. However, a linear damage evolution has proven to also provide a sufficiently good fit. Therefore, and for the sake of practicality, a linear fit was used for transverse damage evolution, which also has advantages regarding computational simplicity.

$$
\Phi_{D_{22}}=\frac{Y_{t s}-Y_{t}^{0}}{Y_{t}^{C}}-D_{22} \quad \leq 0, \quad Y_{22}<Y_{22}^{\max }
$$

And, for shear damage evolution was observed to be linear and formulated similar to the transverse case:

$$
\Phi_{D_{12}}=\frac{Y_{t s}-Y_{s}^{0}}{Y_{s}^{C}}-D_{12} \quad \leq 0, \quad Y_{12}<Y_{12}^{\max }
$$

In the above equations (4.13-4.15), $\Phi_{D_{i j}}$ is the damage function of the corresponding damage $D_{i j}$; while, $Y_{f}^{0}, Y_{f}^{C}, Y_{t}^{0}, Y_{t}^{C}, Y_{s}^{0}, Y_{s}^{C}, Y_{22}^{\max }$, and $Y_{12}^{\max }$ are material-specific parameters to be determined experimentally.

\subsubsection{Inelastic Strain}

Most engineering materials exhibit a linearly-elastic response up to a certain yield limit $\sigma^{y}$. Once loaded beyond this limit, there will be inelastic or permanent strains $\varepsilon_{i j}^{p}$ present upon complete unloading. Though some hysteresis is present in the material response, the unloading/reloading behaviour may be expressed as linearly elastic as seen in Figure 4-2, where $E^{0}$ is the undamaged modulus. 


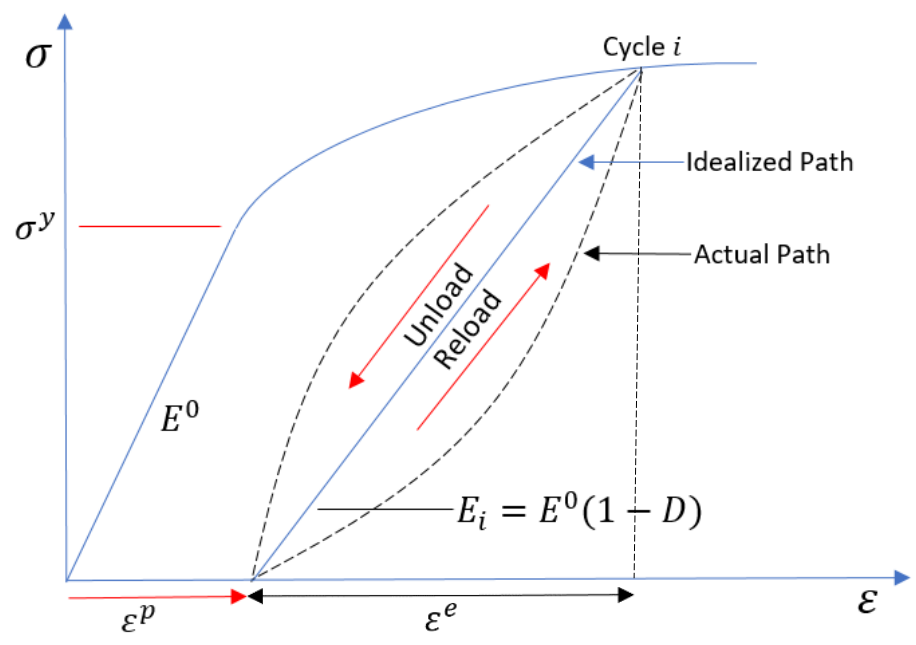

Figure 4-2: Damaged material inelastic response

The classical formulation for plasticity was also adopted in the modified model proposed in this study. The total strains are decomposed into elastic $\varepsilon^{e}$ and plastic $\varepsilon^{p}$ strains. The elastic domain function $f\left(\tilde{\sigma}_{i j}, \tilde{p}\right)$ depends on the effective stress $\tilde{\sigma}_{i j}$ and the accumulated strain $(\tilde{p})$.

The accumulated plastic strain $\tilde{p}$ is composed of two components, one for shear, and the second for transverse plastic strains $\left(\tilde{p}_{t s}=\widetilde{\varepsilon_{t}^{P}}+\widetilde{\varepsilon_{s}^{P}}\right)$.

The strain rates components $\left(\dot{\varepsilon}_{i j}^{e}+\dot{\varepsilon}_{i j}^{p}\right)$ are normal to the elastic domain function are as illustrated in Figure 4-3a, for a loading in the $\left(\tilde{\sigma}_{12}-\tilde{\sigma}_{22}\right)$ stress space. Noting that the effective inelastic strain rates are defined for all 3 the orthotropic directions as follows [29]:

$$
\widetilde{\dot{\varepsilon}_{l j}^{p}}=\dot{\varepsilon}_{i j}^{p}\left(1-D_{i j}\right), \quad \text { for } \quad i, j \in\{1,2\}
$$

As strain hardening occurs, it is assumed that the initial elastic domain labeled as $f(\tilde{p}=0)$ evolves according to an isotopic power law into the plastic domain $f(\tilde{p}>0)$ as illustrated in Figure 4-3b. The elastic domain function can be written as:

$$
\begin{gathered}
f=\tilde{\sigma}_{11}+R(\tilde{p})-R_{0}-\text { fiber direction } \\
f=\sqrt{\tilde{\sigma}_{12}^{2}+A_{t s} \tilde{\sigma}_{22}^{2}}+R(\tilde{p})-R_{0}-\text { shear-transverse }
\end{gathered}
$$

where, $R(\tilde{p})$ is the power law hardening function, $R_{0}$ represents the initial threshold value for $R(\tilde{p}=0)$, and $A_{t s}$ is the material coupling constant. 
Again, from the experimental observations in (Section 5.2.2), it can be seen that plasticity for both longitudinal and shear-transverse directions evolves according to a power law. Thus, we can express the hardening function by setting equation (4.17) as follows:

$$
\begin{gathered}
R(\tilde{p})+R_{0}=\frac{\sigma_{11}}{1-D_{11}}=\left(\beta \tilde{p}^{\alpha}-R_{0}\right)_{f} \text {-longitudinal or fiber direction } \\
R(\tilde{p})+R_{0}=\sqrt{\frac{\sigma_{12}^{2}}{\left(1-D_{12}\right)^{2}}+\frac{A_{t s} \sigma_{22}^{2}}{\left(1-D_{22}\right)^{2}}}=\left(\beta \tilde{p}^{\alpha}-R_{0}\right)_{t s}-\text { shear transverse }
\end{gathered}
$$

where $A_{t s}$ is a coupling parameter and $\alpha$ and $\beta$ are material parameters.

a)

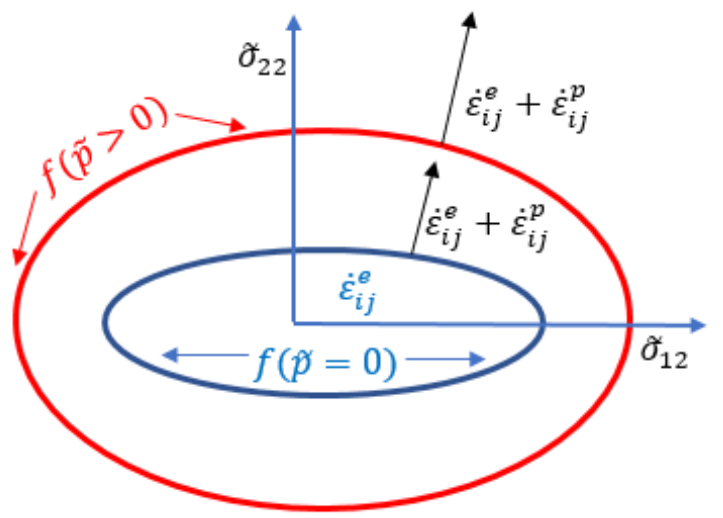

b)

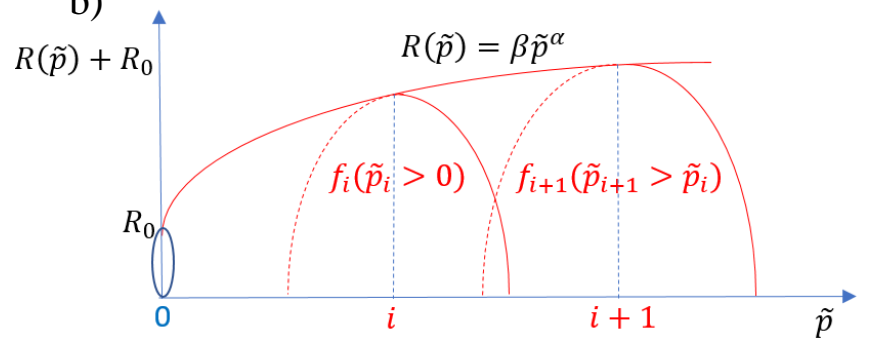

Figure 4-3: a) Shear-transverse elastic domain function, b) power-law shaped sheartransverse elastic domain evolution

Most reinforcing fibers exhibit linear-elastic response. Thus, the effects of plasticity are generally associated with the matrix. This however, is not the case for flax composites. The inelastic Flax/Epoxy response is similarly formulated as the damage, using a set of elastic domain functions $\left\{\Phi^{p}\right\}[29]$ :

$$
\begin{aligned}
& \Phi_{f}^{p}=\tilde{\sigma}_{f}^{e q}-h_{f}\left(\tilde{p}_{f}\right)-\sigma_{f}^{0} \quad \leq 0, \quad \tilde{p}_{f} \geq 0 \\
& \Phi_{t s}^{p}=\tilde{\sigma}_{t s}^{e q}-h_{t s}\left(\tilde{p}_{t s}\right)-\sigma_{t s}^{0} \quad \leq 0, \quad \tilde{p}_{t s} \geq 0
\end{aligned}
$$

where,

$\Phi_{f}^{p}$ is inelastic behaviour function in the fiber direction, $\Phi_{t s}^{p}$ is the inelastic behaviour function in the transverse-shear direction, $\sigma_{f}^{0}, \sigma_{t s}^{0}$ are plasticity initiation thresholds for fiber and sheartransverse directions respectively, and $h_{f}\left(\tilde{p}_{f}\right) \& h_{t s}\left(\tilde{p}_{t s}\right)$ are the hardening functions which 
dependent on the fiber-direction and shear-transverse accumulated effective inelastic strains $\tilde{p}_{f}$ and $\tilde{p}_{t s}$.

In the case of Flax composites, both hardening functions fit the shape of power curves, therefore the hardening function can be expressed as follows:

$$
\begin{gathered}
h_{f}\left(\tilde{p}_{f}\right)=\beta_{f}\left(\tilde{p}_{f}\right)^{\alpha_{f}} \\
h_{t s}\left(\tilde{p}_{t s}\right)=\beta_{t s}\left(\tilde{p}_{t s}\right)^{\alpha_{t s}}
\end{gathered}
$$

Here the material parameters $\alpha$ and $\beta$ have different values for fiber and shear-transverse directions.

Lastly, are equivalent stress-based scalars $\tilde{\sigma}_{f}^{e q}$ and $\tilde{\sigma}_{t s}^{e q}$ that influence plasticity in their corresponding directions are expressed as follows:

$$
\begin{gathered}
\tilde{\sigma}_{f}^{e q}=\frac{\sigma_{11}}{1-D_{11}} \\
\tilde{\sigma}_{t s}^{e q}=\sqrt{\frac{\sigma_{12}^{2}}{\left(1-D_{12}\right)^{2}}+\frac{A_{t s} \sigma_{22}^{2}}{\left(1-D_{22}\right)^{2}}}
\end{gathered}
$$

\subsection{Parameter Identification}

In total, this model uses twenty-one material-specific parameters. Some represent the material basic properties including elastic moduli, poison's ratios, maximum strains, and onset of damage and plasticity threshold, usually determined directly from experimental data. These basic parameters are then fed to the mathematical model. The other parameters related to damage and plasticity evolution equations were estimated by the modified mesoscale damage model. Out of the twenty-one parameters, only two of them the shear-transverse coupling parameters $b$ and $A_{t s}$ could not be derived. They were obtained using trial-error methods. In the sections below, we will describe the procedure used to determine the MMDM Flax/Epoxy compressive parameters.

\subsubsection{Microscopic Image Analysis}

Conventional methods used for material constituent fractions evaluations such as specified by ASTM D3171 [37] involve matrix digestion in acids or incineration. These techniques are not suitable for natural fibers, since the fibers will degrade along with the matrix. Therefore, our 
research team directed by Dr. Bougherara has implemented an alternative technique using Microscopic Image Analysis in order to determine fiber, matrix, and void fractions in a composite. This technique was also used by Phillips et al. [38] and El Sawi et al [39] to determine crack/void density in flax-fiber composites.

The following steps summarize the microscopic image analysis procedure:

- Samples (up to 6) of size $25 \times 25 \mathrm{~mm}$ were cut from manufactured plates at random locations.

- ASTM E2015 [40] standard procedures were used in sample preparation for microstructural analysis.

- Grayscale microscopic images were taken via SEM (using JEOL JSM-6380, Tokyo, Japan).

- Open source software ImageJ was used to determine the area fractions by binarizing images to distinguish features of interest, and using a threshold to isolate cracks as seen in Figure 4-5, repainted with permission from [27]. It was assumed that the area fractions are representative of the volume fractions.

The constituent fractions were found to be $50.97 \%( \pm 3.92 \%)$, and $3.35 \%( \pm 2.62 \%)$ for Flax fiber and voids/cracks respectfully.
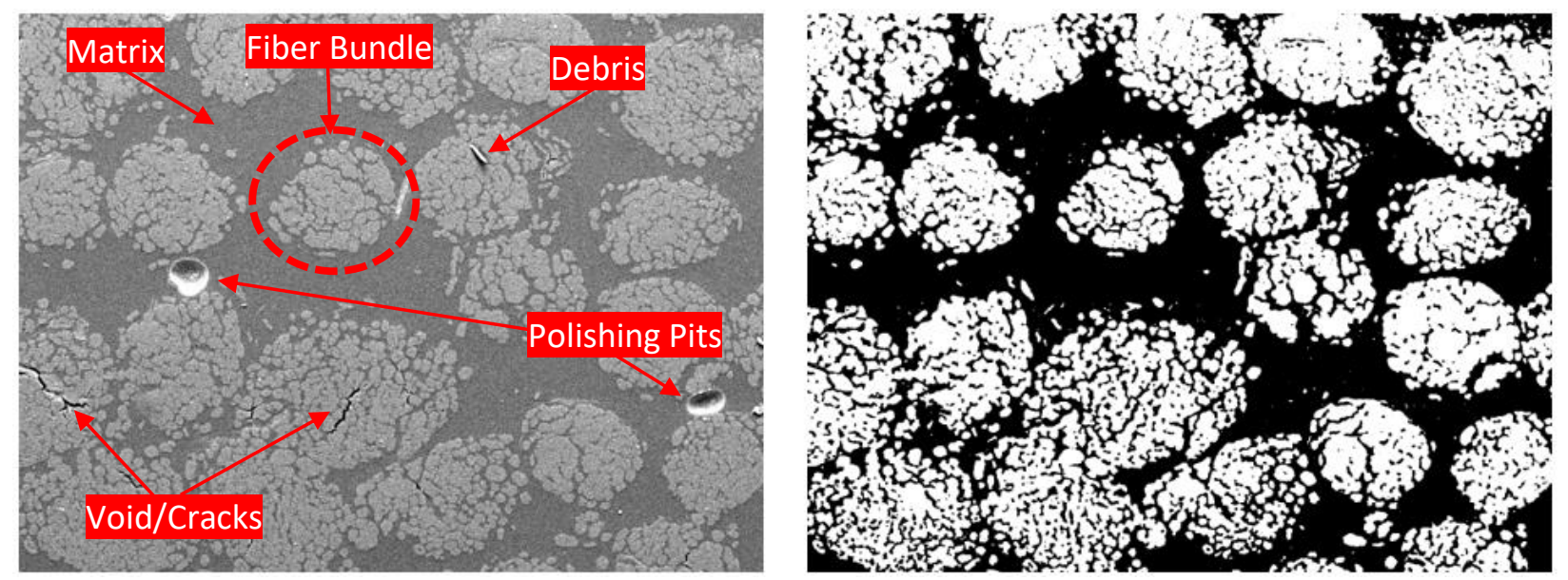

Figure 4-4: Grayscale SEM and binarized SEM 

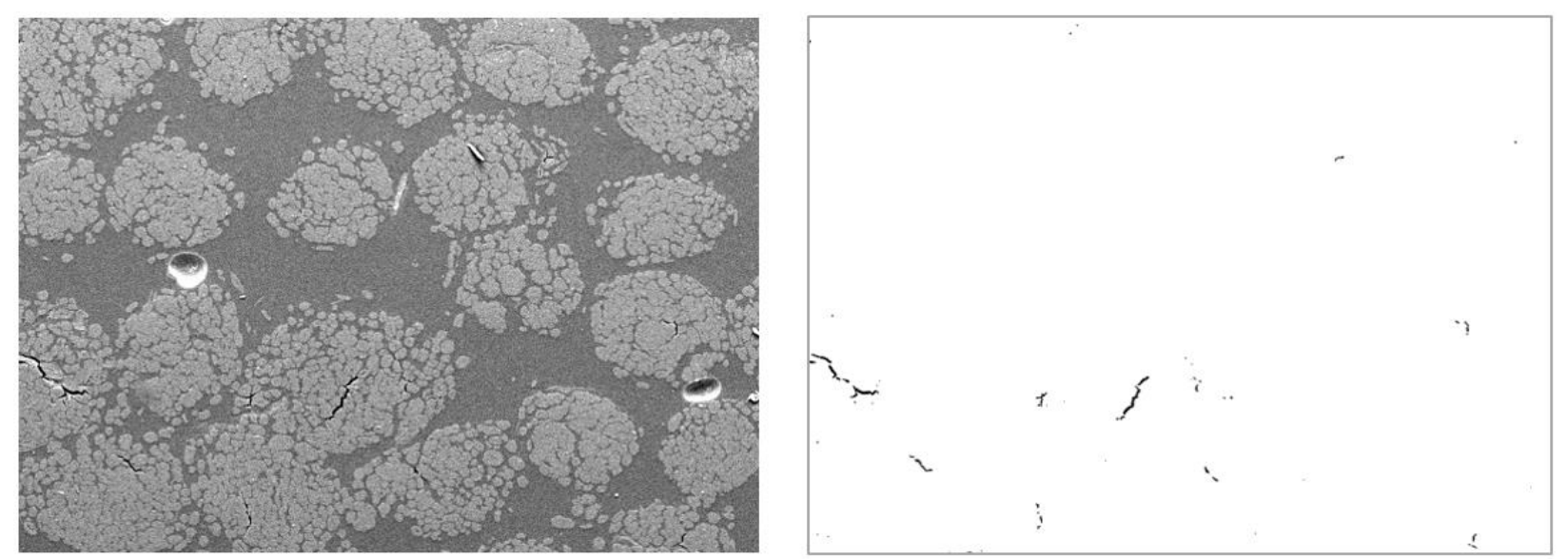

Figure 4-5: Grayscale SEM and binarized/thresholded SEM for crack isolation

\subsubsection{Derivation from Experimental Data}

The following procedure describes in detail the derivation of model parameters from experimental data. This approach was previously used by Knipprath [41] for carbon fiber binder yearn composites. Applying MDT in reverse, allows one to derive most parameters from four distinct fiber orientations: $0^{0}, 90^{\circ}, \pm 45^{\circ}$, and $67.5^{\circ}$. It should be noted that experientially derived parameters are meant for linear multi-ply models such as Classical Laminate Theory, and in this study, they served as a good initial guess for the global optimizer. Detailed sample calculations can be found in Appendix A.

\subsubsection{Fiber Direction $\left(0^{0}\right)$}

Several specimens subjected to monotonic as well as load/unload conditions were used to calculate material parameters. The initial undamaged elastic modulus is found from the initial (linear) segment of the stress/strain curve for each specimen. The actual undamaged elastic modulus is taken as the average of the specimen-specific undamaged elastic moduli:

$$
\begin{gathered}
E_{11}^{0}(n)=\left(\frac{\sigma_{11}^{\prime \prime}-\sigma_{11}^{\prime}}{\varepsilon_{11}^{\prime \prime}-\varepsilon_{11}^{\prime}}\right)_{n} \\
E_{11}^{0}=\sum_{n=1}^{n=5} \frac{E_{11}^{0}(n)}{5}
\end{gathered}
$$


The undamaged Poisson's ratio is found in three steps: 1) the Poisson's ratio is found for each load increment of each test, 2) the median Poisson's ratio of each test is calculated, 3) the overall undamaged Poisson's ratio is the average of the five means:

$$
\begin{gathered}
\text { mean } v_{12}^{0}(n)=\text { mean }\left[-\left(\frac{\varepsilon_{y}}{\varepsilon_{x}}\right)_{i}\right], \text { where } i \leq 0, i \in Z \\
v_{12}^{0}=\sum_{n=1}^{n=5} \frac{v_{12}^{0}(n)}{5}
\end{gathered}
$$

Each specimen failed at slightly different stresses, therefore, the ultimate stresses and strains are calculated as the average of the largest absolute stress or strain value recorded for each specimen:

$$
\sigma_{11}^{u}=\frac{\sum_{n=1}^{n=5} \min \left(\sigma_{11}(n)\right)}{5} \varepsilon_{11}^{u}=\frac{\sum_{n=1}^{n=5} \min \left(\varepsilon_{11}(n)\right)}{5} \varepsilon_{22}^{u}=\frac{\sum_{n=1}^{n=5} \max \left(\varepsilon_{22}(n)\right)}{5}
$$

Note that remaining parameters derived for this fiber orientation make use of data collected only from the load/unload tests. Points of interest are isolated for the upper and lower limits of the cycle seen in

Figure 3-5. Effectively, these pivot points are the locations where the slope of the stress-strain curve changes its sign. These points were used to calculated the Rise and Run of the stress-strain curve for each loop.

$$
\text { Rise }_{i}=\left(\sigma^{U}-\sigma^{L}\right)_{i} \quad \& \quad \operatorname{Run}_{i}=\left(\varepsilon^{U}-\varepsilon^{L}\right)_{i}
$$

where superscripts $U, L, D, p$, and 0 stand for Upper, Lower, Damaged, plastic, and undamaged respectfully.

Referring to Figure 3-5, the Rise and Run of each loop were used to calculate the cycle-specific elastic moduli and the apparent plastic strains:

$$
\begin{gathered}
E_{i}^{D}=\frac{\text { Rise }_{i}}{\text { Run }_{i}} \\
\varepsilon_{i}^{p}=\left(\varepsilon^{L}-\left(\frac{\sigma^{L}}{E^{D}}\right)\right)_{i}
\end{gathered}
$$

Damage in principal directions was calculated using the damaged and undamaged elastic moduli. Following which, the thermodynamic damage energy release rates were calculated: 


$$
\begin{gathered}
D_{i}=1-\frac{E_{i}^{D}}{E_{i}^{0}} \\
Y_{11}=\frac{\sigma_{11}^{2}}{2 E_{11}^{0}\left(1-D_{11}\right)^{2}}
\end{gathered}
$$

Plotting the root of the thermodynamic force $\left(\sqrt{Y_{11}}\right)$ against the cumulative damage, yields the evolution shape of damage. This is seen in Section 5.2.2.1; where data from all applicable specimens was used to determine the overall fibre-direction damage evolution in compression.

In order to determine the plasticity evolution, shear integrity $(\Omega)$ for each point of interest was calculated, which was further used to find incremental and accumulated effective equivalent plastic strains as follows:

$$
\begin{gathered}
\Omega_{i}=\left(1-D_{L}\right)_{i} \\
(\Delta p)_{i}=\Delta \varepsilon_{L}^{p}=-\frac{1}{2} \frac{\left(\varepsilon_{L_{i+1}}^{p}-\varepsilon_{L_{i}}^{p}\right)}{\Omega_{i+1}+\Omega_{i}} \\
p_{i}=p_{i-1}+(\Delta p)_{i}
\end{gathered}
$$

The experimental equivalent stress yield function points $\left(f_{i}\right)$ were calculated as follows:

$$
f_{i}=\left(R_{i}+R_{0}\right)=-\frac{\sigma_{L_{i}}}{\Omega_{i}}
$$

Next, the yield function vs accumulated plastic strain was plotted, and a power function was fitted to the data:

$$
R_{i}=\beta *\left(p_{i}\right)^{\alpha}
$$

Finally, the error between the experimental and predicted yield function points was calculated as follows:

$$
\text { error }=\frac{(\text { prediction }- \text { experimental })^{2}}{(\text { experimental })^{2}}
$$

Excel's 'Solver' function was used to reduce the total amount of error for all increments, yielding the initial guess for the material parameters $\alpha$ and $\beta$. 


\subsubsection{Transverse Direction $\left(90^{\circ}\right)$}

The transverse fiber-direction tests were performed in the same manner as the longitudinal tests, on the same number of specimens. Therefore, the transverse undamaged elastic modulus, Poisson's ratio, ultimate stresses, and ultimate strains were calculated using the same procedures as seen in the fiber-direction section.

\subsubsection{Cross-ply Orientation $\left( \pm 45^{0}\right)$}

In order to derive parameters of interest for this cross-ply fiber orientation, the coefficient $B$ [33] must first be calculated. It is important to note that the equation for the expression of $B$ contains the shear modulus $G_{12}$ as an input. Theoretically, the shear modulus should be identical in tension and compression. However, as reported by Mahboob [27, 29], there is a slight difference in their magnitude. This can be explained by considering that tensile tests are more reliable due sometimes to the premature buckling of the specimens tested in compressions, which in turn reduces the accuracy of the calculated compressive shear modulus. Therefore, the tensile shear modulus of $\pm 45^{0}$ flax composites computed by Mahboob [27, 29] was used as an input in the following calculations. Moreover, results derived with the tensile and compressive shear moduli were compared, and no visible significant differences were observed.

Note that the following calculations are for ply-level parameters.

$$
B=\left[\frac{\left(m^{2}\left(2 m^{2}-1\right)+\frac{4 m^{2} n^{2} G_{12}^{T}}{E_{22}}\left(\frac{E_{22}}{E_{11}} v_{12}+1\right)\right)}{\frac{4 m^{2} n^{2} G_{12}^{T}}{E_{22}}\left(\frac{E_{22}}{E_{11}}+2 \frac{E_{22}}{E_{11}} v_{12}+1\right)+\left(2 m^{2}-1\right)\left(m^{2}-n^{2}\right)}\right]
$$

where $m=\cos \theta \quad \& \quad n=\sin \theta$

Next, another parameter denoted as $k$ [32] was derived as follows:

$$
k=-\frac{1}{2 m n}\left(B\left(1-2 m^{2}\right)+m^{2}=-0.5\right.
$$

Points of interest were isolated as previously done for the $0^{0}$ orientation. Following which, stresses and strains in principal material coordinates for each increment are found as follows; keeping in mind that parameters $B, k, m$, and $n$ are constant for this fiber orientation [30]:

$$
\sigma_{11_{i}}=B \bar{\sigma}_{x_{i}} \quad \sigma_{22_{i}}=B \bar{\sigma}_{y_{i}} \quad \tau_{12_{i}}=k \bar{\sigma}_{x_{i}}
$$




$$
\varepsilon_{11}=m^{2} \varepsilon_{x}+n^{2} \varepsilon_{y} \quad \varepsilon_{22}=n^{2} \varepsilon_{x}+m^{2} \varepsilon_{y} \quad \varepsilon_{12}=-n m\left(\varepsilon_{x}-\varepsilon_{y}\right) \quad \gamma_{12}=2 \varepsilon_{12}
$$

Rises, runs, damaged elastic and shear moduli, and apparent plastic strains for this fiber orientation were calculated as previously seen in the $0^{0}$ section. Next, these values are used to find the principal direction damages, and the thermodynamic force conjugate of damage:

$$
\begin{gathered}
D_{11_{i}}=1-\frac{E_{11_{i}}^{D}}{E_{11}^{0}} \& \quad D_{22_{i}}=1-\frac{E_{22_{i}}^{D}}{E_{22}^{0}} \quad \& \quad D_{12_{i}}=1-\frac{G_{12_{i}}^{D}}{G_{12}^{0}} \\
Y_{12_{i}}=\frac{\tau_{11_{i}}^{2}}{2 G_{12}^{0}(\gamma)\left(1-D_{12 i}(\gamma)\right)^{2}}
\end{gathered}
$$

where $G_{12}^{0}(\gamma)$ and $D_{12}(\gamma)$ are shear modulus and shear damage calculated form $\gamma$ rather than $\varepsilon$. Plastic strain and damage is further used to calculate the thermodynamic coupling parameter $A_{t s}$ :

$$
\left(A_{t s}\right)_{i}=\frac{\left(\varepsilon_{22_{i}}^{p}-\varepsilon_{22_{i-1}}^{p}\right) \tau_{12_{i}}\left(1-D_{22_{i}}\right)^{2}}{\left(\gamma_{12_{i}}^{p}-\gamma_{12_{i-1}}^{p}\right) \sigma_{22_{i}}\left(1-D_{12_{i}}\right)^{2}}
$$

where $i$ denotes the number of the point of interest.

And the average value for $A_{t s}$ for this test was taken:

$$
A_{t s}=\frac{\sum_{i=1}^{i=5} a^{2}{ }_{i}}{5}
$$

Note that the following calculations are in regard to laminate level parameters; where stresses and strains are not undergoing transformations seen in Eqs. 4.44 and 4.45. Therefore, subscripts such as $11 \& 22$ are replaced with $L \& T$ (Longitudinal \& Transverse) to avoid confusion:

Points of interest were singled out from stress-strain data for upper and lower cycle sections. Their rises and runs were found as follows:

$$
\operatorname{Rise}_{L_{i}}=\left(\sigma_{x}{ }^{U}-\sigma_{x}{ }^{L}\right)_{i} \quad \operatorname{Run}_{L_{i}}=\left(\varepsilon_{x}{ }^{U}-\varepsilon_{x}{ }^{L}\right)_{i} \quad \operatorname{Run}_{T_{i}}=\left(\varepsilon_{y}{ }^{U}-\varepsilon_{y}{ }^{L}\right)_{i}
$$

Damaged elastic moduli, plastic strains, damage, thermodynamic damage energy release rates, shear integrity, effective equivalent plastic strain, equivalent stress yield function points, parameters $\alpha$ and $\beta$, and the error were fund as previously seen in Eqs. 4.32-4.41, but with the corresponding subscripts $L \& T$. 


\subsubsection{4 $\pm 67.5^{0}$ Orientation}

\section{Ply level:}

The same approach was used as in the calculation of the $\pm 45^{0}$ case, up to the thermodynamic force conjugates; noting that the maximum value of $\sqrt{Y_{22}}$ is $\sqrt{Y_{22}^{U}}$.

In contrast to the $\pm 45^{0}$ calculations, additional thermodynamic conjugate $Z(\varepsilon)$ was required to be calculated; which was further used to calculate the transverse-shear coupling constant $b$, and the parameter $Y_{t s}$ :

$$
\begin{gathered}
Z_{12 i}\left(\varepsilon^{e}\right)=\frac{1}{2} E_{12}^{0}\left(\operatorname{Run} \gamma_{12}\right)_{i}^{2} \text { and } Z_{22 i}\left(\varepsilon^{e}\right)=\frac{1}{2} E_{22}^{0}\left(v_{12}^{0} \operatorname{Run}_{11_{i}}+\text { Run }_{22 i}\right)^{2} \\
b_{t s_{i}}=\frac{\left(\left(D_{1 i_{i}}\left(\gamma_{12}\right) * \sqrt{Y_{12}^{c}}+\sqrt{Y_{12}^{0}}\right)^{2}-Z_{12_{i}}\left(\varepsilon^{e}\right)\right)}{Z_{22_{i}}\left(\varepsilon^{e}\right)} \\
Y_{t s}=\sqrt{Y_{12}+b_{t s} Y_{22}}
\end{gathered}
$$

Where the parameter $b$ is taken as the average value of $b_{i}$

\section{Laminate level:}

Points of interest, damaged elastic moduli, plastic strain, damage, thermodynamic force, shear integrity, plastic strain, and power law predictions were calculated similarly to the $\pm 45^{0}$ laminate section.

\subsubsection{Parameter Sensitivity Analysis}

A sensitivity analysis was conducted in order to better understand the impact of each parameter on damage and plasticity, for each type of fiber direction specimens. There are many possible approaches at performing sensitivity analysis, and they heavily depend on the nature of the problem as well as the extent of available time and effort. Hamby [45] performed an extensive comparison of sensitivity analysis techniques. It was concluded that for our case, a simple one-ata-time sensitivity measure would provide sufficient information for a relatively low cost of time and effort. The main concept of this method is to vary one parameter at a time and keep the remainder at a constant value. At the end, the sensitivity of the parameter is computed by comparing the output of the varied parameter with the output of the base case. The base case uses 
variable values derived from experimental data, and non-derivable values (coupling constants) were taken as a random guess, that provided a sufficiently good fit.

A total of fourteen variables were tested (Table 4-1) for four distinct fiber configurations, resulting in fifty-six varied cases and four base cases analyzed. Most variables were increased to a value of $360 \%$ of the base value. Few parameters identified as highly sensitive were increased to $120 \%$, they can be seen highlighted in Table 4-1.

Table 4-1: Flax/Epoxy parameters

\begin{tabular}{|c|c|c|c|c|c|c|c|c|c|c|c|c|c|c|}
\hline Number & 1 & 2 & 3 & 4 & 5 & 6 & 7 & 8 & 9 & 10 & 11 & 12 & 13 & 14 \\
\hline $\begin{array}{l}\text { Designated } \\
\text { Parameter }\end{array}$ & $Y_{f}^{0}$ & $Y_{f}^{C}$ & $\sigma_{f}^{0}$ & $\alpha_{f}$ & $\beta_{f}$ & $Y_{s}^{0}$ & $Y_{s}^{C}$ & $Y_{t}^{0}$ & $Y_{s}^{C}$ & $b$ & $A_{t s}$ & $\sigma_{t s}^{0}$ & $\alpha_{t s}$ & $\beta_{t s}$ \\
\hline
\end{tabular}

The overall outcome is visualized in Figures 4-6 through 4-9. These figures demonstrate that the fibre direction parameters are independent of shear-transverse parameters, as expected. However, shear direction is influenced by the fiber direction parameters in both damage and plasticity accumulation. Moreover, it shows the complex nature of shear-transverse coupling. This data was used to identify the crucial parameters required to be optimized for each fiber orientation, and to narrow down the boundaries of highly sensitive parameters.
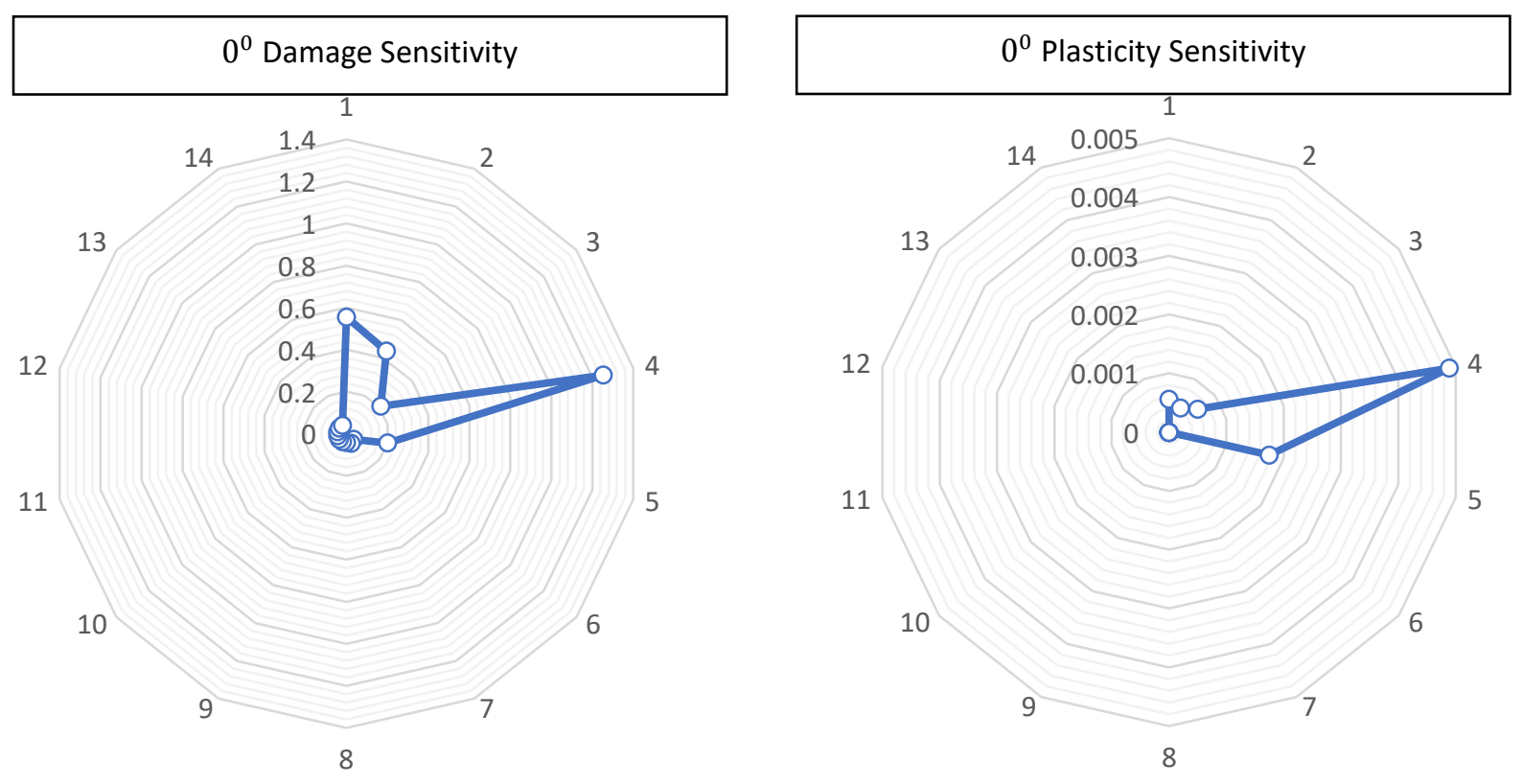

Figure 4-6: $0^{0}$ a) damage and b) plasticity sensitivity 

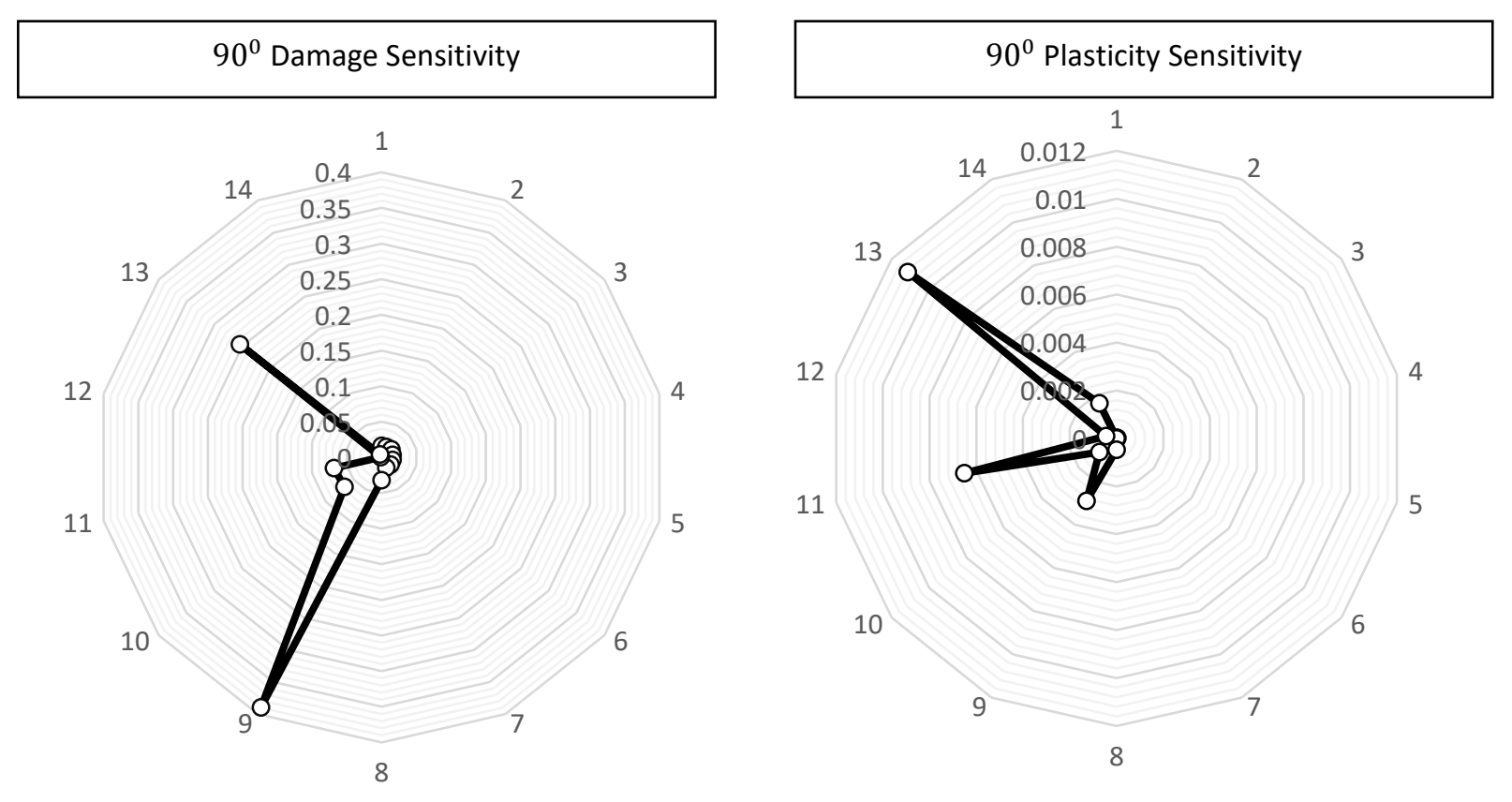

Figure 4-7: $90^{\circ}$ a) damage and b) plasticity sensitivity
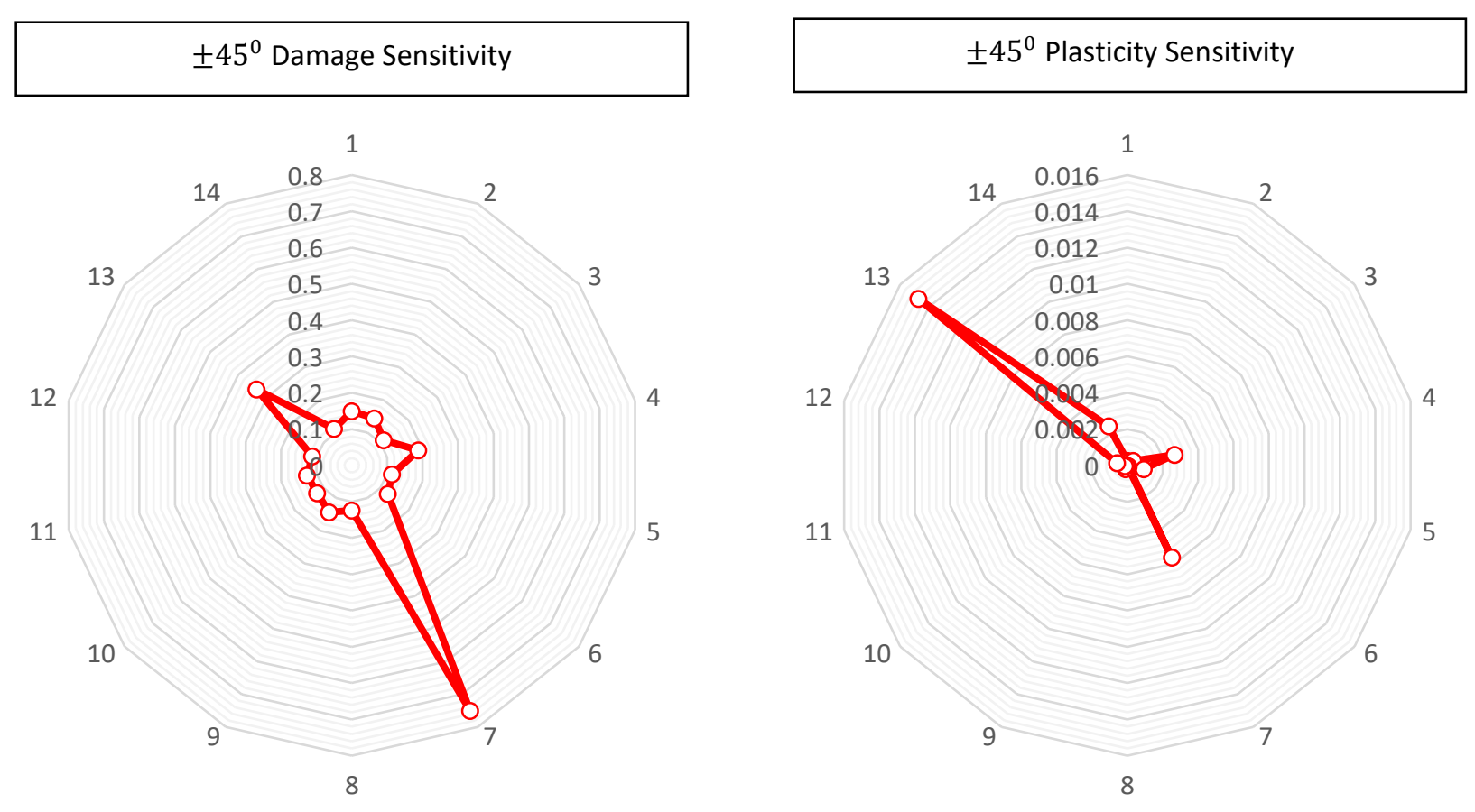

Figure 4-8: $\pm 45^{\circ}$ a) damage and b) plasticity sensitivity 

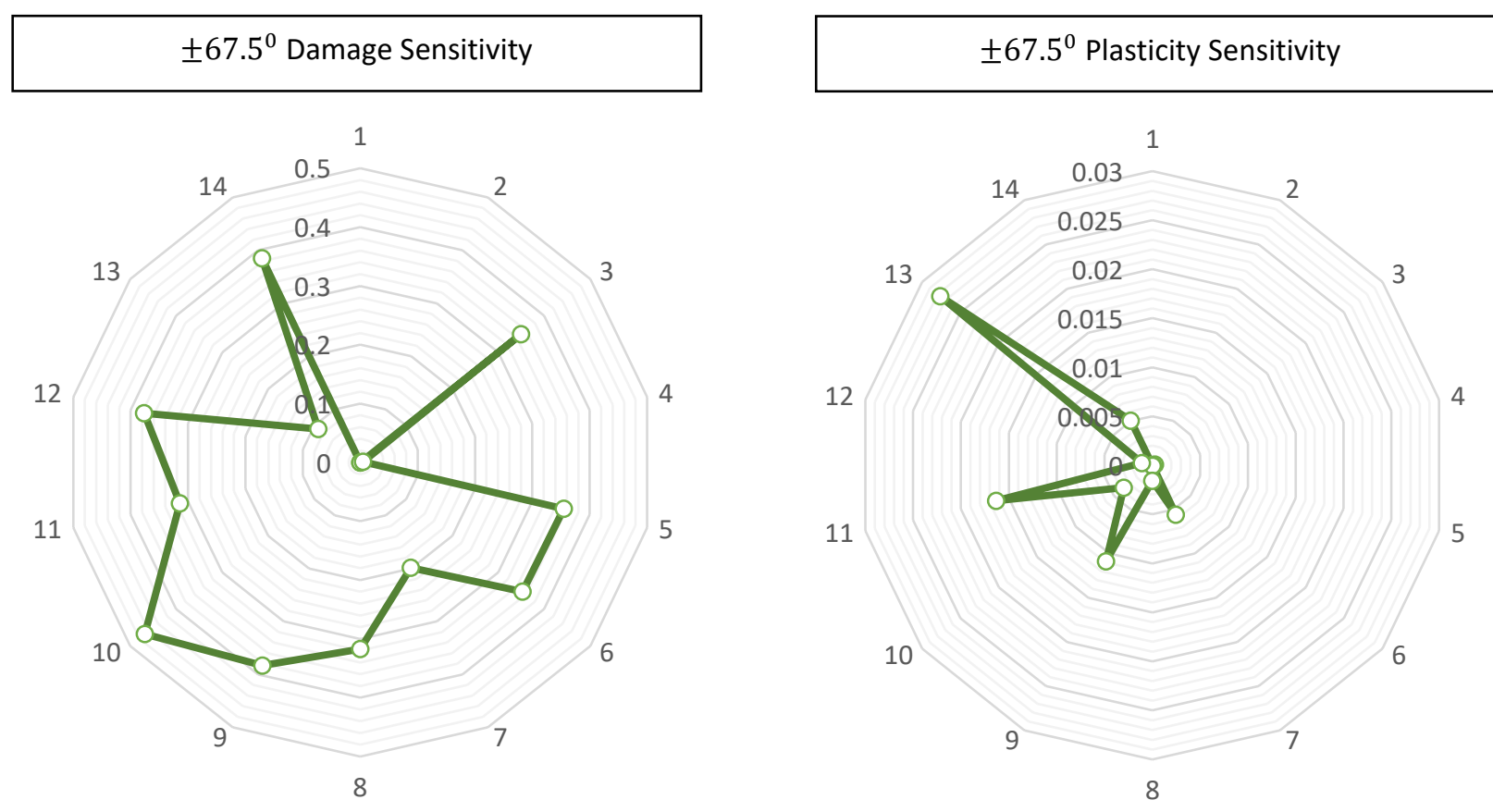

Figure 4-9: $\pm 67.5^{0}$ a) damage and b) plasticity sensitivity

\subsubsection{Parameters Optimization}

Mike McKerns, a researcher at Caltech since 2012, an author of several python packages, and a leading expert in modern optimization techniques stated: "Because of the way optimization algorithms are build, they don't always behave the same... they all have a target use case in mind, and most are customized to that case". This means that every problem has a unique optimizer that best suits it. Since the goal of this thesis was not to develop a new optimizing algorithm, the next best thing was to find an algorithm built for similar problems, and adopt it to this sty case. Several types of optimizer were explored, from simple gradient descend, to complex genetic evolution types. Knowing that a global and non-linear optimizer is required to solve the problem at hand, the selection was narrowed down to two open-source algorithms: Basin Hopping and Differential Evolution from scipy.optimize libraries [42]. As mentioned by McKerns, most problems require one to try several types of optimizers in order to zero-in on the most effective one. If the cost function is imagined as a 2D plane with elevated and descended features, such as any 3D map of a geographical region, then Basin Hopping works by taking an initial guess as the starting point and randomly jumping to different areas of the cost-function plane and evaluates the function's value at those points; gradually converging on the location with the lowest value. This optimizer 
experienced issues because several parameter combinations caused the iteration steps described in Section 4.2.2 to diverge and the optimizer would crash. An analogous to this is imagining that the 2D cost-function plane is full cracks and voids. Not being able to predict all the parameter combinations that lead to divergence, meant that one could not make the optimizer omit the problematic areas. This lead to the conclusion that Basin Hoping was an ineffective algorithm for this case.

Differential Evolution is similar to other types of genetic algorithms. It takes bounds as an input, and generates a series of possible parameter combinations that are within the bounds. This can be imagined as scattering a bunch of probes randomly distributed on the 2D cost-function plane. The probes with the lowest cost-function values would be used as parents for the new generation of probes. Here, the algorithm would mix the traits (parameters) of the most successful probes in order to achieve a new generation of probes containing the best desired qualities of the previous generation. The most promising of the new generation would get selected to become the parents of the next generation. This process would continue until the optimizer would converge on the best possible combination of parameters. For the problem at hand, this approach was found to be very effective, since strict parameter bounds were imposed on the optimizer, ensuring that it would avoid problematic combinations.

Since the model output and the experimental result for each specimen contained the same amount of discrete points, the following formula was used as a simple cost function (C.F.), minimizing the difference between the experimental and predicted strains for a given value of stress:

$$
C . F .=\sum_{i=1}^{i=m}\left[a *\left(\sum_{i=1}^{i=n}\left|\varepsilon_{L}^{\text {exp }}-\varepsilon_{L}^{\text {model }}\right|\right)+(1-a) *\left(\sum_{i=1}^{i=n}\left|\varepsilon_{T}^{\text {exp }}-\varepsilon_{T}^{\text {model }}\right|\right)\right]
$$

where $m$ is the number of specimens of different orientations, $n$ is the number of discrete stress increments per specimen, and $a$ is a weight factor giving priority to a better fit in the longitudinal direction.

Again, it is worth to note that the parameters identified in the prior sections are best suited for Classical Laminate Theory applications. Periodic Homogenization heavily relies on iterative techniques, therefore, an iterative parameter identification technique (such as this type of 
optimization) is better suited for this particular case. Nonetheless, parameters identified from experimental data were used as a good initial guess for the optimizer's parameter bounds.

\subsection{MMDM Implementation}

This section will describe the methodology used in the numerical implementation of the Modified Mesoscale Damage Model. Firstly, damage and plasticity equations introduced in the Section 4.1 are used on each ply to calculate the local strain state for a given stress increment. Secondly, SMART+ algorithms [43] use iterative techniques to homogenize the strain level of each ply, resulting in the overall composite strain state prediction.

\subsubsection{Single-Ply}

An element at a certain stress state could be experiencing damage, plasticity, both, or none. Referring to Figure 4-10, suppose that one would like to calculate the strain state of a singly ply for a load increment $\Delta \sigma$. Firstly, the program checks for onset of damage by computing the thermodynamic forces described in Section 4.1.5 ( $D>0$ ?). If no damage is found to, the elastic modulus of set increment remains equal to the modulus of the previous increment $\left(E_{n}=E_{n-1}\right)$. If damage is found, then the elastic modulus is depredated using the calculated damage $\left(E_{n}=\right.$ $\left.E_{n-1}(1-D)\right)$. The elastic portion of strain is calculated $\left(\Delta \varepsilon^{e}=E_{n}^{-1} \Delta \sigma\right)$. Next, the equivalent applied stress is calculated, and onset of plasticity is checked ( $\tilde{\sigma}^{e q} \leq R_{0}$ ?). If no plasticity is found, the plastic portion of strain is zero $\left(\Delta \varepsilon^{P}=0\right)$. If plasticity is found, then the plastic strain is found using the accumulated plastic strain $(\Delta \tilde{p})$. Finally, the total strain increment is the summation of the plastic and elastic strains $\left(\Delta \varepsilon=\Delta \varepsilon^{e}+\Delta \varepsilon^{p}\right)$. 


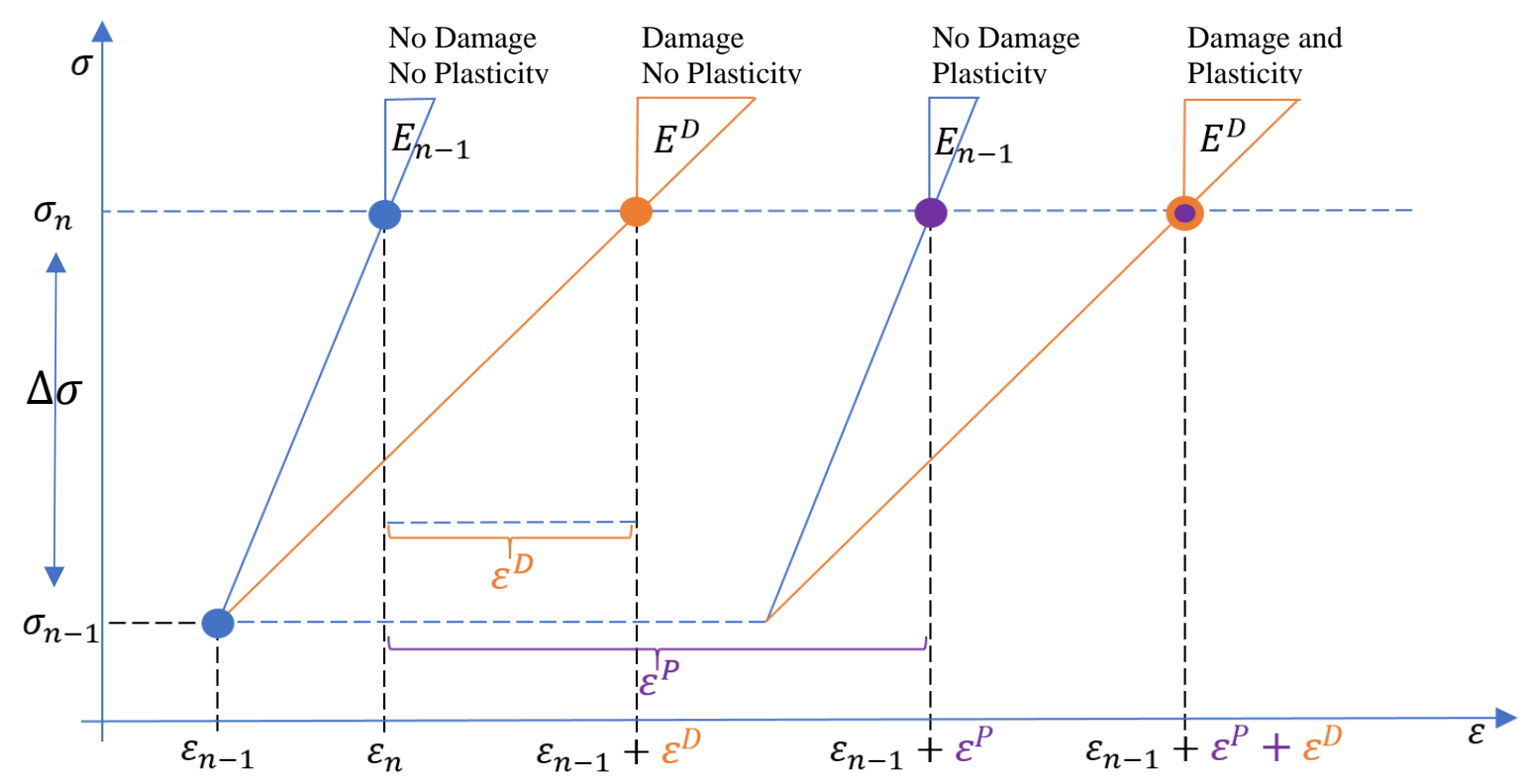

Figure 4-10: Element strain states

\subsubsection{Multi-Ply}

Load-bearing laminates are generally composed of multiple plies of similar, different, or periodically repeating orientations. Laminate theory is generally used to describe the behaviour of multiply laminates, however this theory has drawbacks, especially in predicting non-linear response of composites [29]. Flax composites exhibit irrecoverable strains, stiffness degradation, and damage evolution; moreover, internal stresses arise due to irreversible strain mismatch inbetween the plies [29]. Therefore, a new iterative approach was applied to the multi-ply model.

Periodic Homogenization used by Mahboob et al. [29] for tensile modeling of Flax composites is an advanced technique pioneered by Bensoussan et al. [44]. It allows loading in 3D and has been successfully implemented in modeling non-linearly composite response of shapememory alloys [45]. MMDT was integrated into the incrementally applied periodic homogenization material solver using open-source SAMRT+ (Smart Materials Algorithms and Research Tools [43]) scientific libraries developed by several collaborating institutions. These libraries were specifically designed to analyze and model heterogeneous materials, and the MMDM implementation was similar to previous implementations of plasticity and viscoplasticity models seen in [45]. A system of five non-linear equations (two plasticity conditions from yield 
functions $\Phi_{f}^{p} \& \Phi_{t s}^{p}$, and three related to damage functions $\Phi_{D_{11}}, \Phi_{D_{22}}, \& \Phi_{D_{12}}$ ) are mathematically treated as presented in [45].

The working principle behind multi-ply modeling is illustrated in Figure 4-11. Calculating strain states as presented in Section 3.2.1 requires one to know the exact stress state of each ply. This is unknown, since the software input is the total composite stress. Therefore, the total composite stress is decomposed it into ply-specific stresses using a material solver. Next, plyspecific analysis is performed as described in Section 3.2.1; however, there is a chance of strain mismatch due to an inaccurate initial stress decomposition. Therefore, the resulting strains are returned to the SMART+ periodic homogenization algorithms which use an iterative approach to re-adjust the ply-specific stress increments until the resulting ply strains are equivalent.

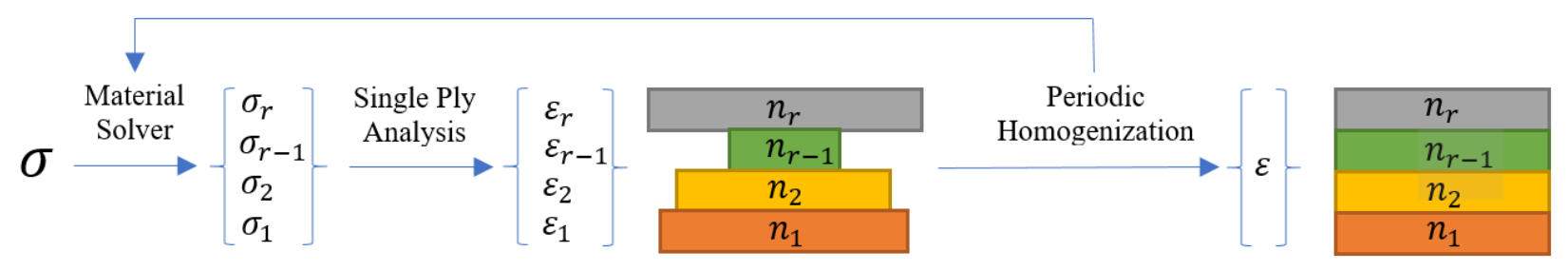

Figure 4-11: Multi-ply modeling schematic

The overall modeling process can be interpreted as a dual-stage iterative procedure. The first stage deals with individual plies, iterating the damage and plasticity states of each ply. The second stage is at the laminate level, where iterations are performed on ply-specific stress components in order to achieve a uniform strain state within the composite. A schematic representation of the whole iterative process including all the calculation steps is shown in Figure 4-12. 
1. Stress increment is selected

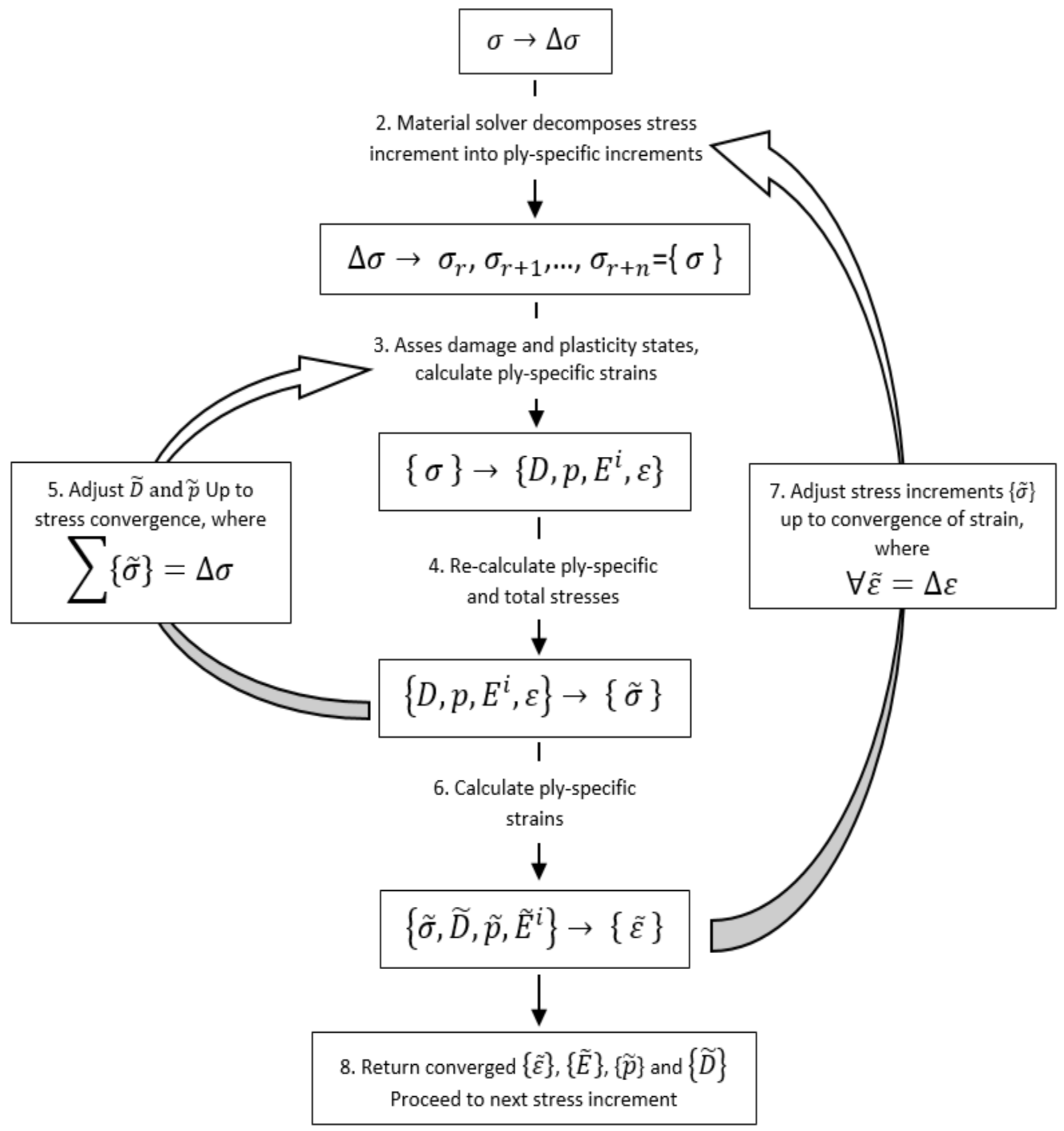

Figure 4-12: MMDM implementation schematic 


\section{Chapter 5: Model Validation and Result}

\subsection{Model Validation on E-Glass Laminates}

In this thesis, flax composites are iontroduced as an alternative to glass-reinfoced composites. Therefore, compressive validation was performed on E-glass/Polyester composites of various fiber orientations (Figure 5-1). To furher validate the versatility of the model, the MMDM was also applied to E-glass/Epoxy composites in tension with various fiber oreitations (Figure 5-2). Experimental data on the material response was extracted from the works of Amijima and Adachi [46]. Under both tensile and compressive loads, it was assumed that damage evolution was linear (even if damage itself was infintesimally small), and that all plastic evolution followed a power law simmilarly to the original formaulations of Ladeveze and Le Dantec [34]. It was observed that that the model (solid black line) succesfuly predicted the E-glass composite mechanical response to tensile and compressive loads, even at strains as high as $3 \%$.

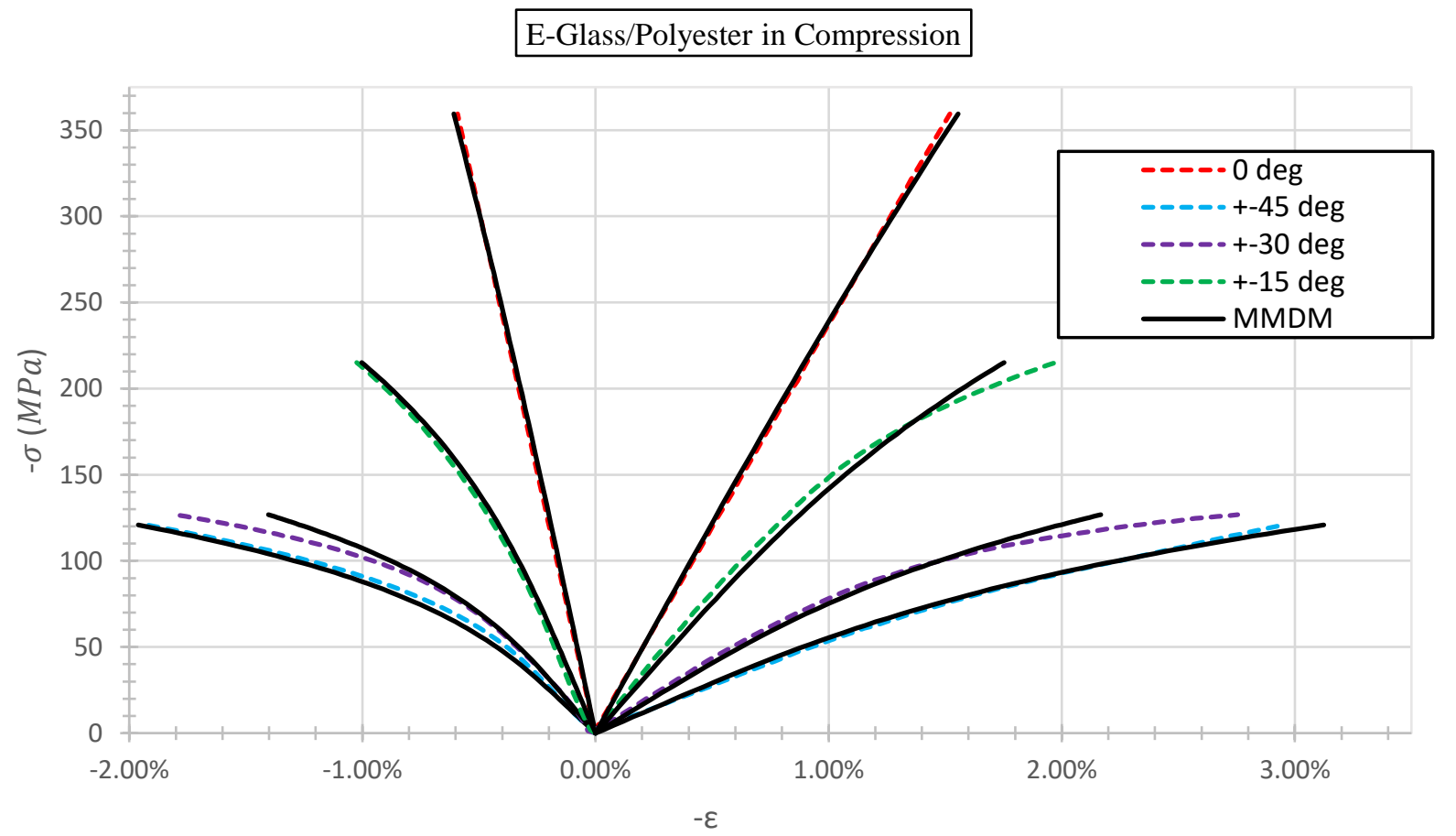

Figure 5-1: E-glass/Polyester composite compressive MMDM prediction vs experimental response [52] 


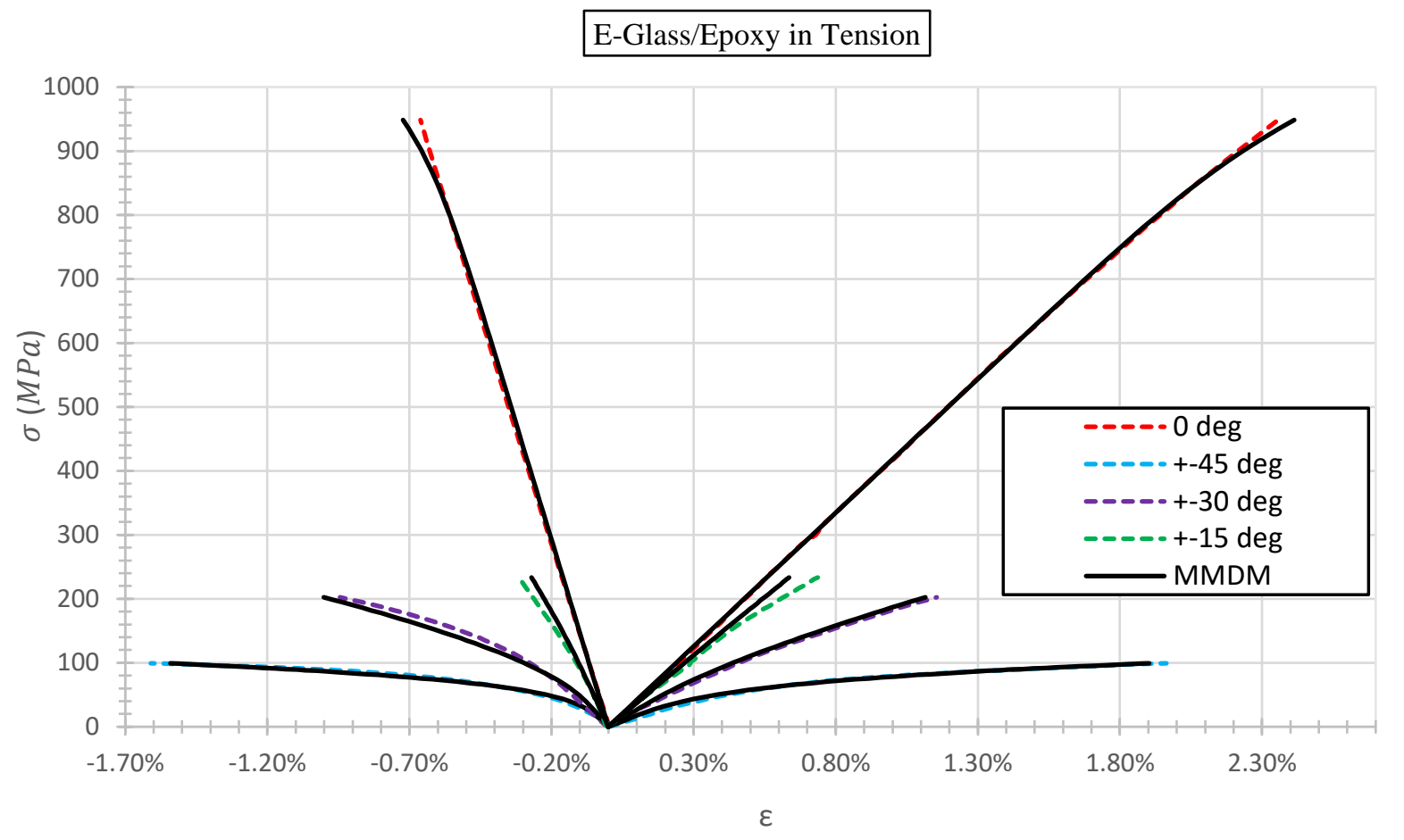

Figure 5-2: E-glass/Epoxy composite tensile MMDM prediction vs experimental response [52]

\subsection{Flax Composite Characteristics}

\subsubsection{Stress-Strain Behaviour}

From the previous works [32], it was known that Flax composites share similarities in their mechanical characteristics and behaviour under tensile and compressive loads. This phenomenon is seen in Figures 5.3 and 5.4, where red is tensile (reprinted with permission from [29]) and purple is compressive material response. Noting that the compressive response was reflected with respect to the $\mathrm{x}$ axis, in order to better visualize the similarities and differences in between tensile and compressive response.

It is noticeable that the magnitudes of the stress-strain plots are different, but the overall shape of the curves are similar. Since the theory has been shown to be effective in tensile cases, the stressstrain similarity implies that it should be able to capture the compressive behaviour as well. In accordance with the observations of Bos \& Donald as well as other authors [20, 22, 27], it is seen that the $0^{0}$ fiber-oriented samples have a greater tensile than compressive strength. Here, its observed that Flax/Epoxy composites in compression obtain the same level of damage and 
plasticity as the composite in tension at half the stress applied. Even though the average tensile undamaged elastic modulus is larger than the compressive one $(31.42 \mathrm{GPa}$ and $30.32 \mathrm{GPa}$ respectfully) they fall within the same range of value considering their respective standard deviations. The ultimate tensile strength is twice as large as the compressive one, with values of 286.7 MPa and 127.1 MPa respectfully. Epoxy-only specimens have been tested to have tensile and compressive elastic moduli of 3.03 and $3.57 \mathrm{GPa}$ respectfully, while the ultimate tensile and compressive strengths were 67.17 and $73.99 \mathrm{MPa}$ respectfully. This clearly indicates that the addition of Flax fiber oriented parallel to the load-axis, creates a composite with greatly increased elastic moduli as well as the ultimate strengths in tension and compression compared to neat epoxy.

For transverse-oriented fibers, the undamaged elastic moduli have similar values (considering measured standard deviation $\sim 5.6 \mathrm{GPa}$ ), while the ultimate compressive strength is double that of the tensile (79.94 $\mathrm{MPa}$ and $33.86 \mathrm{MPa})$. The presence of transverse fibers in epoxy nearly doubles the elastic moduli, while the tensile strength was reduced in half and the compressive strength remained the same. This is a consequence of dissimilar tensile and compressive damage mechanisms, further discussed in the next section. An interesting observation is that in the transverse direction, $90^{\circ}$ fiber-oriented samples under tension and compression experience close to no damage and plasticity.

The $\pm 45^{0}$ reinforced specimens have a slightly larger average measured shear modulus in tension than in compression (2.07 GPa and $1.63 \mathrm{GPa}$ ); however, considering the standard deviations, the difference becomes negligible. The tensile shear strength was found to be slightly smaller than the compressive one (37.35 $\mathrm{MPa}$ and 43.24 $\mathrm{MPa}$ ). The ultimate tensile and compressive strengths along the load-axis were measured to be $74.28 \mathrm{MPa}$ and $86.47 \mathrm{MPa}$, indicating that the damage mechanisms are different in tension and compression. There is notably more damage occurring under compression, as the stress-strain curve is much less linear than in tension.

Lastly, similar trends as in $90^{\circ}$ samples have been observed in the $\pm 67.5^{\circ}$, with slightly higher strength and stiffness values. This is due to the fact that the fiber component parallel to the load axis is able to carry some additional load when compared to the transverse-only case. 

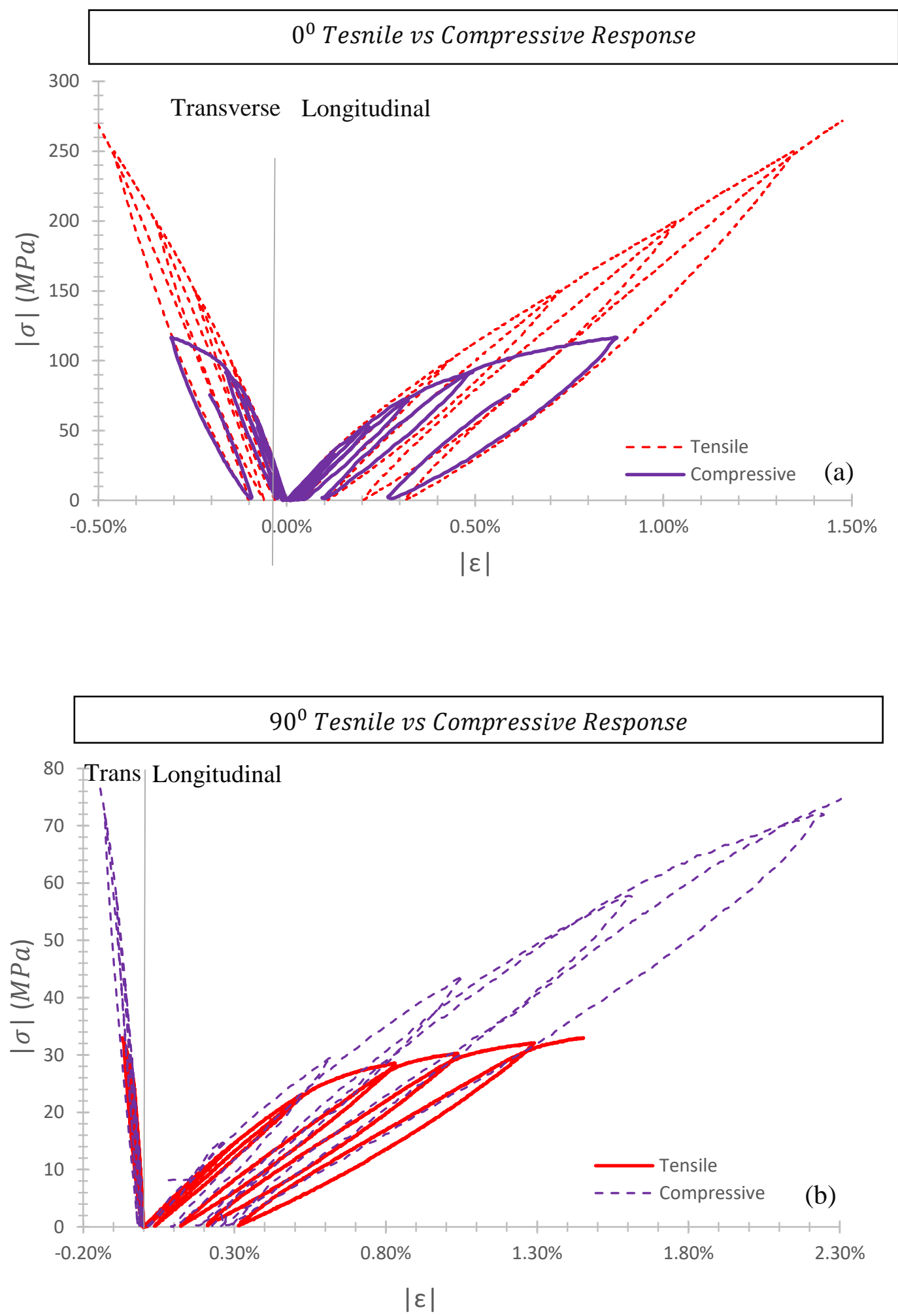

Figure 5-3: Tensile and compressive UD Flax/Epoxy mechanical response from experimental data 

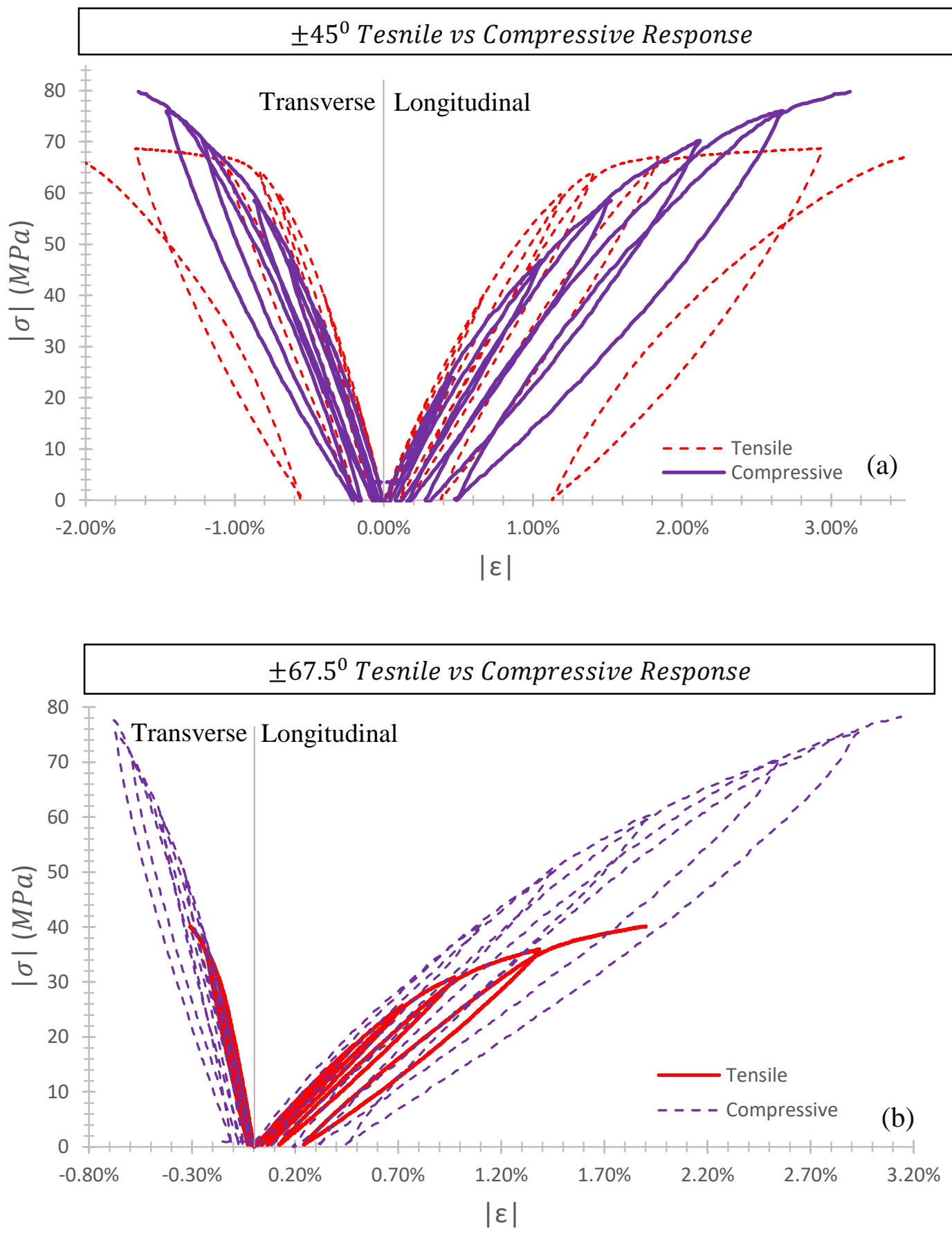

Figure 5-4: Tensile and compressive cross-ply Flax/Epoxy mechanical response from experimental data 


\subsubsection{Flax/Epoxy Damage and Plasticity}

In this section, observed damage and plasticity compressive trends of Flax/Epoxy laminates are discussed. These observations were further used in the MMDM damage and plasticity constitutive equation formulations.

\subsubsection{1 $\quad 0^{0}$ Fiber-Oriented Flax/Epoxy}

There was a total of 7 tests performed on samples of this fiber orientation, 2 monotonic and 5 of load-unload type. Applying the MDT in reverse as describe in [33], allows one to expose the composite's damage and plasticity evolution trends. The fiber-direction damage force $\left(Y_{f}\right)$ was plotted versus the accumulated fiber direction damage $\left(D_{11}\right)$ as seen in Figure 5-5a. The compressive damage evolution of flax composites with $0^{0}$-oriented fibers is identified as generally-linear. Some deviation from the trend is associated with the specimen's onset of buckling; which is not of concern in this thesis. Figure 5-5b shows the composite's compressive plasticity evolution trend, which follows a power-shape function with a positive fractional exponent.
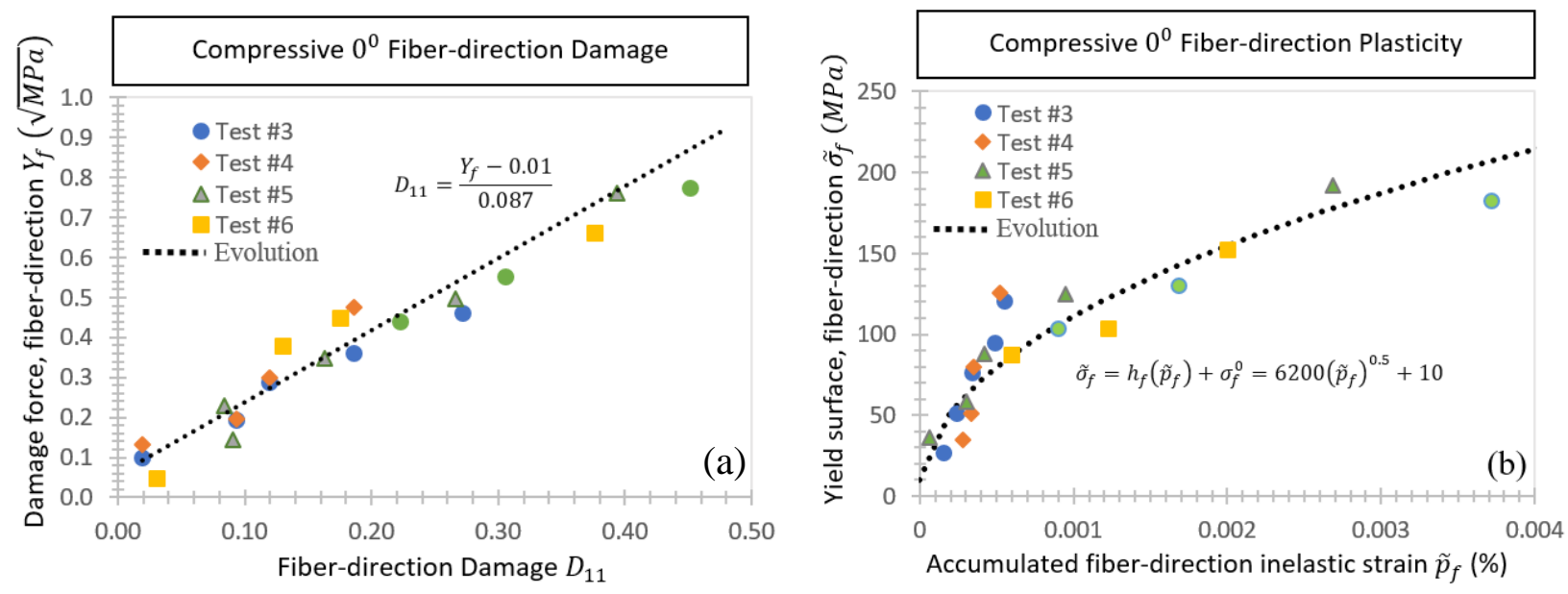

Figure 5-5: Fiber-direction a) Damage and b) Plasticity evolution

\subsubsection{2 $\pm 45^{0}$ and $\pm 67.5^{0}$ Fiber-Oriented Flax/Epoxy}

The same methodology as seen in the previous section was applied to samples with fiber directions other than $0^{0}$. Noting that the damage $\left(D_{L}\right)$ and plasticity $\left(\tilde{p}_{L}\right)$ trends observed are in reference to the load axis. Under compressive loads, $\pm 45^{0}$ and $\pm 67.5^{\circ}$ fiber-oriented specimens follow linear damage evolutions and power-shaped plasticity evolutions as seen in Figure 5-6 and Figure 5-7. 

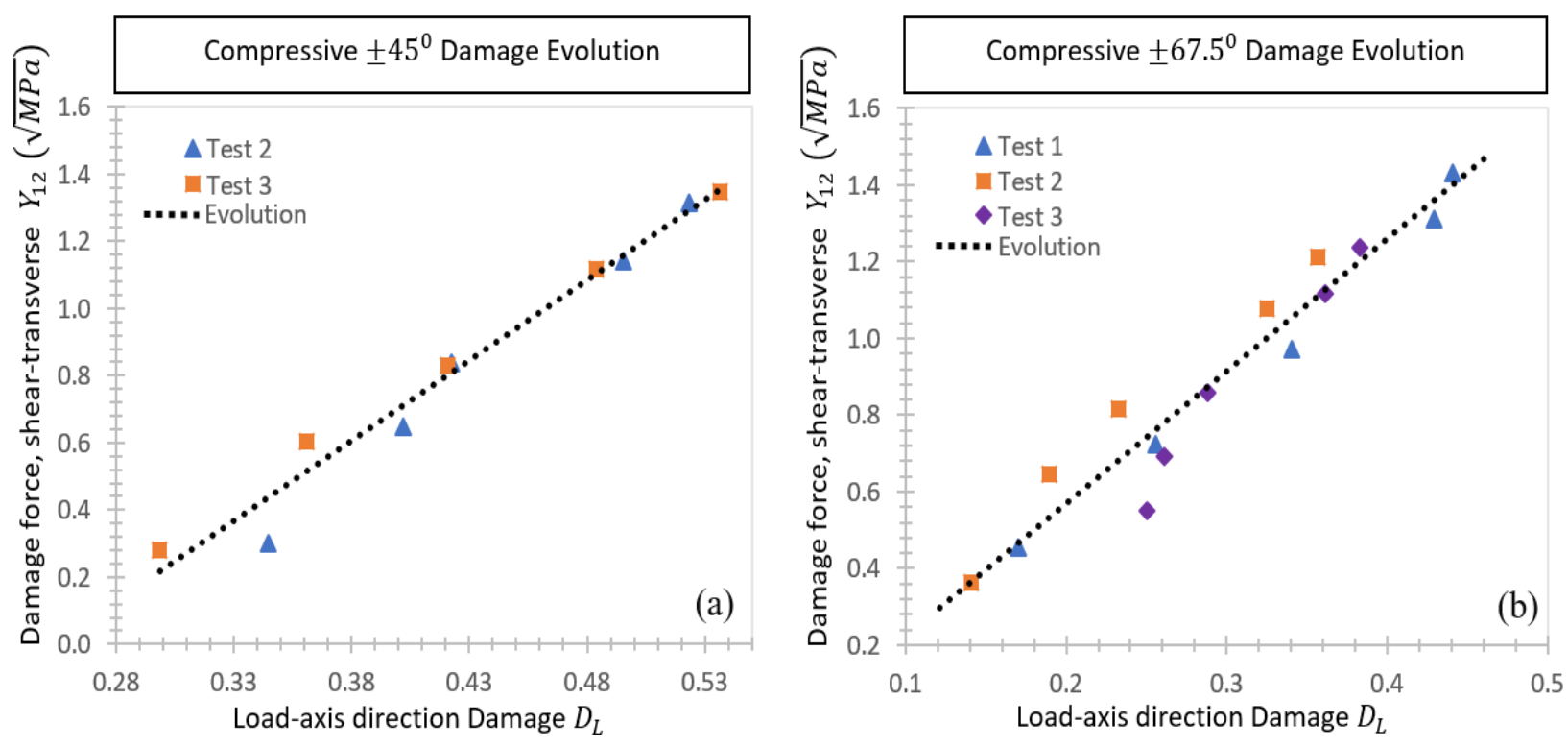

Figure 5-6: Compressive shear-direction damage evolution: a) $\pm 45^{0}$, b) $\pm 67.5^{0}$
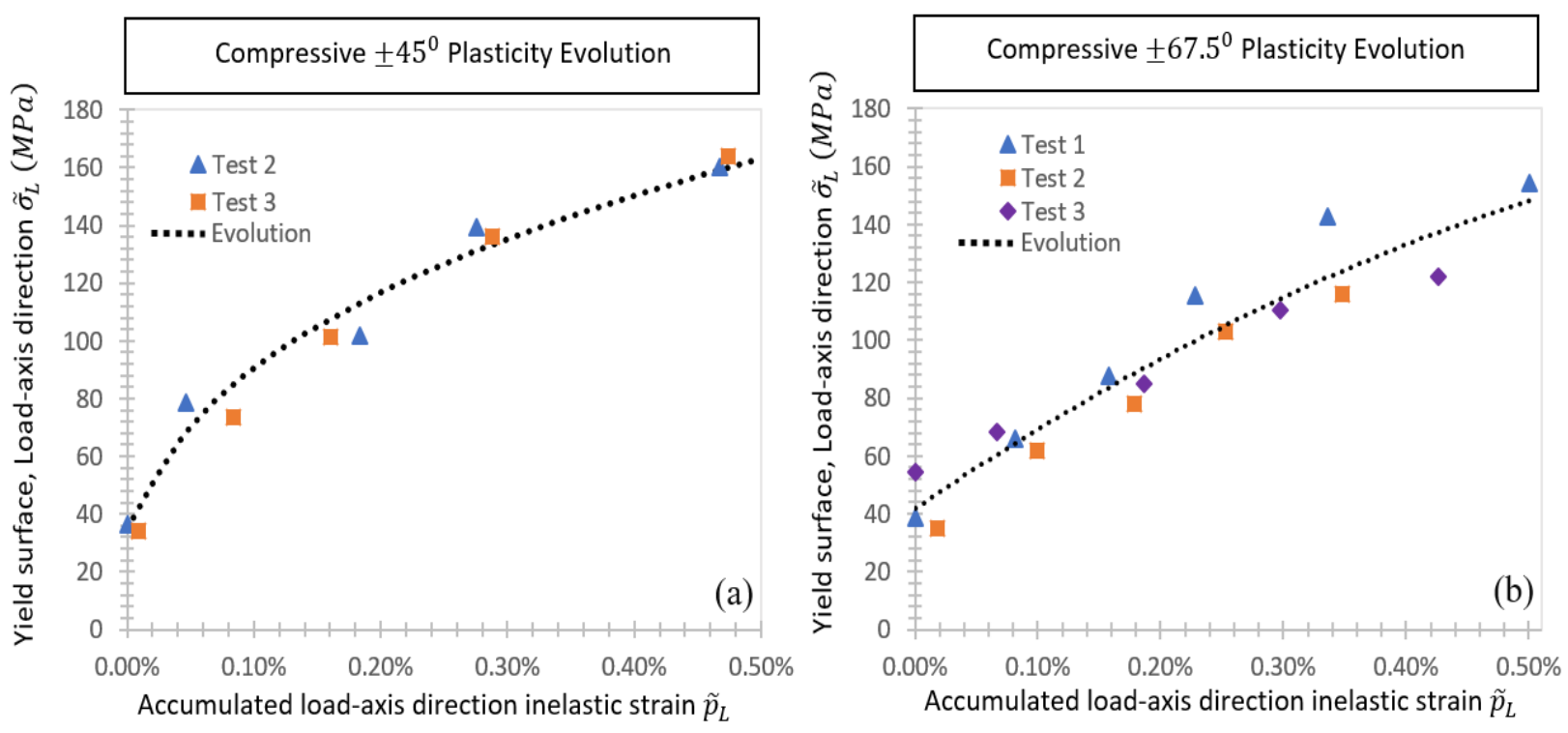

Figure 5-7: Compressive shear-direction plasticity evolution: a) $\pm 45^{0}$, b) $\pm 67.5^{0}$

\subsubsection{3 $90^{0}$ Fiber-Oriented Flax/Epoxy}

$90^{0}$ fiber-oriented samples exhibited exponential trends for both damage and plasticity evolutions (Figure 5-8). This raises a substantial problem, due to MDT fundamental formulation of linear shear-transverse coupling, the theory intrinsically requires the transverse direction damage evolution to be linear and the plasticity evolution to be power-shaped. Fortunately, a linear 
fit for damage evolution and a power-shaped plasticity evolution with a very small curvature were found to fit sufficiently well as seen in Figure 5-9.
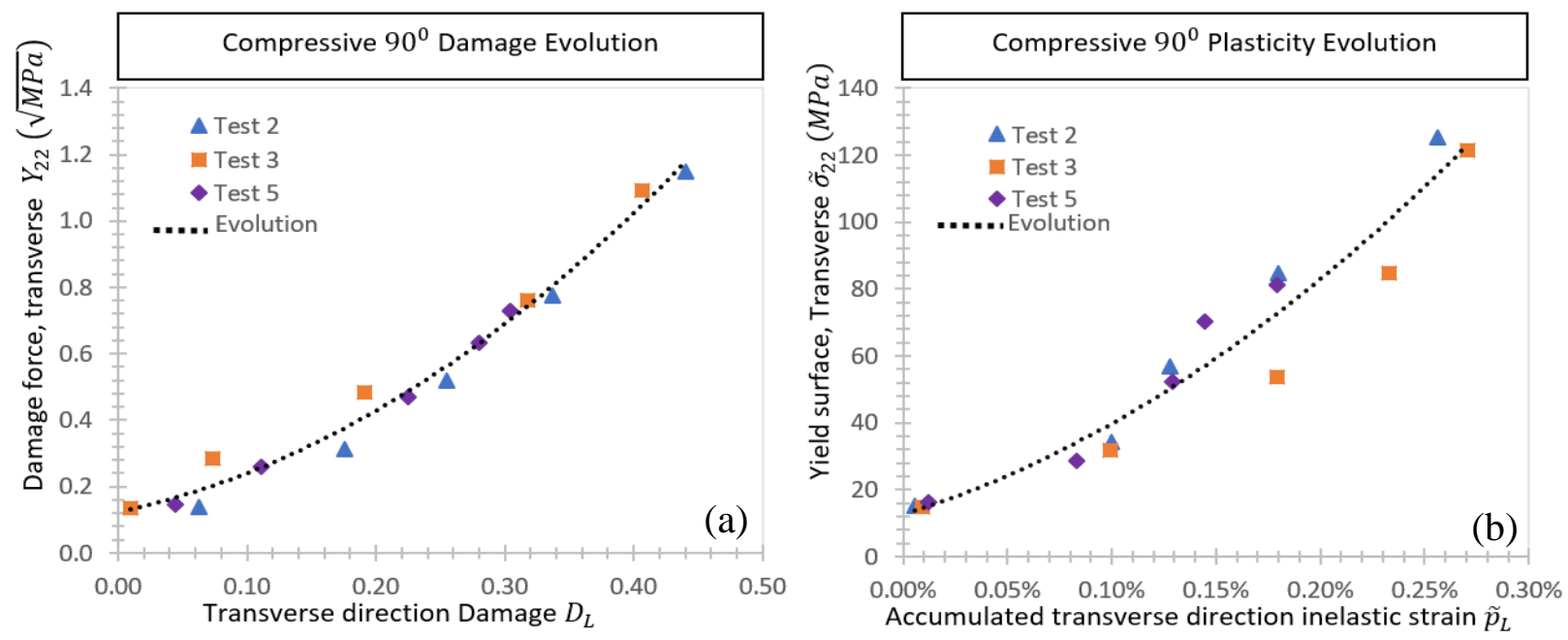

Figure 5-8: Transverse exponential a) damage evolution, b) plasticity evolution
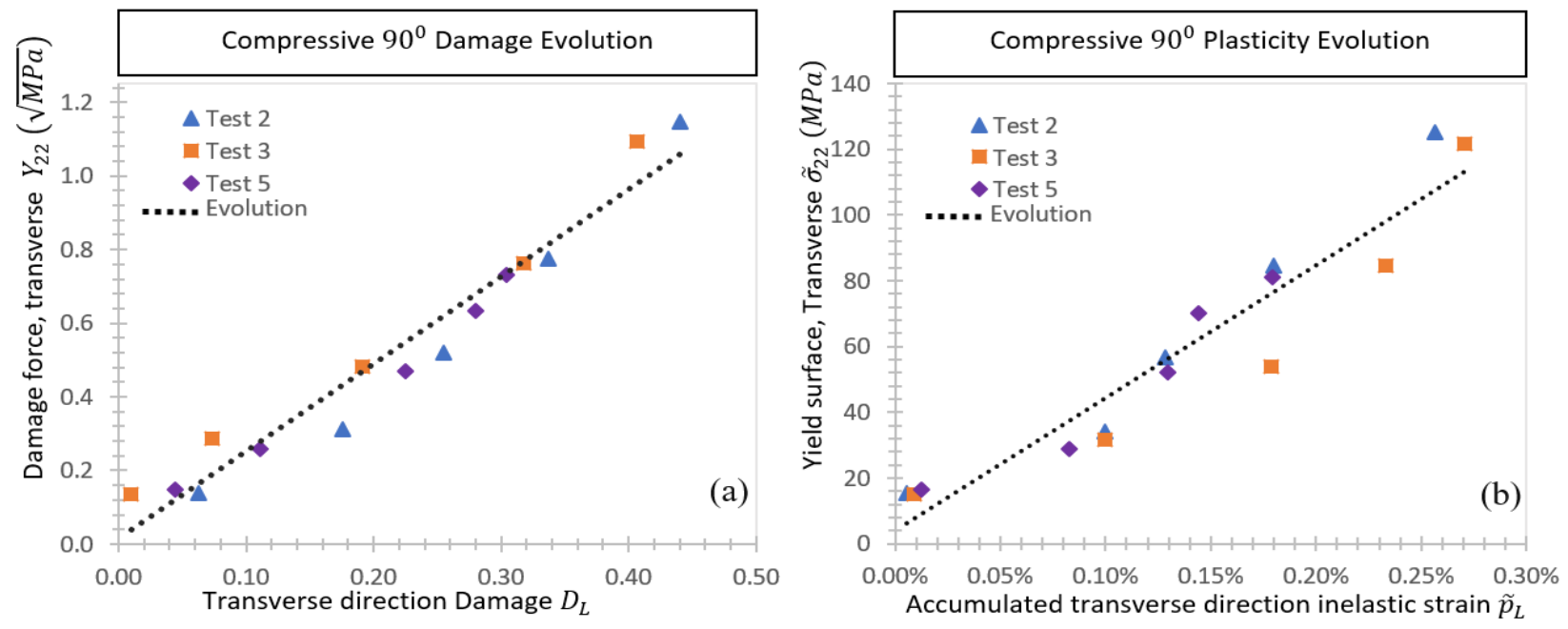

Figure 5-9: Transverse a) linear damage evolution, b) gradual power plasticity evolution 


\subsection{Flax/Epoxy Compressive Response and Simulation}

In this study, 4 principal ply orientations were used to identify Flax/Epoxy compressive parameters via the optimization methods discussed in Chapter 4. At least four monotonic and/or multiple-cycled progressive loading tests were performed on each fiber orientation and used in parameter identification; thus, the identified parameters are listed in Table 5-1. The simulated results can be seen in Figures 5-10 through 5-13, noting that the saw-tooth shaped load-path shows the cyclical and progressively increasing load applied to the samples. It is easily noticeable that the model excellently predicts both damage and plasticity evolution of $0^{0}$ and $\pm 67.5^{\circ}$ orientations. In the case of $90^{\circ}$, the model is capable of predicting the overall strain state of the material, but cannot decompose it accurately into its elastic and plastic parts. This is an outcome of the fundamental difference in the transverse-fiber specimen plasticity evolution. As previously mentioned in Section 5.2.1, transverse samples experienced exponential plasticity evolution, while all others experienced a power-law with a fractional exponent (between 0 and 1). The current shear-transverse coupling constant $b$ cannot transform the power plasticity evolution curve into an exponential one for the transverse direction. This however, is not a major concern since there are limited scenarios where fiber reinforcement is placed perpendicular to the load-axis. Moreover, if this case is encountered, the model is capable of predicting the overall strain state of transverseoriented composite. Lastly, for the $\pm 45^{\circ}$ orientation, it is observed that the model does an excellent job at predicting the material strain state in the longitudinal direction, but cannot match the transverse experimental result. There are several possible reasons for this mismatch, due to this fiber orientation's susceptibility to delamination, ply reorientation, and buckling. Nonetheless, the model has shown that its overall capable to predict flax-epoxy strain states of various fiber orientations.

Lastly, it should be noted that the compressive model was fitted to the material response of a single sample that exhibited an average material response in comparison to other tested samples. Due to the nature of composite materials and the manufacturing procedures, there is a larger deviation in material properties compared to typical metals. Therefore, one should keep in mind that some deviation between a specific sample behaviour and the average material behaviour should be expected. 
Table 5-1: Flax/Epoxy compressive-specific MMDM parameters

\begin{tabular}{lccccc}
\hline Material Property & $\boldsymbol{E}_{\mathbf{1 1}}^{\mathbf{0}}(\mathrm{GPa})$ & $\boldsymbol{v}_{\mathbf{1 2}}^{\mathbf{0}}$ & $\boldsymbol{E}_{\mathbf{2 2}}^{\mathbf{0}}(\mathrm{GPa})$ & $\boldsymbol{v}_{\mathbf{2 1}}^{\mathbf{0}}$ & $\boldsymbol{G}_{\mathbf{1 2}}^{\mathbf{0}}(\mathrm{GPa})$ \\
\hline Fiber Direction & 32 & 0.087 & 5.23 & 0.396 & 1.66 \\
\hline & $Y_{f}^{0}(\sqrt{\mathrm{MPa}})$ & $Y_{f}^{C}$ & $\sigma_{f}^{0}(\mathrm{MPa})$ & $\alpha_{f}$ & $\beta_{f}$ \\
\hline Shear Damage & 0.01 & 1.64 & 5.653 & 0.445 & 2998 \\
\hline $\begin{array}{l}Y_{12}^{\max } \\
(\mathrm{MPa})\end{array}$ & $Y_{s}^{0}(\sqrt{\mathrm{MPa}})$ & $Y_{s}^{c}(\sqrt{\mathrm{MPa}})$ & & \\
\hline $\begin{array}{l}\text { Transverse } \\
\text { coupled damage }\end{array}$ & 1.26 & 0.001 & 2.32 & & \\
\hline $\begin{array}{l}\text { Transverse-Shear } \\
\text { yield \& } \\
\text { inelasticity }\end{array}$ & $A_{t s}(\mathrm{MPa})$ & $Y_{22}^{\max }(\mathrm{MPa})$ & $Y_{t}^{0}(\sqrt{\mathrm{MPa}})$ & $Y_{t}^{C}(\sqrt{\mathrm{MPa}})$ & \\
\hline & & 5.03 & 0.0128 & 2.65 \\
\end{tabular}

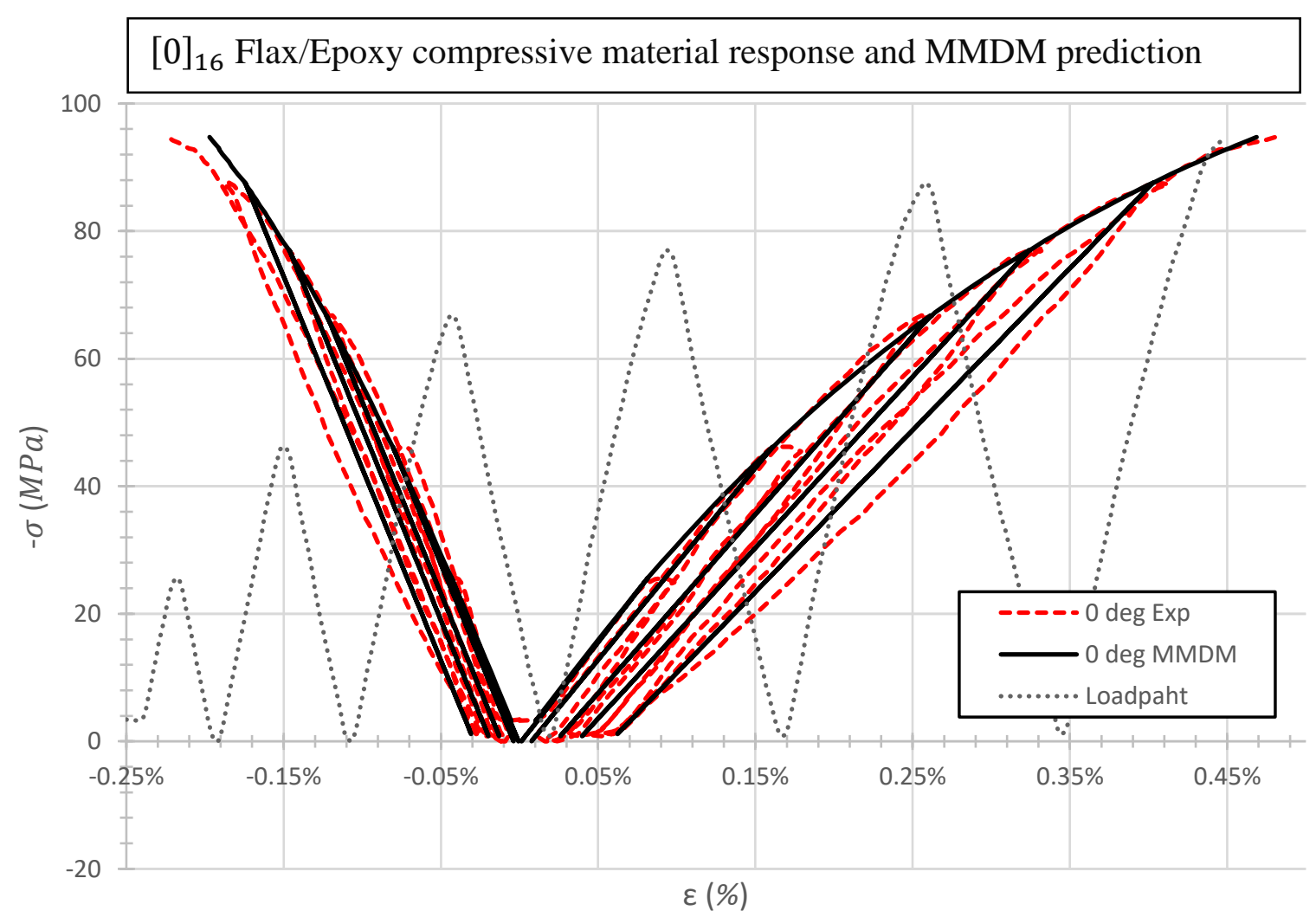

Figure 5-10: $0^{0}$ fiber-oriented Flax/Epoxy experimental behaviour and MMDM prediction 


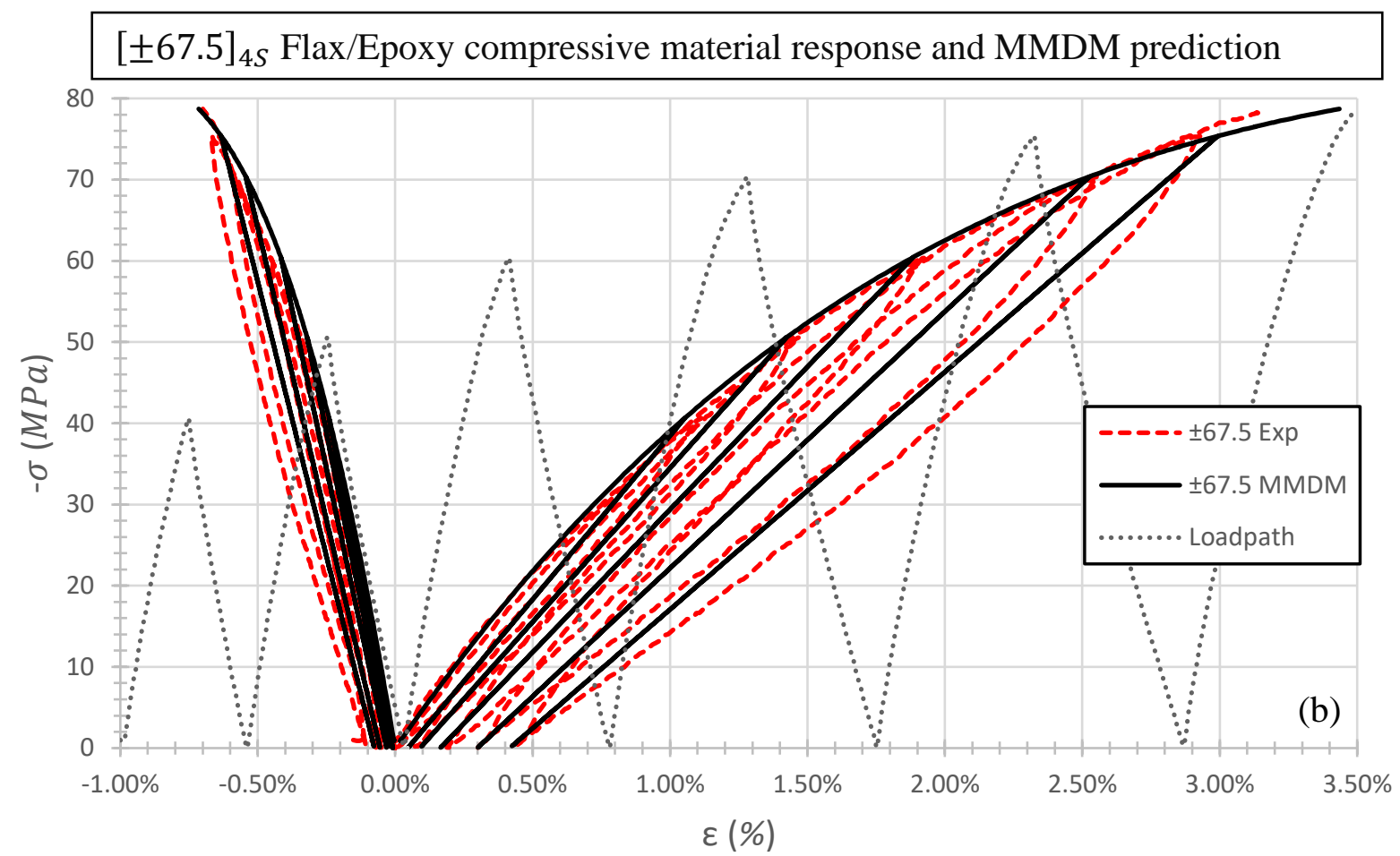

Figure 5-11: $\pm 67.5^{0}$ fiber-oriented Flax/Epoxy experimental behaviour and MMDM prediction

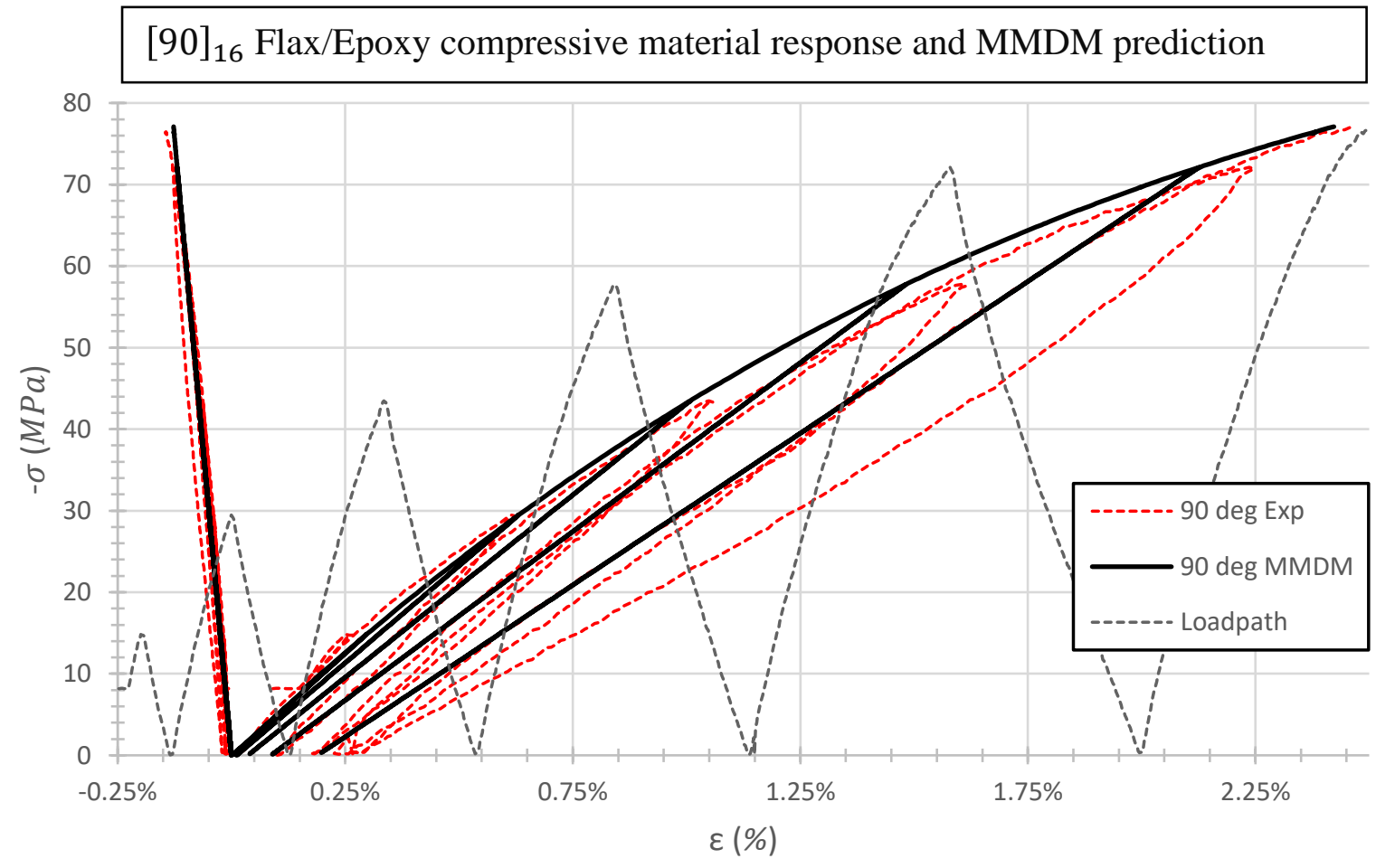

Figure 5-12: $90^{\circ}$ fiber-oriented Flax/Epoxy experimental behaviour and MMDM prediction 


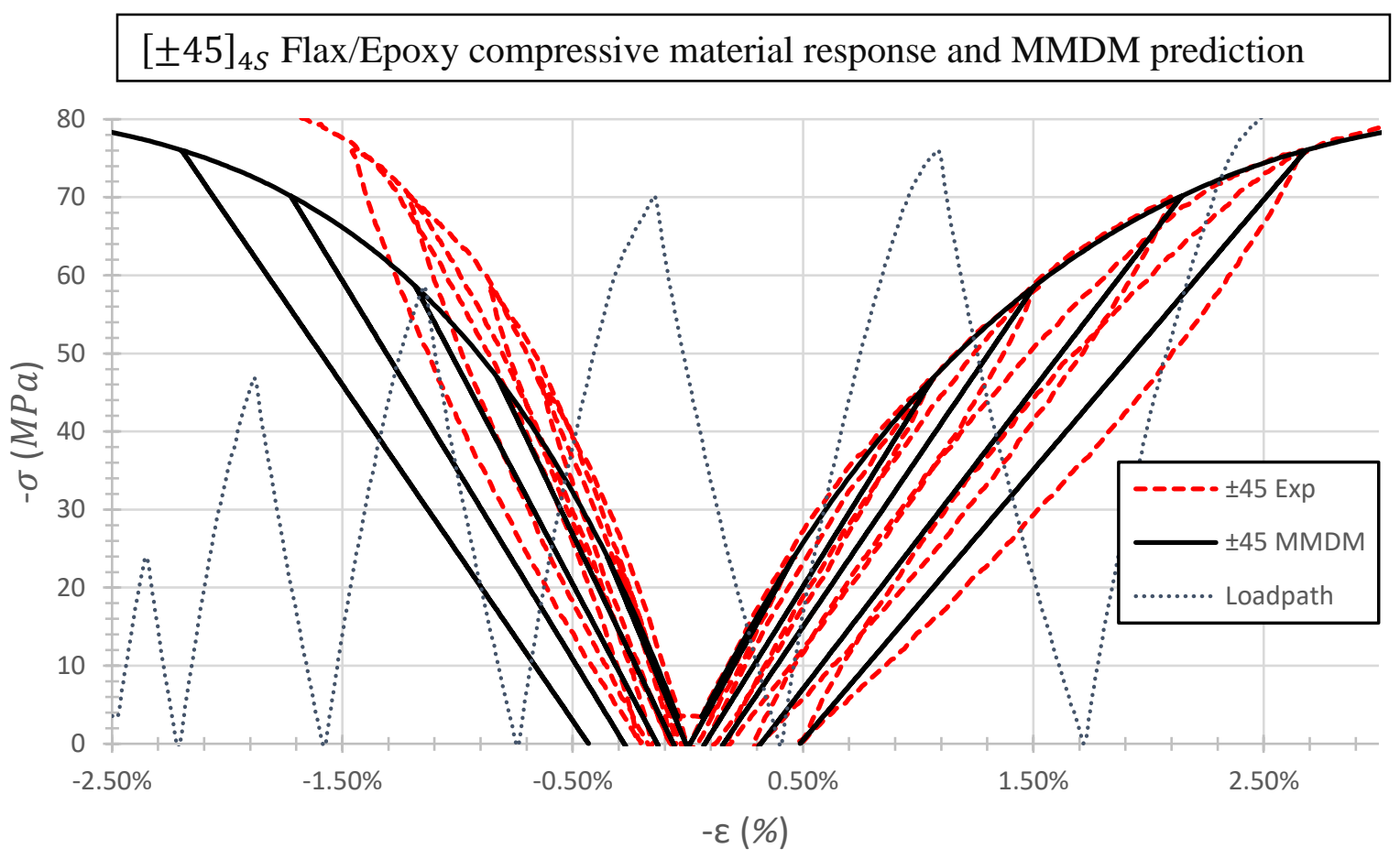

Figure 5-13: \pm 45 fiber-oriented Flax/Epoxy experimental behaviour and MMDM prediction

\subsection{Limitations}

It is important to note that there are a few limitations associated with the current model; which need to be taken into account for real design applications. As previously mentioned, the samples with $90^{\circ}$ - oriented fibers experience a plasticity evolution following a trend that is different from other fiber orientations. Due to the fact that shear and transverse plasticity evolutions are coupled within a single evolution law with a constant coupling parameter, means that a compromise must be established where either the transverse or the shear will lose accuracy. In this case, under the current formulation, the transverse strain response to compression can't be accurately decomposed into its elastic and plastic components. Nonetheless, the model does accurately predict the overall strain state; therefore, if the total strain rate is the only information required for a design task at hand, then the model can provide this information for transverse fiberoriented samples.

The model can accurately predict the longitudinal strain of the $\pm 45^{0}$ fiber-oriented samples, and cannot do it for the transverse direction. There are several possible explanations, and the true 
reason could be a combination of the following reasons: 1) damage mechanisms that are not accounted for in the constitutive equations such as inter-laminar re-orientation, severe delamination, and buckling. 2) DIC techniques are known to be less reliable in the direction perpendicular to the load axis, especially considering that the composite's poison's ratio varies severely at low stress rates before approaching a steady value at higher loads. A comprehensive investigation would be required in future works to determine the exact cause of this discrepancy

Finally, the reader should be reminded that even thought he model is built for a 3D volumetric element, it has been applied in this thesis only in 2D. Therefore, one should keep in mind that the model presented here is meant for in-plane load conditions. 


\section{Chapter 6: Conclusion and Future Work}

\subsection{Conclusion}

Natural fibers such as flax, present an untapped source of environmentally friendly, sustainable, and cost-effective substitutes to synthetic fibers used for composite reinforcements. Moreover, flax fibers have been shown to have comparable mechanical properties to E-Glass, the most popular choice of fiber reinforcement. The impeding factor of NFC's widespread use as loadbearing components is due to a noticeable lack data on their mechanical behaviour in compression, and a lack of robust and accurate predictive tools.

In this paper, quasi-static compressive testing was performed on Flax/Epoxy laminates of four principle orientations: $[0]_{16},[90]_{16},[ \pm 45]_{4 S}$, and $[ \pm 67.5]_{4 S}$. Experimental data were used to characterize the material behaviour in compression. It was found that Flax/Epoxy laminates experience linear damage and power-law plasticity evolution behaviours. This information was further used to modify the standard Mesoscale Damage Theory, allowing it to capture the nonlinear mechanical behaviour of Flax/Epoxy composites in compression. The modified mesoscale damage theory was integrated into open-source SMART+ material libraries, creating a robust and accurate tool capable of predicting the in-plane compressive behaviour of flax composites. This predictive tool was further validated on E-glass and Flax composites in compression as well as on E-glass/Epoxy composites in tension. Overall, the Modified Mesoscale Damage Model was found to be suitable for predicting the compressive behaviour of both synthetic and natural fiber composites. Being able to accurately model the non-linear mechanical behaviour of NFCs, will allow engineers to design with confidence components and parts from natural fibers. This will further promote the use of sustainable and environmentally friendly materials for structural applications.

\subsection{Future Work}

The MMDM has shown to be a robust tool in predicting the overall in-plane material response to compressive loads. Test specimens have been observed to buckle during compressive testing, and this phenomenon was not taken into accounted in the formulation of constitutive equations in the modified mesoscale damage model. In order to improve the model and fully capture the material behaviour up to failure, bucking effects as well as other out-of-plane effects 
must be accounted for. The model was formulated in a way that allows the effects of temperature to be taken into account, however, in this study, it has only been validated for room temperature. Therefore, future works may include formulating buckling constitutive equations, and performing mechanical tests at various service temperatures, and validating the model for thermal and buckling effects.

The second recommendation for future works is to implement the model into a finite element design software such as ANSYS or ABAQUS, and expand its application from a single element to complex finite element structures. The successful completion of the above suggestions, will result in a sophisticated model ready for mainstream structural applications. 


\section{Appendix A: Sample Calculations}

This appendix presents sample calculations performed in order to derive the optimizer's initial guess for material-specific compressive parameters. The superscript ' $c$ ' was dropped in order to reduce the congestion of scripts in the following formulas.

For the sake of space and paper conservation, repetition will be avoided where applicable; i.e. all relevant calculations will be shown, while calculations that require the repeated use of the same formula will not be shown.

\section{Fiber Direction ( $0^{\mathbf{0}}$ )}

\section{Damage Evolution}

The initial undamaged elastic modulus was found from the initial segment of the stress/strain curve using Eq. 4.26:

$$
E_{11}^{0}(n)=\frac{\sigma_{11}^{\prime \prime}-\sigma_{11}^{\prime}}{\varepsilon_{11}^{\prime \prime}-\varepsilon_{11}^{\prime \prime}}
$$

Monotonic 1 ; i.e. $(m 1)$ :

$$
E_{11}^{0}(m 1)=\frac{\sigma_{11}^{\prime \prime}-\sigma_{11}^{\prime}}{\varepsilon_{11}^{\prime \prime}-\varepsilon_{11}^{\prime \prime}}=\frac{-13.377+0.392}{(-42.77+6.111) * 10^{-5}} \frac{M P a}{\frac{m m}{m m}}=35,415 \mathrm{MPa}
$$

The actual undamaged elastic modulus was taken as an average of the five tests, as seen in Eq. 4.27:

$$
\begin{gathered}
E_{11}^{0}=\sum_{n=1}^{n=5} \frac{E_{11}^{0}(n)}{5}=\frac{35415.92+26038.42+30728.45+30410.12+29001.78}{5} \\
=30,318.94 \mathrm{MPa}
\end{gathered}
$$

The undamaged Poisson's ratio was found using Eq. 4.28 and 4.29, in accordance with the following steps: 1) the Poisson's ratio was found for every load increment of each test, 2) the median value of each test was found, 3) the undamaged Poisson's ratio was taken as the average of the five means.

Monotonic 1:

$$
\text { mean } v_{12}^{0}(m 1)=\text { mean }\left[-\left(\frac{\varepsilon_{y}}{\varepsilon_{x}}\right)_{i}\right], \text { where } i \leq 0, \quad i \in Z
$$


The actual Poisson's ratio was taken as an average of the five tests:

$$
v_{12}^{0}=\sum_{n=1}^{n=5} \frac{v_{12}^{0}(n)}{5}=\frac{0.446+0.353+0.455+0.380+0.346}{5}=0.396
$$

The ultimate stresses and strains were calculated as the average of the largest absolute stress/strain recorded for each specimen using Eq. 4.30:

$$
\begin{gathered}
\sigma_{11}^{u}=\frac{\sum_{n=1}^{n=5} \min \left(\sigma_{11}(n)\right)}{5}=\frac{-122.36-133.16-119.99-131.12-128.95}{5} \\
=-127.11 M P a \\
\varepsilon_{L L}^{u}=\varepsilon_{11}^{u}=\frac{\sum_{n=1}^{n=5} \min \left(\varepsilon_{11}(n)\right)}{5}=\frac{-1.51-1.55-1.88-1.95-1.13}{5}=-1.6 \% \\
\varepsilon_{T T}^{u}=\frac{\sum_{n=1}^{n=5} \max \left(\varepsilon_{T T}(n)\right)}{5}=\frac{0.71+0.62+0.7+0.83+0.4}{5}=0.65 \%
\end{gathered}
$$

The following calculations relate to the load/unload experiments performed on specimens with $0^{0}$ orientation, more specifically, on test 3 . Similar calculations were performed on tests 4 and 5 :

Points of interest were isolated for the upper and lower limits of the cycle using Eq. 4.31:

\begin{tabular}{|c|c|c|c|c|c|c|c|c|c|}
\hline \multirow[t]{2}{*}{ Point } & \multicolumn{3}{|c|}{ Upper limit of cycle } & \multicolumn{3}{|c|}{ Lower limit of cycle } & \multicolumn{3}{|c|}{ Elastic Moduli } \\
\hline & $\begin{array}{l}\sigma_{x} \\
(M P a)\end{array}$ & $\begin{array}{l}\varepsilon_{x} * 10^{3} \\
\left(\frac{m m}{m m}\right)\end{array}$ & $\begin{array}{l}\varepsilon_{y} * \\
10^{3} \\
\left(\frac{m m}{m m}\right)\end{array}$ & $\begin{array}{l}\sigma_{x} \\
(M P a)\end{array}$ & $\begin{array}{l}\varepsilon_{x} * \\
10^{3} \\
\left(\frac{m m}{m m}\right)\end{array}$ & $\begin{array}{l}\varepsilon_{y} * \\
10^{3} \\
\left(\frac{m m}{m m}\right)\end{array}$ & Rise $_{11}(M P a)$ & $\begin{array}{l}\text { Run }_{11} \\
* 10^{3}\left(\frac{\mathrm{mm}}{\mathrm{mm}}\right)\end{array}$ & $\begin{array}{l}\text { Run }_{22} \\
* 10^{3}\left(\frac{m m}{m m}\right)\end{array}$ \\
\hline 1 & 0.000 & 0.000 & 0.000 & -3.249 & 0.000 & 0.086 & 0.000 & 0.000 & 0.000 \\
\hline 2 & -25.646 & -0.923 & 0.407 & 0.000 & -0.161 & 0.091 & -25.646 & -0.762 & 0.316 \\
\hline 3 & -46.208 & -1.722 & 0.753 & -0.048 & -0.240 & 0.121 & -46.160 & -1.482 & 0.632 \\
\hline 4 & -66.770 & -2.557 & 1.190 & -0.832 & -0.375 & 0.171 & -65.938 & -2.182 & 1.019 \\
\hline 5 & -77.078 & -3.248 & 1.475 & -0.832 & -0.519 & 0.209 & -76.245 & -2.729 & 1.266 \\
\hline 6 & -87.604 & -4.057 & 1.847 & -1.174 & -0.597 & 0.276 & -86.430 & -3.460 & 1.571 \\
\hline 7 & -119.988 & -17.546 & 6.719 & & & & & & \\
\hline
\end{tabular}

$$
\begin{aligned}
\text { Rise }_{i} & =\left(\sigma^{U}-\sigma^{L}\right)_{i} \\
\text { Run }_{i} & =\left(\varepsilon^{U}-\varepsilon^{L}\right)_{i}
\end{aligned}
$$

where superscripts $U, L, D, p$, and 0 stand for Upper, Lower, Damaged, plastic, and undamaged respectively.

Table A-0-1: $0^{0}$ Points of interest, moduli rise and run

The damaged elastic modulus is calculated using Eq. 4.32, and further used to find the apparent plastic strain: 


$$
\begin{gathered}
E_{i}^{D}=\frac{\text { Rise }_{i}}{\text { Run }_{i}} \\
\varepsilon_{i}^{p}=\left(\varepsilon^{L}-\left(\frac{\sigma^{L}}{E^{D}}\right)\right)_{i}
\end{gathered}
$$

Damage in principal directions and thermodynamic damage energy release rates were calculated using Eqs. 4.34 and 4.35:

$$
\begin{gathered}
D_{i}=1-\frac{E_{i}^{D}}{E_{i}^{0}} \\
Y_{11}=\frac{\sigma_{11}^{{ }^{2}}}{2 E_{11}^{0}\left(1-D_{11}\right)^{2}}
\end{gathered}
$$

Table A-0-2: $0^{0}$ Elastic moduli, plastic strain, damage, and thermodynamic force

\begin{tabular}{|l|l|l|c|c|c|c|c|}
\hline \multicolumn{2}{|l|}{ Elastic moduli } & \multicolumn{2}{l|}{ Plastic strain (apparent) } & \multicolumn{2}{l|}{ Damage } \\
\hline $\boldsymbol{E}_{\mathbf{1 1}}^{\boldsymbol{D}}(\boldsymbol{M P a})$ & $E_{22}^{D}(\mathrm{MPa})$ & $\begin{array}{l}\varepsilon_{11}^{p} \\
* 10^{4}\left(\frac{m m}{m m}\right)\end{array}$ & $\begin{array}{l}\varepsilon_{22}^{p} \\
* 10^{4}\left(\frac{m m}{m m}\right)\end{array}$ & $D_{11}$ & $D_{22}$ & $Y_{11}(\sigma)(M P a)$ & $\sqrt{Y_{11}(\sigma)}(\sqrt{M P a})$ \\
\hline 34338.000 & 98617.000 & 0.000 & 0.000 & 0.000 & 0.000 & 0 & 0 \\
\hline 33671.205 & 81078.123 & -1.613 & 0.908 & 0.019 & 0.178 & 0.00996 & 0.099802 \\
\hline 31144.108 & 73064.935 & -2.383 & 1.219 & 0.093 & 0.259 & 0.037795 & 0.194408 \\
\hline 30214.242 & 64710.186 & -3.474 & 1.835 & 0.120 & 0.344 & 0.083846 & 0.289562 \\
\hline 27940.318 & 60227.295 & -4.894 & 2.226 & 0.186 & 0.389 & 0.130659 & 0.361467 \\
\hline 24982.023 & 55015.370 & -5.504 & 2.972 & 0.272 & 0.442 & 0.211123 & 0.459482 \\
\hline
\end{tabular}

The root of the thermodynamic force was plotted versus the accumulated damage $\left(\sqrt{Y_{11}(\sigma)}\right.$ vs $D_{11}$ ). The cumulative resulting plot was used to determine the initial guess values for $Y_{f}^{0}$ and $Y_{f}^{C}$.

\section{Plasticity Evolution}

Shear integrity for each point of interest was calculated, and used to find incremental and accumulated effective equivalent plastic strains using Eqs. 4.36-4.38:

$$
\begin{gathered}
\Omega_{i}=\left(1-D_{L}\right)_{i} \\
(\Delta p)_{i}=\Delta \varepsilon_{L}^{p}=-\frac{1}{2} \frac{\left(\varepsilon_{L_{i+1}}^{p}-\varepsilon_{L_{i}}^{p}\right)}{\Omega_{i+1}+\Omega_{i}} \\
p_{i}=p_{i-1}+(\Delta p)_{i}
\end{gathered}
$$

The equivalent stress yield function points for plotting, were calculated using Eq. 4.39: 


$$
f_{i}=\left(R_{i}+R_{0}\right)=-\frac{\sigma_{L_{i}}}{\Omega_{i}}
$$

Once the yield function vs accumulated plastic strain was plotted, a power-shaped function (Eq. 4.40) was fitted to the data accumulated from all relevant sapless.

$$
R_{i}=\beta\left(p_{i}\right)^{\alpha}
$$

The error in between the experimental and predicted yield function points was calculated as follows:

$$
\text { error }=\frac{(\text { prediction }- \text { experimental })^{2}}{(\text { experimental })^{2}}
$$

Again, Excel's Solver function was used to minimize the error and between the predicted and experimental results, yielding the initial guess values for $\beta_{f}$ and $\alpha_{f}$.

Table A-0-3: $0^{0}$ Shear integrity, plastic strain, and yield function points

\begin{tabular}{|c|c|c|c|c|c|}
\hline $\begin{array}{l}\text { Shear } \\
\text { integrity }\end{array}$ & $\begin{array}{l}\text { Plastic Strain } \\
\text { Increment }\end{array}$ & $\begin{array}{l}\text { Accumulated Plastic } \\
\text { Strain }\end{array}$ & $\begin{array}{l}\text { Experimental points for } \\
\text { yield function }\end{array}$ & $\begin{array}{l}\text { Power } \\
\text { prediction for R }\end{array}$ & $\begin{array}{l}\text { Error } \\
\boldsymbol{\Omega}_{\boldsymbol{i}}\end{array}$ \\
\hline $\boldsymbol{\Delta}_{\boldsymbol{i}} * \mathbf{1 0}^{\mathbf{4}}$ & $\boldsymbol{p}_{\boldsymbol{i}} * \mathbf{1 0}^{\mathbf{4}}$ & $\boldsymbol{f}_{\boldsymbol{i}}(\boldsymbol{M P a})$ & $\boldsymbol{R}_{\boldsymbol{i}}(\boldsymbol{M} \boldsymbol{P a})$ & 0.000 \\
\hline 1.000 & 0.000 & 0.000 & 0.000 & 50.158 & 0.8424 \\
\hline 0.981 & 15.975 & 15.975 & 26.154 & 58.254 & 0.0206 \\
\hline 0.907 & 7.270 & 23.245 & 50.947 & 66.985 & 0.0137 \\
\hline 0.880 & 9.746 & 32.991 & 75.883 & 75.822 & 0.0398 \\
\hline 0.814 & 12.019 & 45.010 & 94.726 & 78.889 & 0.1189 \\
\hline 0.728 & 4.705 & 49.715 & 120.412 & & \\
\hline
\end{tabular}

\section{Transverse Fiber direction $\left(90^{\circ}\right)$}

The transverse-fiber tests were performed in the same way as the longitudinal ones; with the same number of monotonic and cyclic loadings. Therefore, the transverse undamaged elastic modulus, Poisson's ratio, ultimate stresses, and ultimate strains were calculated with the same procedure as in the fiber direction:

Monotonic 1:

$$
E_{22}^{0}(m 1)=\frac{\sigma_{22}^{\prime \prime}-\sigma_{22}^{\prime}}{\varepsilon_{22}^{\prime \prime}-\varepsilon_{22}^{\prime \prime}}=\frac{-6.604-0}{(-1.1792-0) * 10^{-3}} \frac{M P a}{\frac{m m}{m m}}=5600.44 \mathrm{MPa}
$$

Average transverse undamaged elastic modulus is equal to:

$$
E_{22}^{0}=\sum_{n=1}^{n=5} \frac{E_{22}^{0}(n)}{5}=\frac{5600.44+5665.54+5237.10+4980.02+7035.93}{5}=5703.81 \mathrm{MPa}
$$


Average transverse Poisson's ratio:

$$
v_{12}^{0}=\sum_{n=1}^{n=5} \frac{v_{12}^{0}(n)}{5}=\frac{0.058+0.077+0.057+0.079+0.057}{5}=0.066
$$

Transverse ultimate stresses and strains were calculated using the same procedure as seen in for the fiber direction:

$$
\begin{gathered}
\sigma_{22}^{u}=\frac{\sum_{n=1}^{n=5} \min \left(\sigma_{22}(n)\right)}{5}=\frac{-75.23-72.00-77.1-75.81-99.5}{5}=-79.94 \mathrm{MPa} \\
\varepsilon_{L L}^{u}=\varepsilon_{22}^{u}=\frac{\sum_{n=1}^{n=5} \min \left(\varepsilon_{22}(n)\right)}{5}=\frac{-2.36-2.47-2.46-2.12-3.63}{5}=-2.61 \% \\
\varepsilon_{T T}^{u}=\frac{\sum_{n=1}^{n=5} \min \left(\varepsilon_{T T}(n)\right)}{5}=\frac{0.13+0.19+0.15+0.15+0.20}{5}=0.16 \%
\end{gathered}
$$

\section{$\pm 45^{0}$ Orientation}

Three $\pm 45^{0}$ orientation specimens where tested. Parameter $B$ was first calculated using Eq. 4.42, noting that the tension shear modulus of $\pm 45^{0}$ flax composites presented by Mahboob [43] was used in this calculation:

$$
\begin{gathered}
m=\cos \theta=\cos 45=0.707 \\
n=\sin \theta=0.5 \\
B=\left[\frac{\left(m^{2}\left(2 m^{2}-1\right)+\frac{4 m^{2} n^{2} G_{12}^{T}}{E_{22}}\left(\frac{E_{22}}{E_{11}} v_{12}+1\right)\right)}{\frac{4 m^{2} n^{2} G_{12}^{T}}{E_{22}}\left(\frac{E_{22}}{E_{11}}+2 \frac{E_{22}}{E_{11}} v_{12}+1\right)+\left(2 m^{2}-1\right)\left(m^{2}-n^{2}\right)}\right] \\
B=\left[\begin{array}{c}
\left(0.707^{2}\left(2(0.707)^{2}-1\right)+\frac{4(0.707)^{2}(0.707)^{2} 2.07}{5.7}\left(\frac{5.7}{30.32} 0.353+1\right)\right) \\
\frac{4(0.707)^{2}(0.707)^{2} 2.07}{5.7}\left(\frac{5.7}{30.32}+2 \frac{5.7}{30.32} 0.353+1\right)+\left(2(0.707)^{2}-1\right)\left((0.707)^{2}-(0.707)^{2}\right)
\end{array}\right]
\end{gathered}
$$

Parameter $k$ was found using Eq. 4.43 is equal to:

$$
k=-\frac{1}{2 m n}\left(B\left(1-2 m^{2}\right)+m^{2}=-0.5\right.
$$

Points of interest were isolated as previously done for the $0^{0}$ orientation. Stresses and strains in principal material coordinates for each increment were found as seen in Eqs. 4.44 and 4.45: 


$$
\begin{gathered}
\sigma_{11_{i}}=B \bar{\sigma}_{x_{i}} \quad \sigma_{22_{i}}=B \bar{\sigma}_{y_{i}} \quad \tau_{12_{i}}=k \bar{\sigma}_{x_{i}} \\
\varepsilon_{11}=m^{2} \varepsilon_{x}+n^{2} \varepsilon_{y} \quad \varepsilon_{22}=n^{2} \varepsilon_{x}+m^{2} \varepsilon_{y} \quad \varepsilon_{12}=-n m\left(\varepsilon_{x}-\varepsilon_{y}\right) \quad \gamma_{12}=2 \varepsilon_{12}
\end{gathered}
$$

The following calculations are related to test 2 . Components, and similar calculations have been performed for test 3:

\begin{tabular}{|c|c|c|c|c|c|c|c|c|c|}
\hline $\begin{array}{l}\sigma_{x} \\
(\boldsymbol{M P a})\end{array}$ & $\begin{array}{l}\boldsymbol{\varepsilon}_{\boldsymbol{x}}^{*} \\
\mathbf{1 0}^{3} \\
\left(\frac{m m}{m m}\right)\end{array}$ & $\begin{array}{l}\varepsilon_{y} * 10^{3} \\
\left(\frac{m m}{m m}\right)\end{array}$ & $\begin{array}{l}\sigma_{11} \\
(M P a)\end{array}$ & $\begin{array}{l}\varepsilon_{11} x 10^{3} \\
\left(\frac{m m}{m m}\right)\end{array}$ & $\begin{array}{l}\sigma_{22} \\
(M P a)\end{array}$ & $\begin{array}{l}\varepsilon_{22} * \\
10^{3} \\
\left(\frac{m m}{m m}\right)\end{array}$ & $\begin{array}{l}\tau_{12} \\
(M P a)\end{array}$ & $\begin{array}{l}\varepsilon_{12} * \\
\mathbf{1 0}^{3} \\
\left(\frac{m m}{m m}\right)\end{array}$ & $\begin{array}{l}\gamma_{12} * 10^{3} \\
(\mathrm{MPa})\end{array}$ \\
\hline 0.000 & 0.000 & 0.000 & 0.000 & 0.000 & 0.000 & 0.000 & 0.000 & 0.000 & 0.000 \\
\hline-23.961 & -4.927 & 2.981 & -19.346 & -0.973 & -4.615 & -0.973 & -11.981 & -3.954 & -7.908 \\
\hline-47.088 & -11.074 & 6.138 & -38.019 & -2.468 & -9.069 & -2.468 & -23.544 & -8.606 & -17.212 \\
\hline-58.813 & -15.556 & 8.100 & -47.486 & -3.728 & -11.327 & -3.728 & -29.407 & -11.828 & -23.656 \\
\hline-70.291 & -21.522 & 10.940 & -56.754 & -5.291 & -13.537 & -5.291 & -35.146 & -16.231 & -32.461 \\
\hline-76.342 & -26.249 & 13.027 & -61.639 & -6.611 & -14.703 & -6.611 & -38.171 & -19.638 & -39.276 \\
\hline-87.709 & -51.121 & 25.690 & -70.817 & -12.715 & -16.892 & -12.715 & -43.854 & -38.405 & -76.811 \\
\hline
\end{tabular}

Table A-0-4: $\pm 45^{0}$ Upper limit points of interest

\begin{tabular}{|c|c|c|c|c|c|c|c|c|c|}
\hline $\begin{array}{l}\sigma_{x} * 10^{3} \\
(M P a)\end{array}$ & $\begin{array}{l}\varepsilon_{x} \times 10^{3} \\
\left(\frac{m m}{m m}\right)\end{array}$ & $\begin{array}{l}\varepsilon_{y} x 10^{3} \\
\left(\frac{m m}{m m}\right)\end{array}$ & $\begin{array}{l}\sigma_{11} * \\
10^{3} \\
(M P a)\end{array}$ & $\begin{array}{l}\varepsilon_{11} \times 10^{3} \\
\left(\frac{m m}{m m}\right)\end{array}$ & $\begin{array}{l}\sigma_{22} * 10^{3} \\
(\mathrm{MPa})\end{array}$ & $\begin{array}{l}\varepsilon_{22} x 10^{3} \\
\left(\frac{m m}{m m}\right)\end{array}$ & $\begin{array}{l}\tau_{12} * 10^{3} \\
(\mathrm{MPa})\end{array}$ & $\begin{array}{l}\varepsilon_{12} \times 10^{3} \\
\left(\frac{m m}{m m}\right)\end{array}$ & $\begin{array}{l}\gamma_{12} * 10^{3} \\
(\mathrm{MPa})\end{array}$ \\
\hline 0.000 & 0.000 & 0.000 & 0.000 & 0.000 & 0.000 & 0.000 & 0.000 & 0.000 & 0.000 \\
\hline 5.874 & 0.000 & 0.000 & 0.005 & 0.000 & 1.131 & 0.000 & 2.937 & 2.937 & 0.000 \\
\hline 364.204 & -0.384 & -0.384 & 0.294 & -0.020 & 70.142 & -0.020 & 182.102 & 182.102 & -0.364 \\
\hline 381.827 & -1.744 & -1.744 & 0.308 & -0.447 & 73.536 & -0.447 & 190.914 & 190.914 & -1.296 \\
\hline 140.982 & -2.722 & -2.722 & 0.114 & -0.524 & 27.152 & -0.524 & 70.491 & 70.491 & -2.198 \\
\hline 82.240 & -4.652 & -4.652 & 0.066 & -1.205 & 15.839 & -1.205 & 41.120 & 41.120 & -3.447 \\
\hline
\end{tabular}

Table A-0-5: $\pm 45^{0}$ Lower limit points of interest

Rises and runs for this fiber orientation were calculated as previously seen in the $0^{0}$ calculations, and tabulated in Table A-0-6:

\begin{tabular}{|c|c|c|c|c|c|c|}
\hline \multicolumn{2}{|c|}{ Fibre direction (11) } & \multicolumn{2}{|c|}{ Transverse direction (22) } & \multicolumn{3}{|c|}{ Shear direction (12) } \\
\hline $\begin{array}{l}\text { Rise } \\
\boldsymbol{\sigma}_{\mathbf{1 1}}(\boldsymbol{M P a})\end{array}$ & $\begin{array}{l}\text { Run } \varepsilon_{11} * \\
10^{4}(\mathrm{~mm} / \\
\mathrm{mm})\end{array}$ & $\begin{array}{l}\text { Rise } \\
\sigma_{22}(M P a)\end{array}$ & $\begin{array}{l}\text { Run } \varepsilon_{11} * \\
10^{2}(\mathrm{~mm} / \\
\mathrm{mm})\end{array}$ & Rise $\tau_{12}(M P a)$ & $\begin{array}{l}\text { Run } \varepsilon_{12} * \\
10^{2}(\mathrm{~mm} / \\
\mathrm{mm})\end{array}$ & $\begin{array}{l}\text { Run } \gamma_{12} * \\
10^{2}(\mathrm{~mm} / \\
\mathrm{mm})\end{array}$ \\
\hline 0.000 & 0.000 & 0.000 & 0.000 & 0.000 & 0.000 & 0.000 \\
\hline-5.037 & 2.011 & -33.445 & -0.739 & 13.007 & -0.380 & -0.760 \\
\hline-7.142 & 2.202 & -47.425 & -1.170 & 18.444 & -0.596 & -1.192 \\
\hline-8.517 & 0.204 & -56.554 & -1.579 & 21.994 & -0.791 & -1.581 \\
\hline-9.900 & -1.383 & -65.735 & -2.125 & 25.564 & -1.056 & -2.112 \\
\hline-10.631 & -1.436 & -70.586 & -2.327 & 27.451 & -1.157 & -2.313 \\
\hline
\end{tabular}

Table A-0-6 $\pm 45^{\circ}$ : Rises and runs for points of interest

The damaged elastic moduli and apparent plastic strains were calculated as preciously seen in the $0^{0}$ section, and tabulated in Table A-0-7: 
Table A-0-7: $\pm 45^{0}$ Damaged elastic moduli and plastic strains

\begin{tabular}{|c|c|c|c|c|c|c|c|}
\hline \multicolumn{4}{|c|}{ Damaged elastic moduli } & \multicolumn{4}{|c|}{ Plastic strain } \\
\hline$E_{11}^{D}(M P a)$ & $E_{22}^{D}(M P a)$ & $G_{12}^{D}(\varepsilon)(M P a)$ & $G_{12}^{D}(\gamma)(M P a)$ & $\begin{array}{l}\varepsilon_{11}^{p} \\
* 10^{5}\left(\frac{m m}{m m}\right)\end{array}$ & $\begin{array}{l}\varepsilon_{22}^{p} \\
* 10^{5}\left(\frac{m m}{m m}\right)\end{array}$ & $\begin{array}{l}\varepsilon_{12}^{p} \\
* 10^{5}\left(\frac{\mathrm{mm}}{\mathrm{mm}}\right)\end{array}$ & $\begin{array}{l}\gamma_{11}^{p} \\
* 10^{5}\left(\frac{\mathrm{mm}}{\mathrm{mm}}\right)\end{array}$ \\
\hline 75610.000 & 13486.000 & 4045.400 & 2022.700 & 0.000 & 0.000 & 0.000 & 0.000 \\
\hline 19893.762 & 4745.212 & 3030.682 & 1515.341 & -0.024 & -0.024 & -0.097 & -0.194 \\
\hline 15653.334 & 3733.753 & 2878.761 & 1439.380 & -3.874 & -3.874 & -42.742 & -85.484 \\
\hline 14567.626 & 3474.782 & 2810.376 & 1405.188 & -46.835 & -46.835 & -136.428 & -272.855 \\
\hline 11929.216 & 2845.448 & 2509.663 & 1254.831 & -53.347 & -53.347 & -222.637 & -445.275 \\
\hline 11414.123 & 2722.584 & 2360.138 & 1180.069 & -121.069 & -121.069 & -346.468 & -692.935 \\
\hline
\end{tabular}

Damage in principal directions were calculated using the damaged and undamaged elastic moduli as seen in Eq. 4.46. Following which, the thermodynamic damage energy release rates were calculated using Eq. 4.47. The results can be seen in Table A-0-8:

$$
D_{i}=1-\frac{E_{i}^{D}}{E_{i}^{0}} \quad \text { and } \quad D_{12}=1-\frac{G_{12}^{D}}{G_{12}^{0}} \quad \text { and } \quad Y_{12}=\frac{\tau_{11}^{{ }^{2}}}{2 G_{12}^{0}(\gamma)\left(1-D_{12}(\gamma)\right)^{2}}
$$

where $G_{12}^{0}(\gamma)$ and $D_{12}(\gamma)$ are shear modulus and damage calculated form $\gamma$ rather than $\varepsilon$.

Table A-0-8: $\pm 45^{\circ}$ Calculated damages and thermodynamic force conjugates

\begin{tabular}{|c|c|c|c|c|c|}
\hline Damage & \multicolumn{3}{|c|}{ Thermodynamic force conjugate of damage } \\
\hline $\boldsymbol{D}_{\mathbf{1 1}}$ & $\boldsymbol{D}_{\mathbf{2 2}}$ & $\boldsymbol{D}_{\mathbf{1 2}}\left(\boldsymbol{\varepsilon}_{\mathbf{1 2}}\right)$ & $\boldsymbol{D}_{\mathbf{1 2}}\left(\boldsymbol{\gamma}_{\mathbf{1 2}}\right)$ & $\boldsymbol{Y}_{\mathbf{1 2}}(\boldsymbol{M P a})$ & $\sqrt{\boldsymbol{Y}_{\mathbf{1 2}}}(\sqrt{\mathbf{M P a}})$ \\
\hline 0 & 0 & 0 & 0 & 0 & 0 \\
\hline 0.7368898 & 0.648138 & 0.250833 & 0.250833 & 0.063217 & 0.25143 \\
\hline 0.7929727 & 0.723139 & 0.288387 & 0.288387 & 0.270591 & 0.520183 \\
\hline 0.807332 & 0.742342 & 0.305291 & 0.305291 & 0.442916 & 0.665519 \\
\hline 0.842227 & 0.789007 & 0.379626 & 0.379626 & 0.79337 & 0.890713 \\
\hline 0.8490395 & 0.798118 & 0.416587 & 0.416587 & 1.058164 & 1.028671 \\
\hline
\end{tabular}

The thermodynamic coupling parameter $A_{t s}$ was found using Eqs. 4.48 and 4.49:

$$
\left(A_{t s}\right)_{i}=\frac{\left(\varepsilon_{22_{i}}^{p}-\varepsilon_{22_{i-1}}^{p}\right) \tau_{12_{i}}\left(1-D_{22_{i}}\right)^{2}}{\left(\gamma_{12_{i}}^{p}-\gamma_{12_{i-1}}^{p}\right) \sigma_{22_{i}}\left(1-D_{12_{i}}\right)^{2}}
$$

where $i$ denotes the number of the point of interest.

The average value for $A_{t s}$ for this test was taken:

$$
A_{t s}=\frac{\sum_{i=1}^{i=5} a^{2} i}{5}=0.053
$$

Shear integrity, effective equivalent plastic strain, and stress yield function points were calculated as previously shown in the $0^{0}$ calculations. An exponential function was fitted to the experimental 
data, the difference between the experimental and predicted yield function points was minimized, which yielded the initial guesses for $\beta$ and $\alpha$ of this fiber orientation.

Table A-0-9: $\pm 45^{0}$ Shear integrity, plastic strain, and yield function points

\begin{tabular}{|c|c|c|c|c|c|}
\hline $\begin{array}{c}\text { Shear } \\
\text { integrity }\end{array}$ & $\begin{array}{c}\text { Plastic Strain } \\
\text { Increment }\end{array}$ & $\begin{array}{c}\text { Accumulated } \\
\text { Plastic Strain }\end{array}$ & $\begin{array}{c}\text { Experimental points } \\
\text { for yield function }\end{array}$ & $\begin{array}{c}\text { Power law } \\
\text { prediction for R }\end{array}$ & Error \\
\hline $\boldsymbol{\Omega}_{\boldsymbol{i}}$ & $\Delta p_{i} * 10^{4}$ & $p_{i} * 10^{4}$ & $f_{i}(\mathrm{MPa})$ & $R_{i}(\mathrm{MPa})$ & 0 \\
\hline 1.000 & 0.000 & 0.000 & 0.000 & 0.000 & 0.0006 \\
\hline 0.749 & 0.017 & 0.017 & 15.992 & 39.585 & 0.0377 \\
\hline 0.712 & 6.230 & 6.246 & 33.085 & 47.228 & 0.0134 \\
\hline 0.695 & 13.175 & 19.422 & 42.329 & 50.773 & 0.0108 \\
\hline 0.620 & 11.337 & 30.759 & 56.652 & 54.031 & 0.0303 \\
\hline 0.583 & 14.907 & 45.666 & 65.427 & & 0.5 \\
\hline
\end{tabular}

The following calculations are related to test 2 - laminate level, and similar calculations have been performed for test 3 . The procedure is nearly identical to that seen in $0^{0}$ calculations, with the exception that there are calculations performed on both fiber and transverse direction. Therefore, calculations will be shown in detail, to avoid reader confusion.

Points of interest where singled out from stress-strain data, for upper and lower loop sections, and their rise and runs were calculated using Eq. 4.50:

$$
\text { Rise }_{L_{i}}=\left(\sigma_{x}{ }^{U}-\sigma_{x}{ }^{L}\right)_{i} \quad \operatorname{Run}_{L_{i}}=\left(\varepsilon_{x}{ }^{U}-\varepsilon_{x}{ }^{L}\right)_{i} \quad \operatorname{Run}_{T_{i}}=\left(\varepsilon_{y}{ }^{U}-\varepsilon_{y}{ }^{L}\right)_{i}
$$

Table A-0-10: $\pm 45^{0}$ Points of interested and their respective rise and run

\begin{tabular}{|c|c|c|c|c|c|c|c|c|}
\hline \multicolumn{3}{|c|}{ UPPER limit of cycle } & \multicolumn{3}{|c|}{ LOWER limit of cycle } & \multicolumn{3}{|c|}{ Elastic Moduli } \\
\hline \multirow{2}{*}{$\sigma_{L}^{U}(M P a)$} & \multirow{2}{*}{$\begin{array}{l}\varepsilon_{L}^{U} \\
* 10^{3} \\
\left(\frac{m m}{m m}\right)\end{array}$} & \multirow{2}{*}{$\begin{array}{c}\varepsilon_{T}^{U} * 10^{3} \\
\left(\frac{m m}{m m}\right)\end{array}$} & \multirow{2}{*}{$\begin{array}{l}\sigma_{L}^{L} \\
* 10^{3}(M P a)\end{array}$} & \multirow{2}{*}{$\begin{array}{c}\varepsilon_{L}^{L} * 10^{3} \\
\left(\frac{m m}{m m}\right)\end{array}$} & \multirow{2}{*}{$\begin{array}{l}\varepsilon_{T}^{L} \\
* 10^{3} \\
\left(\frac{m m}{m m}\right)\end{array}$} & \multicolumn{2}{|l|}{ Longitudinal } & \multirow{2}{*}{$\begin{array}{l}\text { Transverse } \\
\qquad \begin{array}{l}\text { Run } \\
\text { * } 10^{3}(\mathrm{~mm} \\
/ \mathrm{mm})\end{array}\end{array}$} \\
\hline & & & & & & Rise $_{L}(\mathrm{MPa})$ & $\begin{array}{l}\text { Run }_{L} \\
* 10^{3}(\mathrm{~mm} \\
/ \mathrm{mm})\end{array}$ & \\
\hline 0.000 & 0.000 & 0.000 & 0.000 & $\begin{array}{c}- \\
5592.299\end{array}$ & -1.476 & 0.000 & 0.000 & 0.000 \\
\hline-23.961 & -4.927 & 2.981 & 5.874 & 0.000 & 0.000 & -23.967 & -4.927 & 2.981 \\
\hline-47.088 & -11.074 & 6.138 & 364.204 & -0.384 & 0.344 & -47.452 & -10.689 & 5.794 \\
\hline-58.813 & -15.556 & 8.100 & 381.827 & -1.744 & 0.849 & -59.195 & -13.812 & 7.251 \\
\hline-70.291 & -21.522 & 10.940 & 140.982 & -2.722 & 1.674 & -70.432 & -18.799 & 9.265 \\
\hline-76.342 & -26.249 & 13.027 & 82.240 & -4.652 & 2.242 & -76.424 & -21.597 & 10.785 \\
\hline-87.709 & -51.121 & 25.690 & & & & & & \\
\hline
\end{tabular}

Next, the damaged elastic moduli, plastic strain, damage, and thermodynamic damage energy release rates were calculated as seen earlier, with corresponding subscripts $L \& T$ :

$$
E_{L}^{D}=\frac{\text { Rise }_{L}}{\text { Run }_{L}} \quad \& \quad E_{T}^{D}=\frac{\text { Rise }_{L}}{\text { Run }_{T}}
$$




$$
\begin{gathered}
\left(\varepsilon_{L}^{p}\right)_{i}=\left(\varepsilon_{L}^{L}-\left(\frac{\sigma_{L}^{L}}{E_{L}^{D}}\right)\right)_{i} \&\left(\varepsilon_{T}^{p}\right)_{i}=\left(\varepsilon_{T}^{L}-\left(\frac{\sigma_{L}^{L}}{E_{T}^{D}}\right)\right)_{i} \\
D_{L}=1-\frac{E_{L}^{D}}{E_{L}^{0}} \& \quad D_{T}=1-\frac{E_{T}^{D}}{E_{T}^{0}} \\
Y_{L}(\sigma)=\frac{\sigma_{L}^{2}}{2 E_{L}^{0}\left(1-D_{L}\right)^{2}}
\end{gathered}
$$

where subscripts $L$ and $T$ stand for longitudinal and transverse directions.

Table A-0-11: $\pm 45^{0}$ Damaged elastic moduli, plastic strain, damage, and energy release rates

\begin{tabular}{|c|c|c|c|l|c|c|c|}
\hline \multicolumn{2}{|c|}{ Damaged Elastic Moduli } & \multicolumn{3}{|c|}{ Plastic Strain (apparent) } & \multicolumn{1}{c|}{ Damage } & \multicolumn{1}{c|}{$\begin{array}{l}\text { Thermodynamic damage } \\
\text { energy release rate }\end{array}$} \\
\hline $\boldsymbol{E}_{\boldsymbol{L}}^{\boldsymbol{D}}(\boldsymbol{M P a})$ & $E_{T}^{D}(M P a)$ & $\varepsilon_{L}^{p}$ & $D_{L}$ & $D_{T}$ & $Y_{L}(\sigma)(M P a)$ & $\sqrt{Y_{L}(\sigma)}(\sqrt{M P a})$ \\
\hline $7410^{3}\left(\frac{m m}{m m}\right)$ & $\begin{array}{l}\varepsilon_{T}^{p} \\
* 10^{3}\left(\frac{m m}{m m}\right)\end{array}$ & & & & \\
\hline 4864.630 & 8039.020 & -0.001 & -0.001 & 0.345 & 0.098 & 0.208 & 0.456 \\
\hline 4439.185 & 8189.665 & -0.466 & 0.300 & 0.402 & 0.081 & 0.523 & 0.723 \\
\hline 4285.644 & 8164.119 & -1.833 & 0.802 & 0.423 & 0.084 & 0.944 & 0.971 \\
\hline 3746.534 & 7601.874 & -2.760 & 1.656 & 0.495 & 0.147 & 1.717 & 1.310 \\
\hline 3538.698 & 7086.460 & -4.675 & 2.231 & 0.523 & 0.205 & 2.049 & 1.431 \\
\hline
\end{tabular}

Shear integrity for each point of interest was calculated, and used to find incremental and accumulated effective equivalent plastic strains:

$$
\begin{gathered}
\Omega_{i}=\left(1-D_{L}\right)_{i} \\
(\Delta p)_{i}=\Delta \varepsilon_{L}^{p}=-\frac{1}{2} \frac{\left(\varepsilon_{L_{i+1}}^{p}-\varepsilon_{L_{i}}^{p}\right)}{\Omega_{i+1}+\Omega_{i}} \\
p_{i}=p_{i-1}+(\Delta p)_{i}
\end{gathered}
$$

The equivalent stress yield function points for plotting, were calculated as follows:

$$
f_{i}=R+R_{0}=-\frac{\sigma_{L_{i}}}{\Omega_{i}}
$$

Once the yield function vs accumulated plastic strain was plotted, a power law curve was fitted to the data.

$$
R_{i}=\beta *\left(p_{i}\right)^{\alpha}
$$

Following which, experimental and predicted points were compared, and their error was calculated: 


$$
\text { error }=\frac{\text { prediction }- \text { experimental }}{(\text { experimental })^{2}}
$$

In order to determine the parameters $\alpha \& \beta$, Microsoft Excel's 'Solver' was again used, which minimized the error by varying parameters $\alpha \& \beta$ up to convergence.

Table A-0-12: $\pm 45^{0}$ Shear integrity, plastic strain, and yield function points

\begin{tabular}{|c|c|c|c|c|c|}
\hline $\begin{array}{c}\text { Shear } \\
\text { integrity }\end{array}$ & $\begin{array}{c}\text { Plastic Strain } \\
\text { Increment }\end{array}$ & $\begin{array}{c}\text { Accumulated } \\
\text { Plastic Strain }\end{array}$ & $\begin{array}{c}\text { Experimental points } \\
\text { for yield function }\end{array}$ & $\begin{array}{c}\text { Power law } \\
\text { prediction for R }\end{array}$ & Error \\
\hline $\boldsymbol{\Omega}_{\boldsymbol{i}}$ & $\Delta p_{i} * 10^{4}$ & $p_{i} * 10^{4}$ & $f_{i}(\mathrm{MPa})$ & $R_{i}(\mathrm{MPa})$ & 0.000 \\
\hline 1.000 & 0.000 & 0.000 & 0.000 & 49.069 & 0.6796 \\
\hline 0.830 & 7.439 & 7.439 & 46.388 & 76.590 & 0.0365 \\
\hline 0.745 & 6.006 & 13.445 & 73.567 & 97.099 & 0.0044 \\
\hline 0.660 & 4.986 & 18.431 & 98.816 & 122.242 & 0.0084 \\
\hline 0.570 & 6.601 & 25.032 & 133.279 & 155.021 & 0.0023 \\
\hline 0.559 & 9.297 & 34.329 & 145.588 & & 0 \\
\hline
\end{tabular}

\section{$\pm 67.5^{0}$ Orientation}

There have been three cyclic-load type tests performed on samples of this orientation. The same approach was used as in the calculation of the $\pm 45^{\circ}$ case:

$$
\begin{gathered}
m=\cos \theta=\cos 67.5=0.382683 \\
n=\sin \theta=\sin 67.5=0.923879
\end{gathered}
$$

$B$

$$
\begin{aligned}
& =\left[\frac{\left(0.382^{2}\left(2(0.382)^{2}-1\right)+\frac{4(0.382)^{2}(0.924)^{2} 2.07}{5.7}\left(\frac{5.7}{30.32} 0.353+1\right)\right)}{\frac{4(0.382)^{2}(0.924)^{2} 2.07}{5.7}\left(\frac{5.7}{30.32}+2 \frac{5.7}{30.32} 0.353+1\right)+\left(2(0.382)^{2}-1\right)\left((0.382)^{2}-(0.924)^{2}\right)}\right] \\
& =0.13089
\end{aligned}
$$

$$
k=-\frac{1}{2 m n}\left(B\left(1-2 m^{2}\right)+m^{2}=-0.338\right.
$$

The following results are related to test 1 - components, and similar calculations have been performed for test 2 and 3:

Stresses and strains in the principal material coordinate system were calculated as previously seen in the \pm 45 section. 
Table A-0-13: $\pm 67.5^{0}$ Upper limit points of interest

\begin{tabular}{|c|c|c|c|c|c|c|c|c|c|}
\hline $\begin{array}{l}\sigma_{x} \\
(\boldsymbol{M P a})\end{array}$ & $\begin{array}{l}\varepsilon_{x} \\
\left(\frac{m m}{m m}\right)\end{array}$ & $\varepsilon_{y}\left(\frac{m m}{m m}\right)$ & $\begin{array}{l}\sigma_{11} \\
(M P a)\end{array}$ & $\begin{array}{l}\varepsilon_{11} x 10^{-4} \\
\left(\frac{m m}{m m}\right)\end{array}$ & $\begin{array}{l}\sigma_{22} \\
(M P a)\end{array}$ & $\begin{array}{l}\varepsilon_{22} \\
\left(\frac{m m}{m m}\right)\end{array}$ & $\begin{array}{l}\tau_{12} \\
(M P a)\end{array}$ & $\begin{array}{l}\varepsilon_{12} \\
\left(\frac{m m}{m m}\right)\end{array}$ & $\begin{array}{l}\gamma_{12} \\
(M P a)\end{array}$ \\
\hline 0.000 & 0.000 & 0.000 & 0.000 & 0.000 & 0.000 & 0.000 & 0.000 & 0.000 & 0.000 \\
\hline-38.482 & -0.010 & 0.002 & -5.037 & 2.100 & -33.445 & -0.008 & 13.007 & -0.004 & -0.008 \\
\hline-54.778 & -0.016 & 0.003 & -7.170 & 2.360 & -47.608 & -0.013 & 18.515 & -0.007 & -0.013 \\
\hline-65.180 & -0.021 & 0.004 & -8.531 & 0.380 & -56.648 & -0.018 & 22.031 & -0.009 & -0.018 \\
\hline-76.035 & -0.029 & 0.005 & -9.952 & 1.360 & -66.082 & -0.024 & 25.700 & -0.012 & -0.024 \\
\hline-81.443 & -0.033 & 0.005 & -10.660 & 2.260 & -70.783 & -0.027 & 27.528 & -0.014 & -0.027 \\
\hline-86.214 & -0.037 & 0.006 & -11.285 & 0.660 & -74.930 & -0.031 & 29.140 & -0.015 & -0.031 \\
\hline
\end{tabular}

Table A-0-14: $\pm 67.5^{0}$ Lower limit points of interest

\begin{tabular}{|c|c|c|c|c|c|c|c|c|c|}
\hline$\sigma_{x}(M P a)$ & $\begin{array}{l}\varepsilon_{x} x 1^{3} \\
\left(\frac{m m}{m m}\right)\end{array}$ & $\begin{array}{l}\varepsilon_{y} x 10^{4} \\
\left(\frac{m m}{m m}\right)\end{array}$ & $\begin{array}{l}\sigma_{11} \\
(M P a)\end{array}$ & $\begin{array}{l}\varepsilon_{11} x 10^{5} \\
\left(\frac{m m}{m m}\right)\end{array}$ & $\begin{array}{l}\sigma_{22} \\
(M P a)\end{array}$ & $\begin{array}{l}\varepsilon_{22} x 10^{3} \\
\left(\frac{m m}{m m}\right)\end{array}$ & $\begin{array}{l}\tau_{12} \\
(M P a)\end{array}$ & $\begin{array}{l}\varepsilon_{12} x 10^{4} \\
\left(\frac{m m}{m m}\right)\end{array}$ & $\begin{array}{l}\gamma_{12} \\
(M P a)\end{array}$ \\
\hline 0.000 & 0.000 & 0.000 & 0.000 & 0.000 & 0.000 & 0.000 & 0.000 & 0.000 & 0.000 \\
\hline 0.000 & -0.813 & 1.490 & 0.000 & 0.860 & 0.000 & -0.672 & 0.000 & -3.404 & -6.808 \\
\hline-0.211 & -1.630 & 2.980 & -0.028 & 1.556 & -0.183 & -1.348 & 0.071 & -6.820 & -13.640 \\
\hline-0.108 & -2.310 & 4.180 & -0.014 & 1.724 & -0.094 & -1.917 & 0.037 & -9.673 & -19.346 \\
\hline-0.400 & -3.490 & 6.030 & -0.052 & 0.257 & -0.348 & -2.895 & 0.135 & -14.488 & -28.976 \\
\hline-0.227 & -5.080 & 7.750 & -0.030 & -8.268 & -0.197 & -4.226 & 0.077 & -20.715 & -41.430 \\
\hline
\end{tabular}

Rises and runs for this fiber orientation were calculated as previously seen in the $0^{0}$ calculations, and tabulated in Table A-0-15:

Table A-0-15: $\pm 67.5^{0}$ Rises and runs for points of interest

\begin{tabular}{|c|c|c|c|c|c|c|}
\hline \multicolumn{2}{|c|}{ Fibre direction (11) } & \multicolumn{2}{|c|}{ Transverse direction (22) } & \multicolumn{3}{|c|}{ Shear direction (12) } \\
\hline $\begin{array}{l}\text { Rise } \\
\sigma_{11}(M P a)\end{array}$ & $\begin{array}{l}\text { Run } \varepsilon_{11} * \\
10^{4}\left(\frac{\mathrm{mm}}{\mathrm{mm}}\right)\end{array}$ & $\begin{array}{l}\text { Rise } \\
\sigma_{22}(M P a)\end{array}$ & $\begin{array}{l}\text { Run } \varepsilon_{11} * \\
10^{2}\left(\frac{\mathrm{mm}}{\mathrm{mm}}\right)\end{array}$ & Rise $\tau_{12}(M P a)$ & $\begin{array}{l}\text { Run } \varepsilon_{12} * \\
10^{2}\left(\frac{\mathrm{mm}}{\mathrm{mm}}\right)\end{array}$ & $\begin{array}{l}\text { Run } \\
10^{2}\left(\frac{\mathrm{mm}}{\mathrm{mm}}\right)\end{array} \gamma_{12} *$ \\
\hline 0.000 & 0.000 & 0.000 & 0.000 & 0.000 & 0.000 & 0.000 \\
\hline-5.037 & 2.011 & -33.445 & -0.739 & 13.007 & -0.380 & -0.760 \\
\hline-7.142 & 2.202 & -47.425 & -1.170 & 18.444 & -0.596 & -1.192 \\
\hline-8.517 & 0.204 & -56.554 & -1.579 & 21.994 & -0.791 & -1.581 \\
\hline-9.900 & -1.383 & -65.735 & -2.125 & 25.564 & -1.056 & -2.112 \\
\hline-10.631 & -1.436 & -70.586 & -2.327 & 27.451 & -1.157 & -2.313 \\
\hline
\end{tabular}

The damaged elastic moduli and apparent plastic strains were calculated as preciously seen in the $0^{0}$ section, and tabulated in Table A-0-16: 
Table A-0-16: $\pm 67.5^{0}$ Damaged elastic moduli and plastic strains

\begin{tabular}{|c|c|c|c|c|c|c|c|}
\hline \multicolumn{4}{|c|}{ Damaged elastic moduli } & \multicolumn{4}{|c|}{ Plastic strain } \\
\hline $\begin{array}{l}E_{11}^{D} \\
* 10^{-4}(M P a)\end{array}$ & $\begin{array}{l}E_{22}^{D} \\
* 10^{-3}(\mathrm{MPa})\end{array}$ & $\begin{array}{l}G_{12}^{D}(\varepsilon) \\
* 10^{-3}(M P a)\end{array}$ & $\begin{array}{l}G_{12}^{D}(\gamma) \\
* 10^{-3}(\mathrm{MPa})\end{array}$ & $\begin{array}{l}\varepsilon_{11}^{p} \\
* 10^{5}\left(\frac{\mathrm{mm}}{\mathrm{mm}}\right)\end{array}$ & $\begin{array}{l}\varepsilon_{22}^{p} \\
* 10^{3}\left(\frac{m m}{m m}\right)\end{array}$ & $\begin{array}{l}\varepsilon_{12}^{p} \\
* 10^{3}\left(\frac{m m}{m m}\right)\end{array}$ & $\begin{array}{l}\gamma_{12}^{p} \\
* 10^{3}\left(\frac{m m}{m m}\right)\end{array}$ \\
\hline-28.244 & 5.572 & 3.919 & 1.959 & 0.000 & 0.000 & 0.000 & 0.000 \\
\hline-25.052 & 4.523 & -3.425 & -1.713 & 0.860 & -0.672 & -0.340 & -0.681 \\
\hline-32.429 & 4.054 & -3.095 & -1.548 & 1.471 & -1.303 & -0.659 & -1.318 \\
\hline-417.031 & 3.581 & -2.781 & -1.391 & 1.720 & -1.891 & -0.954 & -1.908 \\
\hline 71.577 & 3.093 & -2.421 & -1.211 & 0.330 & -2.783 & -1.393 & -2.786 \\
\hline 74.038 & 3.033 & -2.374 & -1.187 & -8.228 & -4.161 & -2.039 & -4.078 \\
\hline
\end{tabular}

Damage in the principal material directions and thermodynamic damage energy release rates were calculated as preciously seen in the $\pm 45^{0}$ section, and tabulated in Table A-0-17:

Table A-0-17: $\pm 67.5^{0}$ Calculated damages and thermodynamic force conjugates

\begin{tabular}{|c|c|c|c|c|c|c|c|}
\hline \multicolumn{4}{|c|}{ Damage } & \multicolumn{4}{c|}{ Thermodynamic force conjugate of damage } \\
\hline $\boldsymbol{D}_{\mathbf{1 1}}$ & $D_{22}$ & $D_{12}\left(\varepsilon_{12}\right)$ & $D_{12}\left(\gamma_{12}\right)$ & $Y_{22}(M P a)$ & $\sqrt{Y_{22}}(\sqrt{M P a})$ & $Y_{12}(M P a)$ & $\sqrt{Y_{12}}(\sqrt{M P a})$ \\
\hline 0.000 & 0.000 & 0.000 & 0.000 & 0.000 & 0.000 & 0.000 & 0.000 \\
\hline 0.113 & 0.188 & 1.874 & 1.874 & 0.152 & 0.390 & 0.057 & 0.238 \\
\hline-0.148 & 0.272 & 1.790 & 1.790 & 0.384 & 0.620 & 0.140 & 0.374 \\
\hline-13.765 & 0.357 & 1.710 & 1.710 & 0.697 & 0.835 & 0.246 & 0.496 \\
\hline 3.534 & 0.445 & 1.618 & 1.618 & 1.272 & 1.128 & 0.441 & 0.664 \\
\hline 3.621 & 0.456 & 1.606 & 1.606 & 1.517 & 1.232 & 0.527 & 0.726 \\
\hline
\end{tabular}

Noting, that the maximum value in the $\sqrt{Y_{22}}$ column is $\sqrt{Y_{22}^{U}}$ :

$$
\max \left(\sqrt{Y_{22}}\right)=\sqrt{Y_{22}^{U}}
$$

The thermodynamic conjugate $Z(\varepsilon)$, transverse-shear coupling constant $b$, and $\underline{\hat{Y}}$ were calculated using Eqs. (4. 41-4. 43):

$$
\begin{gathered}
Z_{12_{i}}\left(\varepsilon^{e}\right)=\frac{1}{2} E_{12}^{0}\left(\operatorname{Run} \gamma_{12}\right)_{i}^{2} \text { and } Z_{22_{i}}\left(\varepsilon^{e}\right)=\frac{1}{2} E_{22}^{0}\left(v_{12}^{0} \text { Run }_{11_{i}}+\text { Run }_{22_{i}}\right)^{2} \\
b_{i}=\frac{\left(\left(D_{12}\left(\gamma_{12}\right) * \sqrt{Y_{12}^{c}}+\sqrt{Y_{12}^{0}}\right)^{2}-Z_{12_{i}}\left(\varepsilon^{e}\right)\right)}{Z_{22_{i}}\left(\varepsilon^{e}\right)} \\
\hat{\underline{Y}}_{i}=\left(\underline{Y 12}_{i}+b \underline{Y 22}_{i}\right)
\end{gathered}
$$


Table A-0-18: $\pm 67.5^{0}$ Thermodynamic conjugates and damage initiation threshold

\begin{tabular}{|c|c|c|c|c|}
\hline $\boldsymbol{Z}_{\mathbf{1 2}}\left(\boldsymbol{\varepsilon}^{\boldsymbol{e}}\right)$ & $\boldsymbol{Z}_{\mathbf{2 2}}\left(\boldsymbol{\varepsilon}^{\boldsymbol{e}}\right)$ & $\boldsymbol{b}$ & $\widehat{\boldsymbol{Y}}(\boldsymbol{M P a})$ & $\sqrt{\widehat{\boldsymbol{Y}}}(\sqrt{\boldsymbol{M P a}})$ \\
\hline 0.000 & 0.000 & 0.000 & 0.000 & 0.000 \\
\hline 0.057 & 0.149 & 108.541 & 5.708 & 2.389 \\
\hline 0.139 & 0.376 & 39.588 & 14.394 & 3.794 \\
\hline 0.245 & 0.694 & 19.783 & 26.118 & 5.111 \\
\hline 0.437 & 1.264 & 9.695 & 47.634 & 6.902 \\
\hline 0.524 & 1.516 & 7.912 & 56.832 & 7.539 \\
\hline
\end{tabular}

The following calculations are related to test 1 - laminate level, and similar calculations have been performed for test 2 and 3:

Points of interest, damaged elastic moduli, plastic strain, damage, thermodynamic force, shear integrity, plastic strain, and power law predictions were calculated as previously seen in the $\pm 45^{0}$ section, and tabulated in tables 25 to 27 :

Table A-0-19: $\pm 67.5^{0}$ Points of interested and their respective rise and run

\begin{tabular}{|c|c|c|c|c|c|c|c|c|}
\hline \multicolumn{3}{|c|}{ UPPER limit of cycle } & \multicolumn{6}{|c|}{ LOWER limit of cycle } \\
\hline \multirow[t]{2}{*}{$\sigma_{L}^{U}(M P a)$} & \multirow{2}{*}{$\begin{array}{c}\varepsilon_{L}^{U} * 10^{2} \\
\left(\frac{m m}{m m}\right)\end{array}$} & \multirow{2}{*}{$\begin{array}{c}\varepsilon_{T}^{U} * 10^{3} \\
\left(\frac{m m}{m m}\right)\end{array}$} & \multirow[t]{2}{*}{$\sigma_{L}^{L}(M P a)$} & \multirow{2}{*}{$\begin{array}{c}\varepsilon_{L}^{L} * 10^{2} \\
\left(\frac{m m}{m m}\right)\end{array}$} & \multirow{2}{*}{$\begin{array}{c}\varepsilon_{T}^{L} * 10^{4} \\
\left(\frac{m m}{m m}\right)\end{array}$} & \multicolumn{2}{|l|}{ Longitudinal } & \multirow{2}{*}{$\begin{array}{l}\text { Transverse } \\
\text { Run } \\
* 10^{3}\left(\frac{\mathrm{mm}}{\mathrm{mm}}\right)\end{array}$} \\
\hline & & & & & & Rise $_{L}(M P a)$ & $\begin{array}{l}\text { Run }_{L} \\
* 10^{2}\left(\frac{m m}{m m}\right)\end{array}$ & \\
\hline 0.000 & 0.000 & 0.000 & 0.000 & -50.250 & 6.190 & 0.000 & 0.000 & 0.000 \\
\hline-38.482 & -0.978 & 1.924 & 0.000 & -0.081 & 1.496 & -38.482 & -0.897 & 1.774 \\
\hline-54.778 & -1.580 & 2.987 & -0.211 & -0.163 & 2.981 & -54.568 & -1.417 & 2.689 \\
\hline-65.180 & -2.139 & 3.714 & -0.108 & -0.232 & 4.179 & -65.072 & -1.907 & 3.296 \\
\hline-76.035 & -2.912 & 4.838 & -0.400 & -0.350 & 6.027 & -75.635 & -2.563 & 4.235 \\
\hline-81.443 & -3.315 & 5.422 & -0.227 & -0.508 & 7.754 & -81.216 & -2.806 & 4.647 \\
\hline-86.214 & -3.726 & 6.470 & & & & & & \\
\hline
\end{tabular}

Table A-0-20: $\pm 67.5^{0}$ Damaged elastic moduli, plastic strain, damage, and energy release rates

\begin{tabular}{|c|c|c|c|c|c|c|c|}
\hline \multicolumn{2}{|c|}{ Damaged Elastic Moduli } & \multicolumn{3}{|c|}{ Plastic Strain (apparent) } & \multirow{2}{*}{$\begin{array}{c}\text { Damage } \\
\qquad D_{T}\end{array}$} & \multicolumn{2}{|c|}{$\begin{array}{c}\text { Thermodynamic damage energy } \\
\text { release rate }\end{array}$} \\
\hline$E_{L}^{D}(M P a)$ & $E_{T}^{D}(M P a)$ & $\begin{array}{l}\varepsilon_{L}^{p} \\
* 10^{3}\left(\frac{m m}{m m}\right)\end{array}$ & $\begin{array}{l}\varepsilon_{T}^{p} \\
* 10^{4}\left(\frac{m m}{m m}\right)\end{array}$ & $D_{L}$ & & $Y_{L}(\sigma)(M P a)$ & $\sqrt{Y_{L}(\sigma)}(\sqrt{M P a})$ \\
\hline 5173.200 & 25553.000 & 0.000 & 0.000 & 0.000 & 0.000 & 0.000 & 0.000 \\
\hline 4291.497 & 21691.511 & -0.813 & 1.496 & 0.170 & 0.151 & 0.208 & 0.456 \\
\hline 3851.988 & 20296.255 & -1.576 & 3.084 & 0.255 & 0.206 & 0.523 & 0.723 \\
\hline 3412.273 & 19743.793 & -2.286 & 4.234 & 0.340 & 0.227 & 0.944 & 0.971 \\
\hline 2951.267 & 17859.408 & -3.360 & 6.251 & 0.430 & 0.301 & 1.717 & 1.310 \\
\hline 2893.939 & 17477.710 & -5.005 & 7.883 & 0.441 & 0.316 & 2.049 & 1.431 \\
\hline
\end{tabular}


Table A-0-21: $\pm 67.5^{0}$ Shear integrity, plastic strain, and yield function points

\begin{tabular}{|c|c|c|c|c|c|}
\hline $\begin{array}{c}\text { Shear } \\
\text { integrity }\end{array}$ & $\begin{array}{c}\text { Plastic Strain } \\
\text { Increment }\end{array}$ & $\begin{array}{c}\text { Accumulated Plastic } \\
\text { Strain }\end{array}$ & $\begin{array}{c}\text { Experimental points for } \\
\text { yield function }\end{array}$ & $\begin{array}{c}\text { Power law } \\
\text { prediction for R }\end{array}$ & Error \\
\hline $\boldsymbol{\Omega}_{\boldsymbol{i}}$ & $\Delta p_{\boldsymbol{i}} * 10^{4}$ & $p_{i} * 10^{4}$ & $f_{i}(\mathrm{MPa})$ & 0.000 & 0 \\
\hline 1.000 & 0.000 & 0.000 & 0.000 & 49.069 & 0.0033 \\
\hline 0.830 & 7.439 & 7.439 & 46.388 & 76.590 & 0.0017 \\
\hline 0.745 & 6.006 & 13.445 & 73.567 & 97.099 & 0.0003 \\
\hline 0.660 & 4.986 & 18.431 & 98.816 & 122.242 & 0.0069 \\
\hline 0.570 & 6.601 & 25.032 & 133.279 & 155.021 & 0.0042 \\
\hline 0.559 & 9.297 & 34.329 & 145.588 & & $R_{i}(\mathrm{MPa})$ \\
\hline
\end{tabular}




\section{Appendix B: Sample MMDM Code}

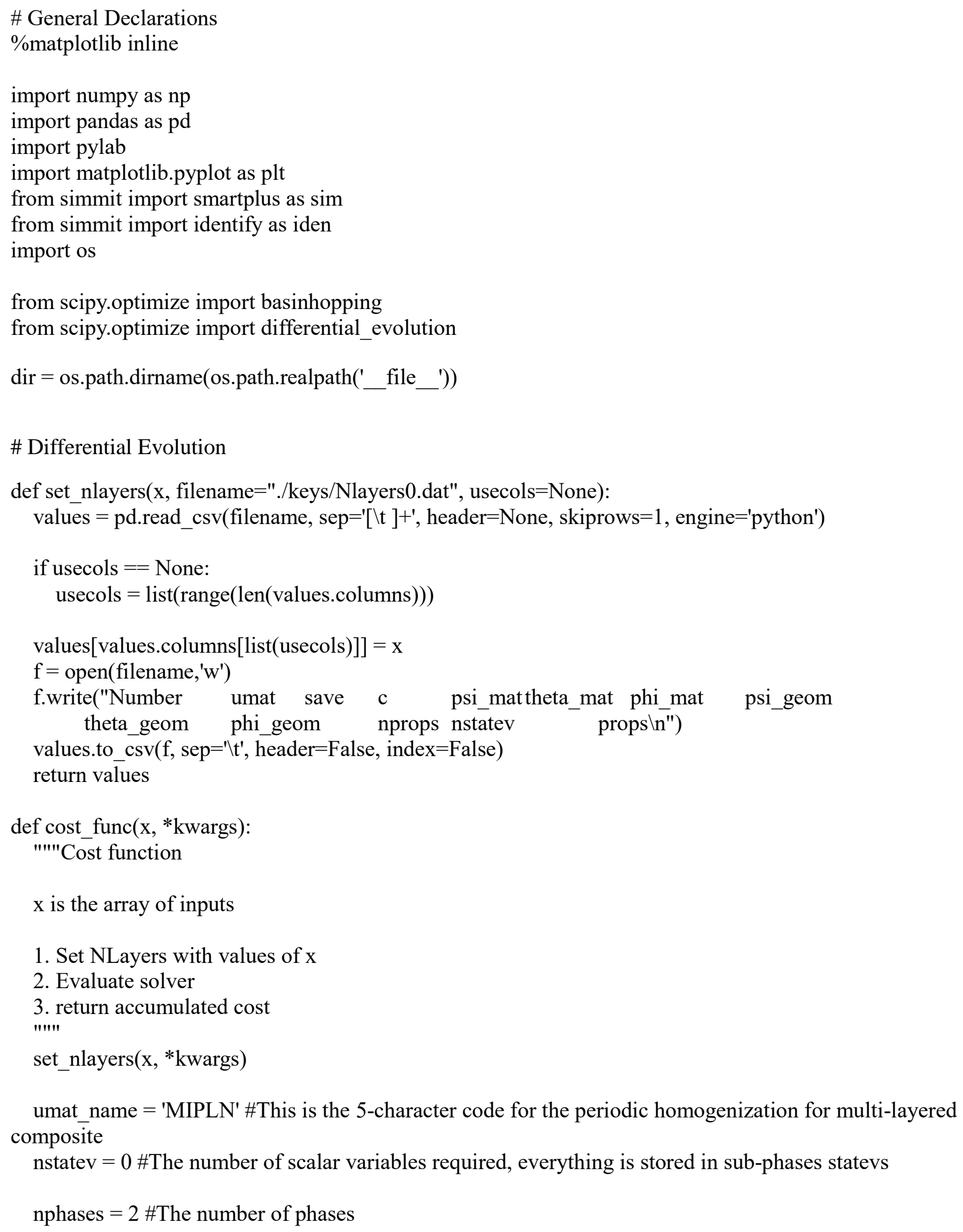


num_file $=0$ \#The num of the file that contains the subphases

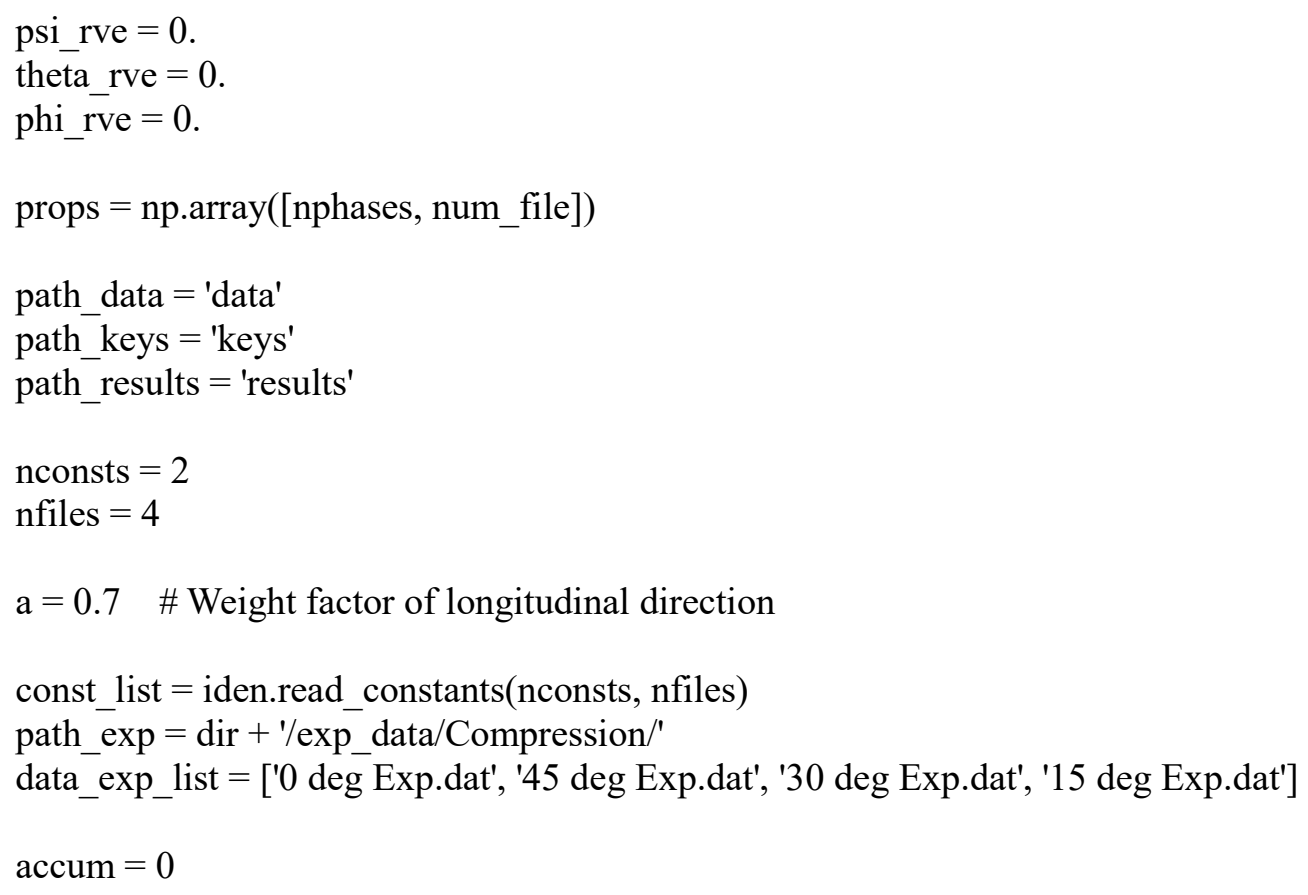

outputfile $=$ path_results + '/' + 'Comp_simul' + str(n_simul +1$)+$ '_global-0.txt'; e11, e22, e33, e12, e13, e23, s11, s22, s33, s12, s13, s23 = np.loadtxt(outputfile, usecols $=(8,9,10,11,12,13,14,15,16,17,18,19)$, unpack $=$ True $)$

pred_11 $=-\mathrm{e} 11$

pred_22 $=-\mathrm{e} 22$

$\operatorname{print}(\operatorname{len}(\mathrm{e} 11))$

exp_file $=$ path_exp+data_exp_list[n_simul]

e11,e22,s11 = np.loadtxt(exp_file, usecols $=(2,3,4)$, unpack=True $)$

exp_11 $=-$ e11

exp_22 = -e22

$\operatorname{print}(\operatorname{len}(\mathrm{e} 11))$ 


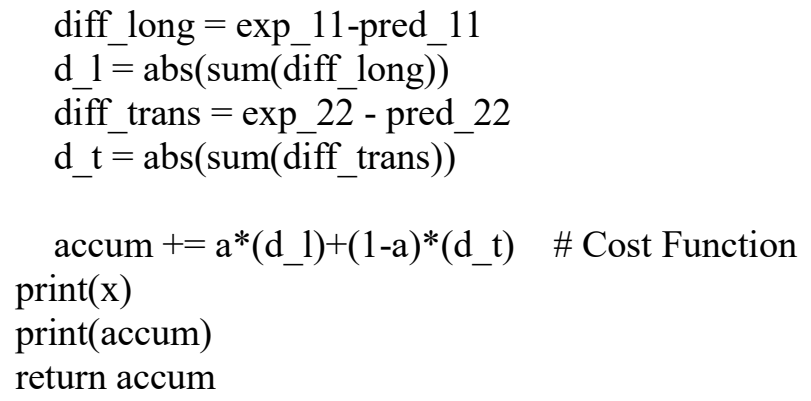

$\mathrm{xmin}=[24500,5660,0.077,0.36,1700,0.10,0.40,0.22,266]$

$\mathrm{xmax}=[24560,5720,0.090,0.40,2100,0.30,25.0,0.33,404]$

bounds $=[($ low, high $)$ for low, high in $\operatorname{zip}(\mathrm{xmin}, \mathrm{xmax})]$

best_ $\mathrm{x}=$ differential_evolution(cost_func, bounds, args=("./keys/Nlayers0.dat", usecols), strategy $=$ 'best 1 bin', maxiter $=100000$, popsize $=5$, mutation $=(0.81,0.99)$, recombination $=0.76$, polish $=$ True $)$ 


\section{References}

1. T. Kraus, M. Kühnel (2014). Global carbon fibre market remains on upward trend. www.reinforcedplastics.com, pp. 38-45

2. Statista (2017), Forecast demand global boron market glass fiber 2012-2023 https://www.statista.com/statistics/449795/worldwide-glass-fiber-latent-demandforboron-prediction/

3. Transparency Market Research (2017). Glass fiber market - global industry analysis, size, share, growth, trends, and forecast 2016 - 2024.

<http://www.transparencymarketresearch.com/glass-fibers-market.html>

4. Oregon State University, Environmental Health and Safety (2009). When Is Asbestos Dangerous?. ehs.oregonstate.edu/asb-when

5. D. B. Dittenber, H. V. S. GangaRao (2012). Critical review of recent publications on use of natural composites in infrastructure. Composites Part A 43 (8): 1419-1429

6. D., Yielin, Y. Tian (2015). Assessing the environmental impact of flax fibre reinforced polymer composite from a consequential life cycle assessment perspective Sustainability, 7: $11462-11483$

7. J. A. Zsriros, (2010). Natural Fibers and Fiberglass: A Technical and Economic Comparison. Thesis. Brigham Young University - School of Technology, Brigham Young U

8. A. Le Duigou, Davies P., Baley C (2011). Environmental impact analysis of the production of flax fibres to be used as composite material reinforcement. Journal of Biobased Materials and Bioenergy 5: 1-13

9. K. L. Pickering, M. G. Aruan Efendy, T. Tm. Le (2016). A review of recent developments in natural fibre composites and their mechanical performance. Composites Part A 83: 98 $-112$

10. R. A. Khan, M.A. Khan, H.U. Zaman, S. Pervin, N. Khan (2009). Comparative studies of mechanical and interfacial properties between jute and e-glass fiber-reinforced Polypropylene Composites. Journal of Reinforced Plastics and Composites. 29(7): 1078 1088. 
11. STEMERGY (2017). Renewable Fiber Technology.Products: Stemergy Renewable Bio Fiber, <http://www.stemergy.com/products/flaxfibre/flaxfibercomposites/>

12. CARBIO (2017). Carbon-Flax Hybrid structures for automotive applications. $<$ http://carbioproject.com/>

13. "Faurecia (2015). Flaxpreg TM Wins JEC Europe 2015 Innovation Award in the SemiProducts Category. <http://www.faurecia.com/en/faurecia-flaxpregtm-wins-jeceurope-

2015-innovation-award-semi-products-category>

14. Composites Evolution (2015). Flax used to improve impact performance for bamboo bike. http://compositesevolution.com/news/biotex-flax-used-to-improve-impactperformancefor-bamboo-bike/

15. DUDYE (2010), JEC Innovation Awards. Announcing the 11 Winners of This Year's JEC Innovation Awards Programme. <http://dudye.com/announcing-the-11-winners-ofthisyears-jec-innovation-awards-programme>.

16. Composites Evolution (2014). Blades for rooftop wind turbine manufactured from composites evolution's Biotex Flax. <http://compositesevolution.com/news/bladesforrooftop-wind-turbine-manufactured-from-composites-evolutions-biotex-flax/>.

17. North Dakota State University (2012). History of Flax. <https://www.ag.ndsu.edu/ccv/flax/history-and-uses-1>

18. S.S. Muthu, (2014). Roadmap to sustainable textiles and clothing: environmental and social aspects of textiles and clothing supply chain. Textile Science and Clothing Technology, Springer, 2014. 3-23.

19. I.K. Mazharul (2011). Flax Fiber. Textile Learner. http://textilelearner.blogspot.ca/2011/08/flax-fiber-properties-of-flax-fiber_8109.html.

20. H. L. Bos, A. M. Donald (1999). In situ SEM study of the deformation of elementary flax fibres. Journal of Materials Science, 34(13): 3029-3034.

21. H. L Bos., J. Mussing, M. J.A. Van Den Oever (2016). Mechanical Properties of Shortflaxfibre Reinforced Compounds. Composites Part A: Applied Science and Manufacturing 37(10): 1591-604

22. C. Baley (2002), Analysis of the flax fibres tensile behaviour and analysis of the tensile stiffness increase, Compos Part A 33 (7): 939-948 
23. B., Lamy, C. Baley (2000). Stiffness prediction of flax fibers-epoxy composite materials. Journal of Materials Science Letters, 19(11): 979-980.

24. G. Romhány, J. Karger-Kocsis, T. Czigány (2003). Tensile fracture and failure behavior of technical flax fibers. Journal of Applied Polymer Science, 90(13): 3638-3645.

25. P. S. Mukherjee, K. G. Satyanarayana (1986). An empirical evaluation of structureproperty relationships in natural fibres and their fracture behaviour. J Mater Sci 21 (12): 4162-4168

26. H. L. Bos, M. J. A. van den Oever, O. C. J. J. Peters (2002). Tensile and compressive properties of flax fibres for natural fibre reinforced composites. Journal of Materials Science, 37(8): 1683-1692.

27. Z. Mahboob, I.E. Sawi, R. Zdero, Z. Fawaz, H. Bougherara (2017). Tensile and compressive damaged response in Flax fibre reinforced epoxy composites. Composites: Part A 92: 118-133

28. P.O. Hagstrand, K. Oksman (2001). Mechanical properties and morphology of flax fiber reinforced melamine-formaldehyde composites. Polymer Composites, 22(4): 568-578

29. Z. Mahboob, Y. Chemisky, F. Meraghni, H. Bougherara (2017). Mesoscale modelling of tensile response and damage evolution in flax fibre reinforced laminates. Composites Part B 119: 168-183

30. Herakovich, C.T. (1989). Damage Mechanics. Mechanics of Fibrous Composites. New York: Wiley, 322-60

31. A. Corigliano (2003). Damage and fracture mechanics techniques for composite structures, in: Numerical and Computational Methods, vol. 3 of Comprehensive Structural Integrity, Elsevier Pergamon, Amsterdam; San Diego CA. 459-539

32. Overview Brittle and Ductile Materials. University of Liege. http://www.ltascm3.ulg.ac.be/FractureMechanics/overview_P3.html\#PictureI49

33. J. Lemaitre, J.L. Chaboche (1994). Mechanics of Solid Materials. Cambridge: Cambridge

34. P. Ladeveze, E. Le Dantec (1992). Damage modelling of the elementary ply for laminated composites. Composite Science and Technology 43.3 (1992): 257-167

35. ASTM Committee, 2017, ASTM D6641/D6641M-16e1 Standard Test Method for Compressive Properties of Polymer Matrix Composite Materials Using a Combined Loading Compression (CLC) Test Fixture, ASTM International 
36. ASTM Committee, 2017, ASTM D695 - 15 Standard Test Method for Compressive Properties of Rigid Plastics, ASTM International

37. ASTM D30 Committee, 2011, ASTM D3171 Test Methods for Constituent Content of Composite Materials, ASTM International

38. S. Phillips, J. Baets, L. Lessard, P. Hubert, I Verpoest (2013). Characterization of flax/epoxy prepregs before and after cure. Journal of Reinforced Plastics and Composites, 32(11): 777-785

39. I. El Sawi, H. Bougherara, R. Zitoune, Z Fawaz (2014). Influence of the Manufacturing Process on the Mechanical Properties of Flax/Epoxy Composites. Journal of Biobased Materials and Bioenergy, 8(1): 69-76

40. ASTM E2015. Standard Guide for Preparation of Plastics and Polymeric Specimens for Microstructural Examination, ASTM International

41. Knipprath, C. (2010) Mechanical Performance of Binder Yarn Composites. Diss. Cranfield U

42. ZernoExport. "Flax Seeds." ZernoExport. N.p., n.d. Web. 14 July 2017 <http://www.zernoexport.com/en/catalog/catalog3/lenmasl>

43. Optimization and Root Finding (scipy.optimize. https://docs.scipy.org/doc/scipy0.18.1/reference/optimize.html

44. Smart Materials Algorithms and Research Tools. $<$ http://www.lem3.fr/chemisky/smartplus/>

45. A. Bensoussan, J.-L. Lions, G. Papnicolaou. (2011). Asymptotic Analysis for Periodic Structures, Vol. 374, American Mathenmatical Society Chelsea Publishing.

46. G. Chatzigeorgiou, N. Charalambakis, Y. Chemisky, F. Meraghni, Periodic homogenization for fully coupled thermomechanical modeling of dissipative generalized standard materials, Int J Plasticity 81 (2016) 18-39

47. S. Amijima, T. Adachi. (1979). Nonlinear stress-strain response of laminated composites. Journal of composite materials. 13(3): 206-218 